4613-20-R

Report of BAMIRAC

\title{
METHODS OF RADIOMETRIC CALIBRATION
}

FRED E. NICODEMUS

GEORGE J. ZISSIS

October 1962

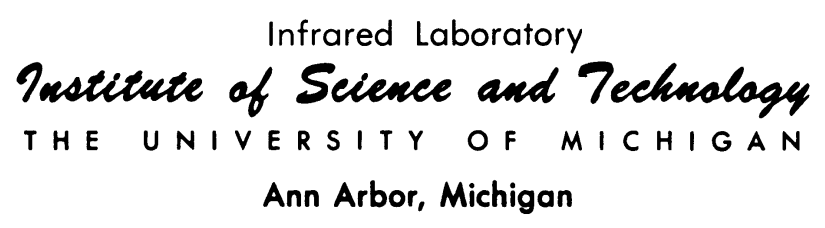




\section{NOTICES}

Sponsorship. The work reported herein was conducted by the Institute of Science and Technology for the Advanced Research Projects Agency, Department of Defense, under Contract SD-91 (ARPA Order 236) as a part of Project DEFENDER (research on defense against ballistic missiles). Contracts and grants to The University of Michigan for the support of sponsored research by the Institute of Science and Technology are administered through the Office of the Vice-President for Research.

Acknowledgments. The authors gratefully acknowledge the many informative and inspiring discussions with: E. Hall, A. LaRocca, W. Wolfe, A. G. DeBell, C. E. Dunning, H. L. Sachs, J. F. Coughlin, R. Sexauer, F. W. Cramer, P. R. Gast, S. N. Ghosh, A. R. Karoli, A. R. Dennett, E. W. Manring, W. W. Talbert, W. C. Schupp, and M. Kienitz. Portions of this report have stemmed from material prepared informally by F. Nicodemus for use by the U. S. Army Signal Missile Support Agency at the White Sands Missile Range in the calibration of a rapid-scan spectrometer. Other portions are an outgrowth of earlier work performed by A. LaRocca and G. Zissis on Project MICHIGAN, under Contract DA-36-039 SC-52654, between The University of Michigan's Institute of Science and Technology and the U. S. Army Signal Corps. The release by Sylvania Electronic Defense Laboratories of F. Nicodemus for the time needed to prepare this report is gratefully acknowledged.

Final Disposition. After this document has served its purpose, it may be destroyed. Please do not return it to the Institute of Science and Technology. 


\section{PREFACE}

The needs of military programs such as ARPA's Project DEFENDER have led to a host of infrared target measurement programs. The targets range from ballistic missile nose cones during their re-entry into the atmosphere to ships at sea and vehicles on roads. The measurements are made from aircraft, rockets, ships, and other platforms, with instruments varying from simple pinhole radiometers to sophisticated spatial-scanning interferometer-spectrometers. Often the wavelengths observed cover a range from the ultraviolet to microwave. The results of these measurement programs are useful only if several conditions are met. One which is absolutely necessary is that the instruments used must be adequately calibrated.

Although this requirement is simple to state, in practice the difficulties are great and the methods usually complex. In view of the critical importance of this process called "calibration," a tutorial report has been prepared by the Institute of Science and Technology, The University of Michigan, under Contract SD-91, which is a part of ARPA's Project DEFENDER. The report is being issued in this preliminary form to a small group of scientists for immediate use in the hope that a series of comments and contributions will be generated. After allowing time to receive these reactions, the authors will review the report critically and reissue it to a much larger audience. Comments are solicited as to the subject matter and the desirable degree of coverage for each topic. Little is said in this report, for example, about calibration sources. Is this a subject deserving a much deeper and more exhaustive treatment? Should a-c radiometry and calibration methods be separately treated? Only by this process can a useful document be made in view of the pitfalls in performing calibrations and, even more, the perils in writing about calibrations.

Contract SD-91 supports the Ballistic Missile Radiation Analysis Center (BAMIRAC), a facility which functions as a research and technical information center on phenomenology, theory, and that technology pertaining to the fundamental phenomena associated with ballistic missiles and space vehicles which may be significant in the formulation of defense measures against missile and space-vehicle systems. BAMIRAC collects and processes information on electromagnetic and acoustic radiation emanating from or caused by ICBM's or IRBM's during their trajectory from launch to impact. The information includes field measurements, laboratory studies, and theoretical studies, and is available at the BAMIRAC reference library for use by representatives from all organizations presenting a properly authorized request. BAMIRAC conducts analyses in which experimental and theoretical results are evaluated and examined for correlations. Some theoretical and experimental investigations are carried out, and the results are combined with the technical information obtained from outside sources. In its capacity as a technical information center, BAMIRAC disseminates information by 
means of abstracts, indexes, bibliographies, technical reports and journal articles, technical meetings, and the Proceedings of the Anti-Missile Research Advisory Council.

BAMIRAC is under the technical direction of the Infrared Laboratory. It draws also, however, upon the capabilities of the Computation Department of the Institute of Science and Technology, and upon those of the Aircraft Propulsion Laboratory of the Department of Aeronautical and Astronautical Engineering and the Radiation Laboratory of the Department of Electrical Engineering, both of which are organized within The University of Michigan's College of Engineering. 


\section{CONTENTS}

Notices. . . . . . . . . . . . . . . . . . . . . . . . . . ii

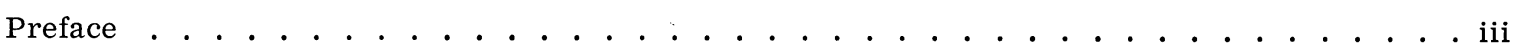

List of Figures . . . . . . . . . . . . . . . . . . . . . . . . vi

Abstract .................................... 1

1. Introduction . . . . . . . . . . . . . . . . . . . . . . . . . 1

2. Calibration of a Basic Radiometer: Ideal Case . . . . . . . . . . . . . . . . . 3

2.1. The Instrument

2.2. Instrument Responsivity 15

2.3. Responsivity Calibration 6

2.3.1. Distant Small-Source Calibration 6

$\begin{array}{ll}\text { 2.3.2. Distant Extended-Source Calibration } & 7\end{array}$

2.3.3. Near Extended-Source Calibration $\quad 8$

2.3.4. Near Small-Source (Jones Method) Calibration 8

2.3.5. Summary of Source Size and Placement Configuration $\quad 10$

$\begin{array}{ll}\text { 2.3.6. Use of a Collimator } & 10\end{array}$

2.3.7. Reference Radiation $\quad 10$

3. Calibration of a Basic Radiometer: Non-Ideal Case . . . . . . . . . . . . . . . . 11

3.1. The Instrument 13

3.2. Instrument Responsivity 13

3.3. Spectral Responsivity $r$

$\begin{array}{ll}3.3 .1 \text {. Normalization } & 15\end{array}$

3.3.2. Spectral Responsivity Calibration $\quad 20$

3.4. Spatial Responsivity $\quad 22$

3.4.1. Directional Responsivity-Use of a Field Lens 22

3.4.2. Mapping the Field 23

3.4.3. Computation of Effective Field Solid Angle 26

3.5. Temporal Responsivity 29

$\begin{array}{ll}\text { 3.5.1. Temporal Responsivity Calibration } & 30\end{array}$

3.6. Referral of Measured Quantities to the Source $\quad 30$

3.6.1. Background Sources $\quad 31$

3.6.2. Atmospheric Attenuation-Apparent Source Radiation 32

3.6.3. Apparent Source Temperature vs. Apparent Source Radiation $\quad 34$

3.7. Linearity

3.7.1. Linearity Calibration $\quad 35$

3.8. Detector Temperature $\quad 37$

4. Calibration of a Chopper Radiometer . . . . . . . . . . . . . . . . . . . 38

4.1. Chopper as Source of Reference Radiation 38

$\begin{array}{ll}\text { 4.1.1. Location of Chopper } & 39\end{array}$

4.2. Waveform Problems $\quad 40$

5. Calibration of Spectroradiometers . . . . . . . . . . . . . . . . . . 40

5.1. Prism and Grating Instruments 42

5.2. Absolute vs. Relative Calibration of Spectroradiometers 49

$\begin{array}{ll}\text { 5.3. Interferometers } & 49\end{array}$

6. Calibration Sources . . . . . . . . . . . . . . . . . . . . . 52

7. Summary . . . . . . . . . . . . . . . . . . . . . . . 52

References.................................54

Bibliography ................................. 56

Distribution List . . . . . . . . . . . . . . . . . . . . . . . . . 62 


\section{FIGURES}

1. A Simple Form of Basic Radiometer. . . . . . . . . . . . . . . . . . . . . . . . 4

2. Distant Small-Source Calibration . . . . . . . . . . . . . . . . . . . 6

3. Distant Extended-Source Calibration. . . . . . . . . . . . . . . . . . . 7

4. Near Extended-Source Calibration. . . . . . . . . . . . . . . . . . 8

5. Near Small-Source (Jones Method) Calibration . . . . . . . . . . . . . . . . . 9

6. Method of Designating Direction of Ray. . . . . . . . . . . . . . . . . . . . 12

7. Spectral Power Responsivity and Spectral Output . . . . . . . . . . . . . . . . 16

8. Geometry for Mapping the Field with a Distant Small Source . . . . . . . . . . . . . . . 24

9. Geometry for Mapping the Field with a Collimator . . . . . . . . . . . . . . . . . . 25

10. Solid Angle of Circular Field Stop . . . . . . . . . . . . . . . . . . . . . . . 27

11. A Simple Form of Spectroradiometer . . . . . . . . . . . . . . . . . . . . . . . . . 42

12. Triangular Spectral Slit Width . . . . . . . . . . . . . . . . . . . . . 45

13. Trapezoidal Spectral Slit Width-Unequal Slits . . . . . . . . . . . . . . . . . . 47

14. Michelson Interferometer. . . . . . . . . . . . . . . . . . . . . . 51 


\title{
METHODS OF RADIOMETRIC CALIBRATION
}

\begin{abstract}
This report presents a tutorial and general treatment of the calibration of optical instruments which are to be used to measure electromagnetic radiation from sources of interest to the military services. The infrared spectral region is emphasized although the ultraviolet and visible wavelengths are also treated. The report is being issued in a preliminary form to a limited group of scientists in an attempt to stimulate comment and criticism.
\end{abstract}

1

INTRODUCTION

This report deals with the calibration of optical instrumentation used to measure characteristics of electromagnetic radiation in order to obtain information about the source of the radiation. Thus it will be concerned with radiometers responding primarily in the ultraviolet, visible, and infrared regions of the electromagnetic spectrum. The term radiometer is used here in its broadest sense, so that it includes specialized devices which can be used to measure a particular characteristic of the radiation field, such as spectrometers for measuring the spectral distribution of radiation, or scanners for measuring the spatial (directional) distribution.

A discussion of calibration methods and problems can be helpful as a guide in planning and conducting instrument calibration. It can point out pitfalls to be avoided, or possible sources of error, and possible techniques for eliminating or minimizing their effects. However, the exact procedure to be followed in calibrating a particular instrument depends upon the details of that instrument and the way in which it is used. In addition, it cannot be too strongly emphasized that the quality of calibration, and hence the quality of subsequent measurements, depends primarily on the familiarity of the operator with all of the details of his instrument and its operation, and on his dedication to thoroughness and accuracy. Intimate knowledge of the particular instrumentation, gained by operation over a period of time sufficiently long to encounter a variety of circumstances, and a passion for precision are essential for any reliable calibration.

Measurement is the process by which a quantitative comparison is established between two or more values of a physical observable, such as length, temperature, or spectral radiance. A report of a measurement is of limited usefulness, however, unless at least one of the observed quantities is available, for comparison or reference, to those receiving the report. Absolute calibration is the process by which measurements are referred to standard values, such as the primary standards maintained by the U. S. National Bureau of Standards. These primary standards are widely available, at least through comparisons or calibra- 
tions with intermediate or secondary reference standards.

The objective of a calibration is to make measurement results independent of the particular measuring instrument or measuring technique employed. In other words, it is desired that when the same magnitude of a physical quantity is measured by different instruments at different times and/or places, and possibly by different methods, the resulting values should always be the same. However, because we can never know exhaustively all of the existing conditions and understand completely all of the pertinent physical laws governing the effects of those conditions in any actual situation, there is always the possibility that we may be overlooking some significant parameter and its effect on a measurement result. For this reason it is a good general rule to carry out calibration under conditions which reproduce, as completely as possible, the situation which exists when measurements based on that calibration are made.

The precision of a measurement is specified by the amount of difference or disagreement to be expected between different measurements or determinations of the same magnitude. It is a measure of the reproducibility or consistency of measurement results within a particular set (e.g., all measurements with a certain instrument). The accuracy of a measurement is specified by the difference between the measured value and the true or correct value of the quantity being measured. Accuracy refers only to absolute measurements, while precision refers to relative quantities and is concerned primarily with reproducibility within the set of measurements and not with their referral to an absolute standard. (For a more complete discussion of this topic see NBS Monograph 41 [ 1].) Of course, neither precision nor accuracy can be determined exactly, but they can be estimated by an error analysis using statistical methods. Estimates should be made and included wherever possible in reporting measurement results. In fact, they should be considered as an essential part of any statement of a measured value. Note that, since accuracy involves reference to absolute standards, any estimate of accuracy necessarily includes a statement regarding the calibration procedure followed, identifying the source of standardization or reference which has been employed.

In strong contrast to many areas of physical measurement, such as the exact measurement of time, where precision of about one part in $10^{9}$ has been attained, the usual precision of the better radiometric measurements is about one part in a hundred, and many measurements are reported for which the error is $20 \%$ or $30 \%$, or even more. While there are basic limitations involved, many of which will be discussed in detail in this paper, the authors believe that much can be done to improve the general quality of radiometric measurements of optical radiation and the reporting of measurement results; measurement quality can be raised substantially if workers become more aware of the sources of error and the precautions which can be taken to avoid them or to reduce their effects.

There are a number of useful references which are recommended for careful study by anyone planning or carrying out a specific calibration of radiometric instrumentation [ 1 through 9]. In particular, the January 1962 report of the Working Group on Infrared Backgrounds [ 9], referred to henceforth as the WGIRB 
Report, has received wide distribution, and it will be assumed that copies are available to readers of this report. Except for the most essential material, we will avoid repeating here things already adequately covered in the WGIRB Report. Reference will be made to particular sections of that report where appropriate. Specifically, the definitions, units, and symbols used in this paper will be those defined in the WGIRB Report. Essentially the same concepts and nomenclature are presented in References 2, 3, 4, 5, and 10, although in some instances the treatment is not as detailed. It is urged that the recommendations in References 1 through 5 be considered carefully, and that they be followed wherever possible. Where departures are necessary we urge that they be explicitly reported and related to the recommended standard practices in such a way as to facilitate intercomparison with measurement reports which do follow the standards. These remarks apply to all radiometric measurements of sources, and not just to the calibration measurements by which such measurements are standardized.

2

\section{CALIBRATION OF A BASIC RADIOMETER: IDEAL CASE}

In this section we will study the fundamental calibration configurations that are used for calibration of nearly every radiometric instrument. This will be done by examining the calibration of a basic radiometer (i.e., one which has only the components which are common to every radiometric instrument). Also, to simplify the discussion still further, we will consider only an ideal case; that is, one in which the instrument functions perfectly and where simplifying assumptions are made to avoid treatment of perturbations due to conditions which cannot be ignored in any real situation. We will defer for later discussion the important problems which arise because of (1) perturbing conditions such as background radiation and atmospheric attenuation, (2) imperfections in actual instruments and in their operation for calibration measurements, and (3) the introduction of additional components and functions in more specialized instruments.

\subsection{THE INSTRUMENT}

Three essential components are present in some form in every radiometer or radiometric instrument (see Figure 1):

(a) A detector element which transduces changes in incident electromagnetic radiation into variations of an easily measured property such as conductance

(b) An optical system including the sensitive surface of the detector which determines the combination of receiving aperture and angular field of view through which radiation is collected, thus delineating the amount of radiation to which the instrument responds 
(c) An amplifier and output indicator, usually electronic, to transform the output of the detector element into the desired form of presentation [9]

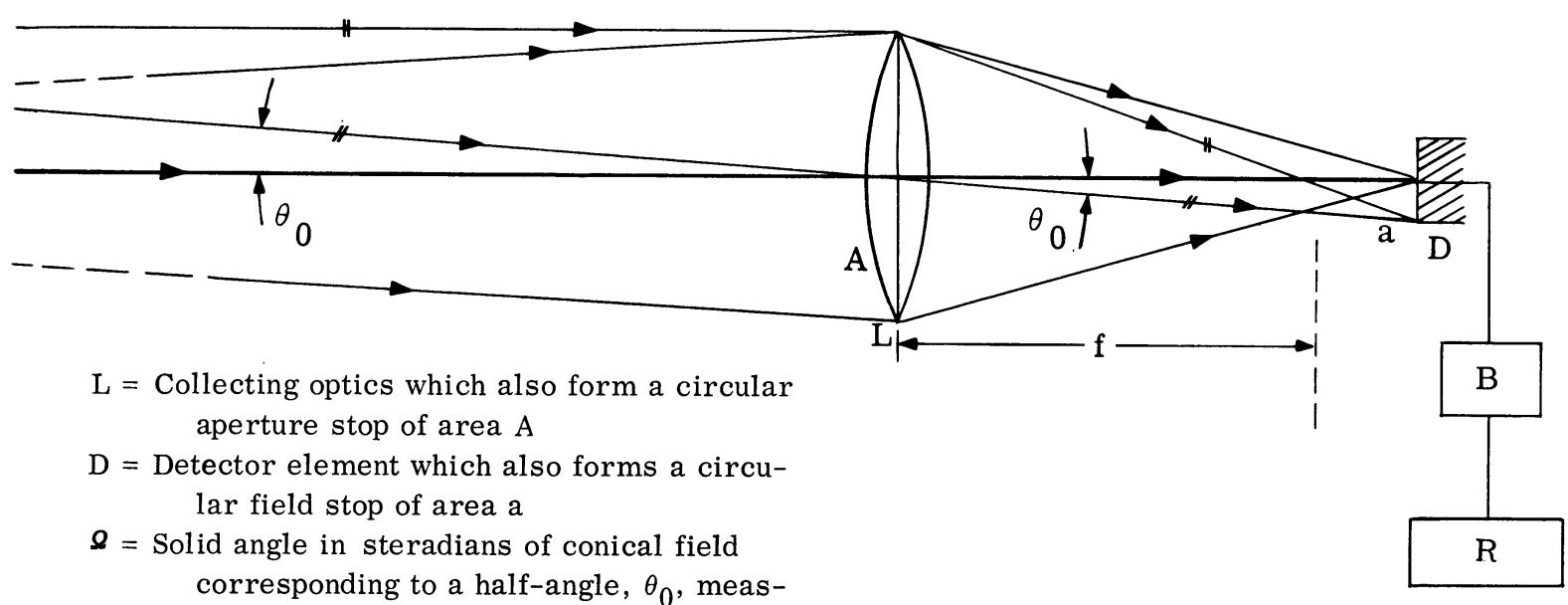
corresponding to a hat ured in radians $\left(\Omega=2 \pi\left(1-\cos \theta_{0}\right) \approx \pi \theta_{0}^{2} \approx \mathrm{a} / \mathrm{f}^{2}\right)$ (see Section 4.4.3)

$\mathrm{B}=$ Amplifier and electronic circuits

$\mathrm{f}=$ Focal length of radiometer

$\mathrm{R}=$ Recorder for output presentation

\section{FIGURE 1. A SIMPLE FORM OF BASIC RADIOMETER}

Because this is to be an ideal case, we will assume also that these components have the following convenient properties (which are only approximated by actual instruments).

(a) The optical system consists of an ideal thin lens which, together with the detector acting as the field stop, sharply defines the circular aperture and angular field of the instrument. Every point of the object (source) is perfectly imaged at the circular field stop (detector surface) regardless of the wavelength of radiation incident on the lens and within the solid angle of the field of view.

(b) The directional (spatial) response is uniform over the field solid angle. Thus, all parts of the optics are perfectly transparent to all rays within the field and the detector is uniformly sensitive across its surface.

(c) The spectral response is constant over a sharply defined wavelength band, $\lambda_{1} \leq \lambda \leq \lambda_{2}$, and is zero outside that band. Thus, the optics are uniformly perfectly transparent, and the detector is uniformly sensitive to all wavelengths within the band. It will be assumed throughout the discussion of this ideal case, except where otherwise stated, that all of the radiometric quantities (e.g., P, J, H, and N) refer only to radiation in this band $\lambda_{1} \leq \lambda \leq \lambda_{2}$.

(d) The detector, amplifier, and output indicator are all linear, so that the output is directly proportional to the radiant power reaching the detector from the field. 
(e) The overall system, including detector, amplifier, and output indicator, responds uniformly to all temporal frequencies present in the fluctuations in the level of the radiation being measured.

\subsection{INSTRUMENT RESPONSIVITY}

We leave our limited ideal situation temporarily for some considerations which apply to all calibration measurements. Radiometers must be calibrated for the measurement of either the amount of radiant power, $P$, received through the entrance aperture and field of the instrument, or the irradiance, $H$, at the aperture received through the field of the instrument, or the radiance, $\mathrm{N}$, of an extended source which covers the aperture and fills the field. Radiometers are also used to measure the way in which any of these radiometric quantities varies with wavelength, direction, or time; but the effects oî these important parameters will be considered in later sections.

The reference location for these incident radiometric quantities is the entrance aperture of the instrument. This location is dictated by our objective of calibration, which makes measurements independent of the particular instrument used. Later, we must also consider how other factors, such as atmospheric transmission and background radiation, affect our attempts to relate measured values of the field quantities at this reference location to radiometric properties of the source (see Section 2.3.4 of Reference 9 for a more detailed discussion of radiometric quantities as field concepts).

The ratio between radiometer output and incident radiation input (i.e., the output per unit input) is called the radiometer responsivity. There are three major kinds of radiometric responsivity since the scale used for calibration and measurement is established in terms of the radiometric quantity of interest at the reference location. If, as is frequently the case, the output, $\mathrm{V}$, is measured in volts, these three responsivities are (Section 5.2, Reference 9):

$$
\begin{aligned}
& \mathrm{R}_{\mathrm{P}} \equiv \mathrm{V} / \mathrm{P} \text {, the power responsivity, } \mathrm{v} \cdot \mathrm{w}^{-1} \\
& \mathrm{R}_{\mathrm{H}} \equiv \mathrm{V} / \mathrm{H} \text {, the irradiance responsivity, } \mathrm{v} \cdot \mathrm{w}^{-1} \cdot \mathrm{cm}^{2} \\
& \mathrm{R}_{\mathrm{N}} \equiv \mathrm{V} / \mathrm{N} \text {, the radiance responsivity, } \mathrm{v} \cdot \mathrm{w}^{-1} \cdot \mathrm{cm}^{2} \cdot \text { sterad }
\end{aligned}
$$

When the term "responsivity" (without the modifier) or "the symbol" (without the subscript) is used in this report, it refers equally well to any of these three quantities. In terms of the area of radiometer aperture, A, and field solid angle, $\boldsymbol{\Omega}$, these quantities are related in the ideal case as follows:

$$
\mathrm{R}_{\mathrm{P}}=\mathrm{AR} \mathrm{R}_{\mathrm{H}}=\mathrm{A} \boldsymbol{\Omega} \mathrm{R}_{\mathrm{N}}
$$




\subsection{RESPONSIVITY CALIBRATION}

In the ideal case being considered here the background and atmospheric transmission problems (which definitely must not be ignored in any actual calibration) are being ignored on the assumption that we can somehow obtain the equivalent of a background of zero radiance and a perfectly transparent atmosphere at all wavelengths of interest. We will concern ourselves, for the moment, only with the possible calibration configuration in terms of size and placement of the calibration source in relation to the radiometer. These are also treated in References 8 and 9 in a somewhat different manner.

2.3.1. DISTANT SMALL-SOURCE CALIBRATION. A small calibration source of radiant intensity $J$ is placed at a large distance S (see Figure 2) so that the image of the distant source falls entirely within the field stop of the instrument. In terms of rays, every source ray which passes through the aperture or entrance pupil of the instrument also passes through the field stop. The irradiance responsivity can then be computed directly without knowing either of the instrument optical parameters, A and $\Omega$, from the following relations:

$$
\mathrm{R}_{\mathrm{H}} \equiv \mathrm{V} / \mathrm{H}=\mathrm{VS}^{2} / \mathrm{J}
$$

If $\mathrm{A}$ and $\Omega$ are given, the power responsivity, $\mathrm{R}_{\mathrm{P}}$, and the radiance responsivity, $\mathrm{R}_{\mathrm{N}}$, are both readily computed from this result and the relations in Equation 2. It should be stressed that the foregoing applies only to an ideal situation, where $R$ does not vary across a sharply defined aperture and field and where background and atmospheric-transmission problems are assumed to have been eliminated. In any real situation, these must be considered, as will be discussed in later sections. In particular, see Section 3.6.1 of this report concerning background radiation.

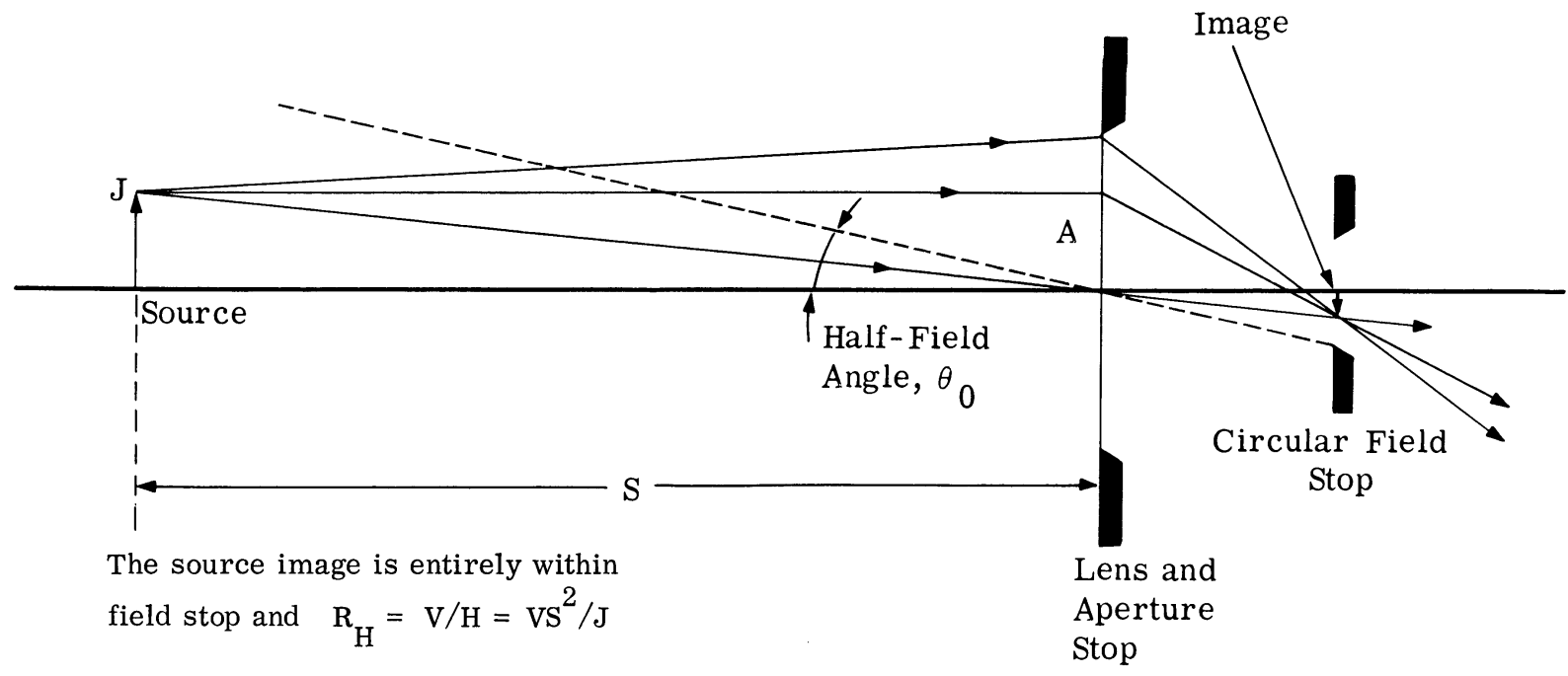

FIGURE 2. DISTANT SMALL-SOURCE CALIBRATION 
2.3.2. DISTANT EXTENDED-SOURCE CALIBRATION. A large calibration source of radiance $\mathrm{N}$ is placed at a distance sufficiently close to the instrument so that its image completely fills the field stop (see Figure 3). In terms of rays, a ray from any point in the field stop passing through any point in the aperture or entrance pupil arrives at the calibration source. In this case it is the radiance responsivity which can be computed directly, without knowing A or $\Omega$, from the defining relation (1). This is true because, neglecting transmission losses in our ideal case, the radiance, $\mathrm{N}$, at the aperture of the instrument or at the field stop, has the same value, $\mathrm{N}$, as it does at the source. This invariance of $\mathrm{N}$ along any ray is extremely useful in radiometric computations [ 8 and 9]. The power responsivity, $R_{P}$, and the irradiance responsivity, $\mathrm{R}_{\mathrm{H}}$, can then be computed from $\mathrm{R}_{\mathrm{N}}$ by Equation 2 .

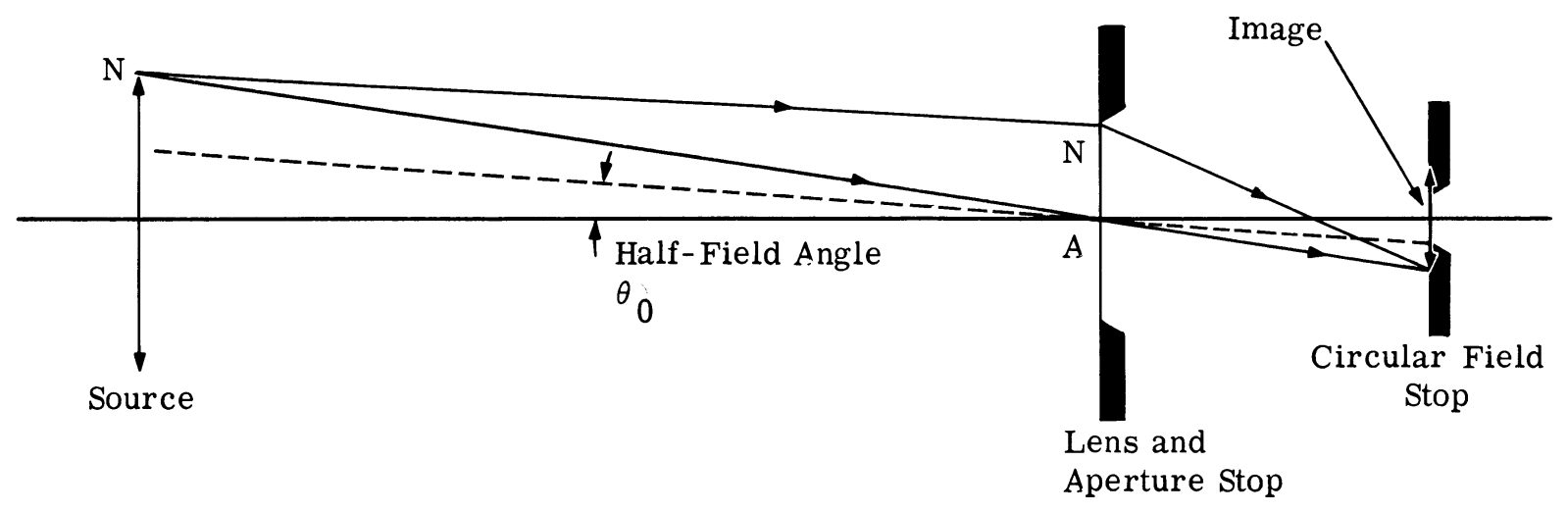

The source image completely fills the circular field stop and $\mathrm{R}_{\mathrm{N}} \equiv \mathrm{V} / \mathrm{N}$

\section{FIGURE 3. DISTANT EXTENDED-SOURCE CALIBRATION}

This method has particular value for measurements of extended targets. Thus, the spatial variation of radiance over targets such as ships, tanks, cars, buildings, airfields, and terrain can be determined best with instruments calibrated in this manner. An especially desirable practice is the location of extended calibration sources alongside the targets to be measured. In this case the atmospheric transmission is the same for target and calibration source. If the spectral distributions are sufficiently similar, a knowledge of the atmospheric transmission is no longer necessary for the calibration. On the other hand, a match of source spectral distributions or of paths to the radiometer may not be possible. The sun affords one example of such a source. Optically thin hot gases are also difficult to compare with conventional extended calibration sources. Rapidly moving sources, such as aircraft and ballistic missiles in flight, require a different calibration technique because of rapidly changing observational paths and spectral distributions difficult to match by calibration sources. 
2.3.3. NEAR EXTENDED-SOURCE CALIBRATION. A large extended source is placed directly in front of the radiometer aperture where it completely fills the aperture and field with radiation of uniform radiance, $\mathrm{N}$ (see Figure 4). The radiance responsivity, $\mathrm{R}_{\mathrm{N}}$, is then computed directly from Equation 1 , and both the power responsivity, $R_{P}$, and the irradiance responsivity, $R_{H}$, can be computed from it and the values of A and $\Omega$ by the relations (2). As noted in Figure 4, the source is not imaged at the field stop in this method. The radiometer should be focused exactly as it would be during the measurements of unknown source radiation.

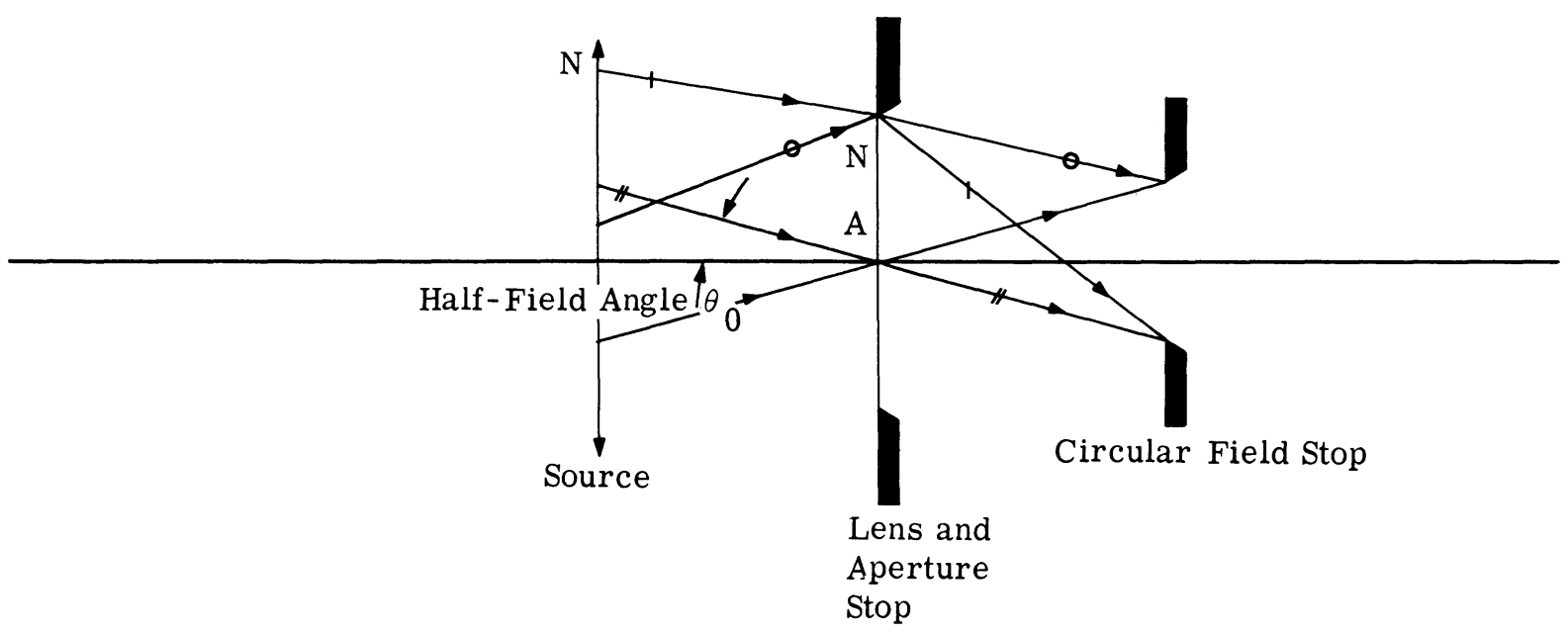

The source completely fills both the aperture and the field, but the image of the source is not at the field stop. Note that the rays which meet at the field stop are not parallel as they leave the source, $R_{N} \equiv V / N$.

\section{FIGURE 4. NEAR EXTENDED-SOURCE CALIBRATION}

\subsubsection{NEAR SMALL-SOURCE (JONES METHOD) CALIBRATION (see Section 5.3.4, Reference 9). A} source with an area small compared with the area of the radiometer aperture is placed close to the aperture and in a position where it will uniformly irradiate the field stop of the instrument (see Figure 5). Again, since the radiometer is focused for a target measurement, no image of the calibration source is formed at the field stop. The source must be close enough so that the radiometer aperture subtends an angle greater than the field angle of the instrument with respect to every point of the source. As can be seen in the figure, this means that the source must lie entirely within the region enclosed by the lines $\mathrm{XZ}$ and $\mathrm{YZ}$ which meet at $\mathrm{Z}$ to form an angle equal to the field angle of the radiometer. Under these conditions, each point of the source irradiates the field stop. However, only a small portion of the entrance aperture is used. In the nonideal case variations in transmittance across the entrance aperture must be evaluated.

Computation of the radiance responsivity is slightly more involved than in the previous cases. Consider an arbitrary point, $\boldsymbol{\rho}$, on the radiating surface of the source. It is apparent in Figure 5 that the rays emitted from $\boldsymbol{p}$ within a solid angle $\Omega$, equivalent to the field angle of the radiometer, will uniformly irradiate 
the entire circular field stop. If the radiance of the source is $\mathrm{N}_{\mathrm{S}}$ and its projected area is $\mathrm{A}_{\mathrm{S}}$, the radiant power from the source through the field stop (there are no losses in this ideal case) is given by

$$
\mathrm{P}=\mathrm{N}_{\mathrm{S}} \mathrm{A}_{\mathrm{S}} \Omega
$$

When the instrument is used for measurement of an extended source of radiance filling both the aperture and field (as in the near-extended source method of calibration), the total radiant power through the field stop is given by

$$
\mathrm{P}=\mathrm{NA} \Omega
$$

Thus the near small-source radiance, $\mathrm{N}_{\mathrm{S}}$, which will produce the same output, $\mathrm{V}$ (for which $\mathrm{P}$ is the same), as an extended source of radiance, $\mathrm{N}$, which fills the aperture and field, is given by

$$
\mathrm{N}_{\mathrm{S}}=\mathrm{AN} / \mathrm{A}_{\mathrm{S}} \text { or } \mathrm{N}=\mathrm{A}_{\mathrm{S}} \mathrm{N}_{\mathrm{S}} / \mathrm{A}
$$

The radiance responsivity is then given by

$$
\mathrm{R}_{\mathrm{N}} \equiv \mathrm{V} / \mathrm{N}=\mathrm{VA} / \mathrm{N}_{\mathrm{S}} \mathrm{A}_{\mathrm{S}}
$$

The power responsivity, $R_{P}$, and the irradiance responsivity, $R_{H}$, are again computed using $R_{N}$ and the values of A and $\Omega$ in relations (2). See Section 4.6.1, below, concerning background problems in connection with this method.

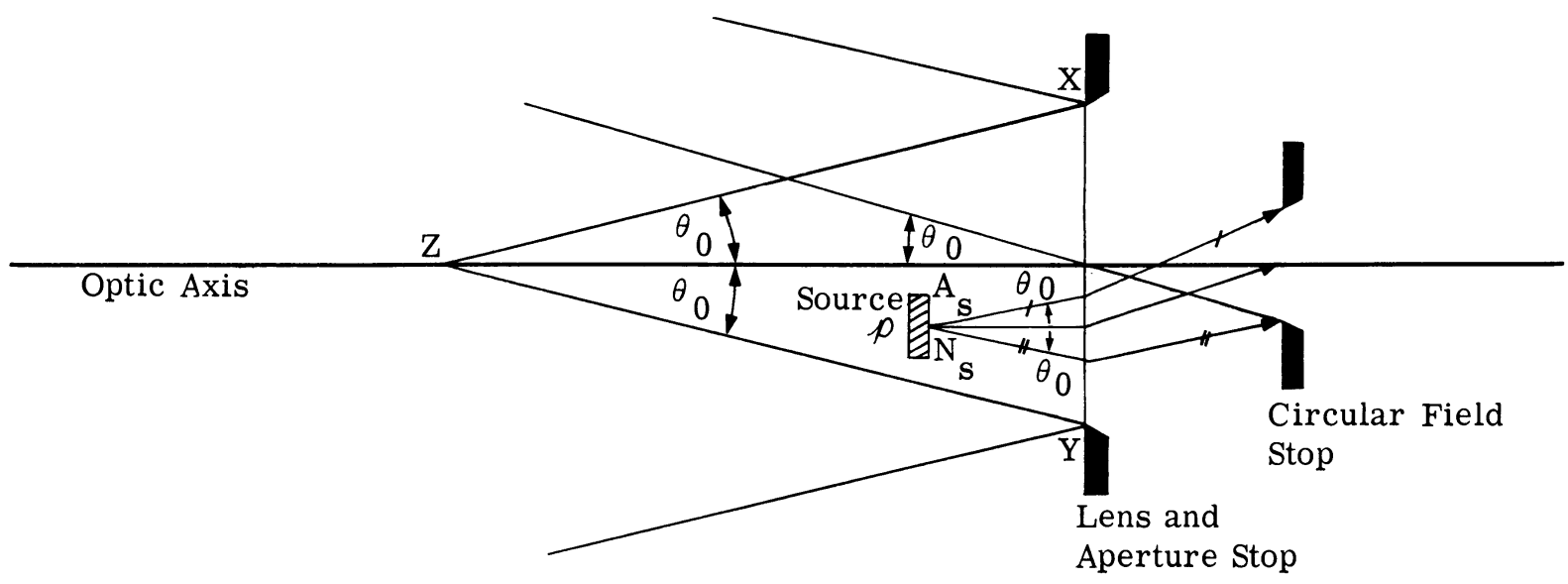

The source is completely within region bounded by $\mathrm{XZ}$ and $\mathrm{YZ}$, each of which make an angle $\theta_{0}$ with the optical axis where $\theta_{0}$ is the half-field angle. $\boldsymbol{\rho}_{\text {is }}$ any arbitrary point of the source. Rays from any such point, $\boldsymbol{P}$, within a cone of half-angle $\theta_{0}$ will uniformly irradiate the circular field stop as shown.

$$
\mathrm{R}_{\mathrm{N}}=\mathrm{V} / \mathrm{N}=\mathrm{VA} / \mathrm{A}_{\mathrm{S}} \mathrm{N}_{\mathrm{S}}
$$

where $A$ is the aperture area

$A_{S}$ is the source area

and $\quad \mathrm{N}_{\mathrm{S}}$ is the source radiance

FIGURE 5. NEAR SMALL-SOURCE (JONES METHOD) CALIBRATION 


\subsubsection{SUMMARY OF SOURCE SIZE AND PLACEMENT CONFIGURATIONS. In summary, we can} group the foregoing methods in different ways. The field stop is completely filled with radiation in the three methods used to obtain radiance responsivity directly; namely, the two extended-source methods and the near small-source or Jones method. In the distant small-source method the field stop is not filled with radiation. In this method it is the irradiance responsivity which is directly obtained.

As an alternative classification scheme, the methods may be grouped according to the location of the source image. In both of the near-source methods, the source image lies beyond the field stop. In each case care must be taken to make sure that the field stop lies wholly within the uniformly irradiated instead of the vignetted region of the radiation.

Finally, the methods may be grouped according to the source size. In the extended-source methods, both aperture and field are completely filled with radiation from the source. In the small-source methods either the aperture or the solid angle of field is filled with radiation. In the distant small-source method the aperture is filled but not the field. The reverse is true in the near small-source method. Thus there is opportunity in both small-source cases for background radiation to enter within the field solid angle (in the second case it would be through the remaining unused portion of the aperture). This possibility, which can produce an erroneous output, will be discussed in greater detail in Section 3.6.1, below.

2.3.6. USE OF A COLLIMATOR. The distant-source methods can be employed without actually placing the sources at great distances, if one places them at the focus of a collimator. The radiometer to be calibrated is positioned in the collimated beam, which then appears to be coming from a source at infinity. This has the advantage of keeping all of the components involved in the calibration conveniently accessible for manipulation and control. In addition, it makes it possible, in real situations, to control background radiation more easily and eliminate the effects of atmospheric attenuation by placing the source, collimator, and radiometer in an enclosure which can be flushed with dry nitrogen. Additional considerations involved in using a collimator will be discussed in Section 3.4.2.

2.3.7. REFERENCE RADIATION. As pointed out in the WGIRB Report, Section 4.3.1, the location of the zero on the scale established for the output of a radiometer by calibration measurements is frequently not specified although it is definitely a part of any complete calibration. Any report of measured values of radiometric quantities should include a clear statement of the reference radiation (i.e., the amount of incident radiation which would produce a zero output signal when the aperture and field of the radiometric instrument are uniformly filled by the incident beam). This value should be added to that computed from the radiometer output (and its responsivity) in order to obtain a result which is truly proportional to the incident radiant power at the reference position. In many cases, the reference radiation is insignificant in comparison with the incident radiation so that the difference is negligible; but, as emphasized in the WGIRB 
Report, this should be demonstrated, not assumed.

The determination of the reference radiation can be a rather subtle and elusive process. For example, the output of a thermocouple or thermopile is proportional to the difference between the junction or set of junctions in thermal contact with the blackened receiving element and a reference junction or set of junctions. It is the temperature of the latter which establishes the reference radiation. This quantity is not easily determined and may be only moderately constant, frequently depending upon the temperature of a heat sink which responds sluggishly to changes in ambient temperature.

In still other radiometers, sometimes called contrast radiometers, the output is a measure of the difference between the radiometric quantity for the beam from the target (and portion of the background) and that for the beam from an adjacent region or regions of the background. Here it is the radiation from the adjacent background that is the reference for the measurement of the radiation from the target (and a portion of the background). This reference often varies considerably for different measurements. A target of radiance equal to that of the background (zero contrast) would produce a zero output.

As a matter of convenience, the reference radiation is often specified in terms of the reference temperature of a radiometer. This is defined as the temperature of a blackbody which will produce a zero output when it completely fills the entire aperture and field of the instrument (when the aperture is large, this condition can be closely approximated by using a collimator). The use of temperature as a radiometric unit is discussed in Section 3.6.3.

3

\section{CALIBRATION OF A BASIC RADIOMETER: NON-IDEAL CASE}

Actual calibration and measurement situations, even using the simplest form of basic radiometer, can only approximate the ideal conditions which we consider in Section 2. It is therefore necessary to examine the ways in which real situations differ from the ideal and to determine which of these differences have significant effects on measurement results and how to deal with them. For a particular measurement or calibration, the only way to be sure that all of the possibilities have been covered adequately is to make tests, varying the different parameters and observing the effects of each on the radiometer output under controlled conditions. The possibilities are so numerous and vary so widely with the differences between the individual instruments and the situations in which they are employed, that it is not possible to cover them all in one general treatment. The discussion will therefore be limited to the more important of the effects most frequently encountered. 
We will concern ourselves especially with the effects of the three parameters representing spectral, spatial and temporal variations. The radiometric quantities to which a radiometer responds are, in general, functions of these parameters. Thus, the radiation at the radiometer aperture may be characterized by

or

$$
\begin{aligned}
& \text { the spectral radiant power, } \mathrm{P}_{\lambda}(\lambda ; \theta, \phi ; \mathrm{t}) \text { or } \mathrm{P}_{\lambda}(\lambda ; \xi, n ; \mathrm{t}) \\
& \text { the spectral irradiance, } \quad \mathrm{H}_{\lambda}(\lambda ; \theta, \phi ; \mathrm{t}) \text { or } \mathrm{H}_{\lambda}(\lambda ; \xi, n ; \mathrm{t})
\end{aligned}
$$

$$
\text { the spectral radiance, } \quad \mathrm{N}_{\lambda}(\lambda ; \theta, \phi ; \mathrm{t}) \text { or } \mathrm{N}_{\lambda}(\lambda ; \xi, n ; \mathrm{t})
$$

Here $\lambda$ is the symbol for wavelength. The spatial dependency is stated either in terms of rays, in reference to the optical axis and aperture stop of the radiometer by $\theta$ and $\phi$, or in terms of $\xi$ and $\eta$, cartesian coordinates in the projected image of the instrument field stop. The letters $\theta$ and $\phi$ symbolize the usual angular coordinates for a ray passing through the center of the aperture stop, as shown in Figure 6 . The coordinates, $\xi$ and $\eta$, have their origin at the intersection of the optic axis and the field stop image (see Figure 6). The temporal dependence is indicated by $t$, the symbol for time. In this report we frequently use the variables $\theta$ and $\phi$ rather than $\xi$ and $\eta$ to illustrate the value of specifying the spatial dependency of radiometer response in terms of directions rather than by coordinates in object space. Since an object and an image of finite dimensions are always present in radiometry, these forms of representation are completely equivalent. Radiometers with fields of view which are symmetric about the optical axis are most simply described by $\theta$ and $\phi$, while those with rectangular field stops are best treated with $\xi$ and $\eta$.

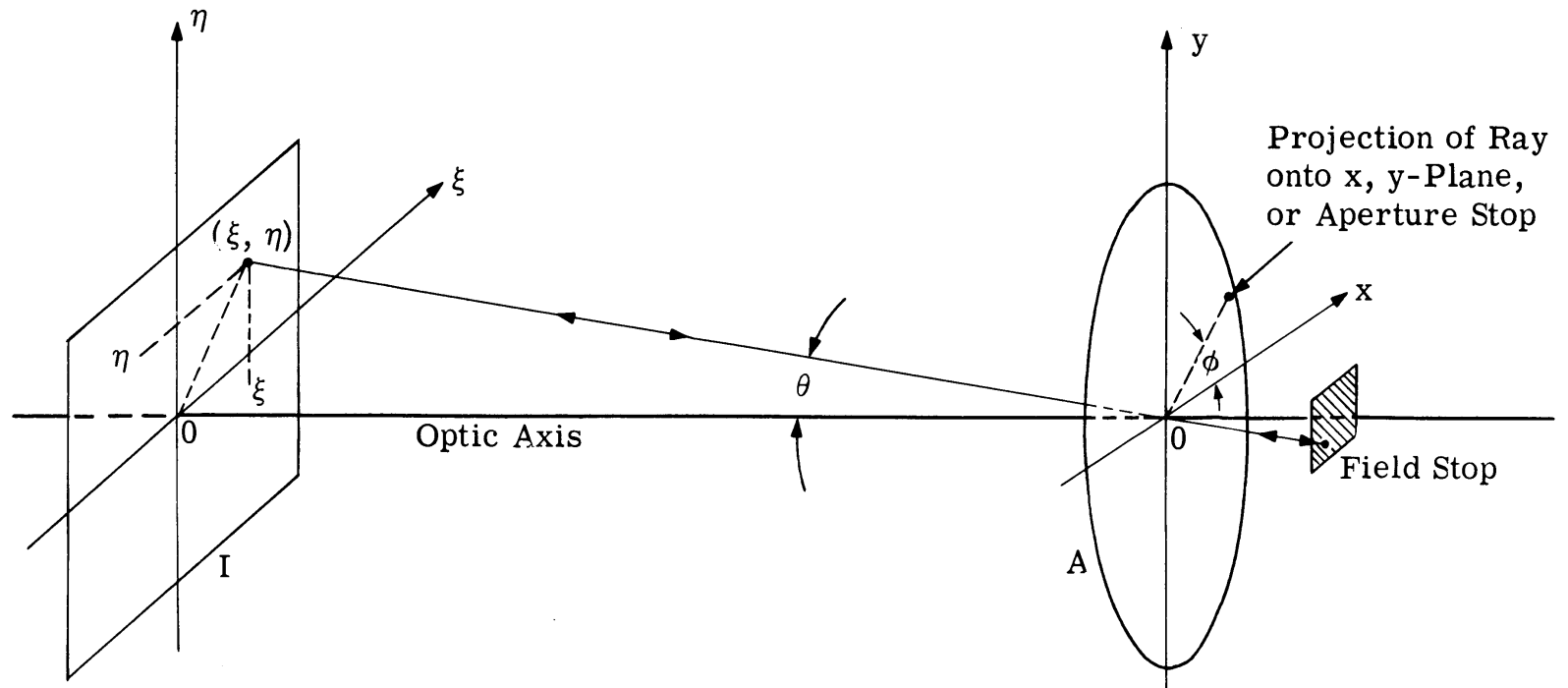

$I=$ The projected image of the field stop, shown without any distortions which might come from aberrations of the optics

$\xi, \eta=$ Cartesian coordinates in the field stop image plane

$\mathrm{O}=$ Origins of coordinate systems

$\theta, \phi=$ Angular coordinates of a ray passing through the center of the radiometer aperture

$\mathrm{A}=$ Radiometer aperture in the plane $\mathrm{x}, \mathrm{y}$

FIGURE 6. METHOD OF DESIGNATING DIRECTION OF RAY 
We will confine our attention in this section to measurements made with a basic radiometer and will consider more complicated and sophisticated instruments in later sections. Calibration will be considered for the measurement of the field quantities at the entrance aperture of the instrument. Problems such as atmospheric attenuation and background radiation, which can arise in connection with attempts to refer the calibration measurement results back to values existing at the source, will be deferred for later discussion.

\subsection{THE INSTRUMENT}

The three essential components present in every radiometer or radiometric instrument were listed in Section 2.1. In addition to those separate functions there are equally basic functions which, in the actual or non-ideal situation, usually involve interactions between the components. The wavelength interval in which the radiometric quantity is measured and the spectral responsivity of the radiometer within that interval (see Section 3.3) are ordinarily determined by the spectral characteristics of the optical components (including that of any filters and mirrors) combined with the spectral responsivity of the detector. The optical system alone (including, as specified, the sensitive surface of the detector) usually determines the directional or spatial dependence of responsivity, including the spatial resolution. The response to temporal fluctuations in incident radiation and the bandwidth or resolution in fluctuation frequency* measurements are determined by the detector element in combination with the amplifier and output indicator. However, if the responses to spectral or spatial variations of incident radiation are transformed into temporal fluctuations by some form of scanning (e.g., swinging the entire instrument for a continuous spatial scan, or changing filters with a rotating filter wheel to admit, successively, different narrow spectral bands) then the temporal frequency response may become a factor in determining also the spectral, or spatial, response and resolution.

It should be apparent from the foregoing that even the simplest form of radiometer cannot be calibrated accurately, nor can the results of measurements made with it be interpreted and evaluated without very complete and detailed information about the properties of all of its components and their functional relationships.

\subsection{INSTRUMENT RESPONSIVITY}

From the foregoing comments about the characteristics of real radiometers, it can be seen that the responsivities of radiometers are also functions of the parameters discussed, so that

*We recommend the use of $\mathrm{f}$ to refer to temporal frequency as distinguished from $\nu$, the frequency of the radiation itself, which is given in terms of wavelength and the velocity of light as $\nu=c / \lambda$. Similarly, we will use the words spectrum and spectral to refer only to distributions of wavelength, $\lambda$, and not to distributions of temporal or spatial frequencies, or of other quantities. 


$$
\mathrm{R}=\mathrm{R}(\lambda ; \theta, \phi ; \mathrm{t})
$$

This, together with Equation 8, means that, mathematically, the defining relations (Equation 1) in Section 2.2 can be evaluated only when a particular value is assigned to each of the parameters (i.e., for a single wavelength, direction, and time). But in reality there is no such thing as a precisely monochromatic and perfectly collimated beam of radiation, even from a laser. All real radiation fields consist of distributions of radiation over a range of wavelengths, directions, and usually also fluctuation frequencies. Particularly in the case of wavelength distributions, these are significant and may not be ignored in making radiometric measurements. Our problem then is to deal with responsivity in terms of the integrated effects of these distributions. Before looking at the net effect when all of the parameters may be changing simultaneously, which also includes possible interactions between the parameters, we will first discuss only one parameter at a time.

\subsection{SPECTRAL RESPONSIVITY}

It is relatively easy to minimize spatial responsivity problems by achieving nearly constant directional (spatial) response over a well-defined field solid angle. Similarly, temporal frequency response effects can be minimized by working with reasonably steady sources and using rapidly responding detectors with amplifiers and recorders of wide frequency response. However, it is almost impossible to eliminate entirely the spectral responsivity problems from the results of radiometric measurements.

In the case of a radiometer with an output, $\mathrm{V}$, which varies with the wavelength of the incident radiation, one must use the spectral power responsivity defined as

$$
\mathrm{R}_{\mathrm{P}_{\lambda}}(\lambda)=\operatorname{Lim}_{\Delta \lambda \rightarrow 0}\left[\frac{(\Delta \mathrm{V} / \Delta \lambda)}{(\Delta \mathrm{P} / \Delta \lambda)}\right]=\frac{\mathrm{V}_{\lambda}}{\mathrm{P}_{\lambda}}
$$

where

$$
\mathrm{V}_{\lambda}=\frac{\partial \mathrm{V}}{\partial \lambda} \text { in } \mathrm{v} / \mu
$$

and

$$
\mathrm{P}_{\lambda}=\frac{\partial \mathrm{P}}{\partial \lambda} \quad \text { in } \mathrm{w} / \mu
$$

The notation used is in keeping with the WGIRB recommendations concerning other wavelength-dependent quantities which are ratios, such as $\alpha(\lambda)$ or $\rho(\lambda)$. If no reasonable likelihood for confusion exists, the notation of $R_{P}(\lambda)$ may be used for the spectral power responsivity. The output voltage is given by

$$
\mathrm{V}=\int_{\lambda}^{\lambda} \mathrm{V}_{\lambda} \mathrm{d} \lambda=\int_{\lambda}^{\lambda} \mathrm{R}_{1} \mathrm{P}_{\lambda}(\lambda) \mathrm{P}_{\lambda}(\lambda) \mathrm{d} \lambda
$$


where $\lambda_{1} \leq \lambda \leq \lambda_{2}$ is chosen to include all wavelengths in which the integrands are not zero. These relations are illustrated in Figure 7 . We can also define a useful quantity, the relative spectral power responsivity, $\mathrm{L}_{\mathrm{P}}(\lambda)$, as the spectral power responsivity divided by a normalizing constant. For example,

$$
L_{P}(\lambda)=\frac{{ }_{P_{\lambda}}(\lambda)}{R_{P_{\lambda}}\left({ }_{\text {max }}\right)}
$$

where $\lambda_{\max }$ is the wavelength at which $\mathrm{R}_{\mathrm{P}_{\lambda}}(\lambda)$ is a maximum.

It should be noted that $R_{P_{\lambda}}(\lambda) \neq \frac{\partial R_{P}}{\partial \lambda}$. Instead we have

$$
\mathrm{R}_{\mathrm{P}}=\frac{\mathrm{V}}{\mathrm{P}}=\frac{\int_{\lambda}^{\lambda}{ }^{\lambda} \mathrm{V}_{\lambda}(\lambda) \mathrm{d} \lambda}{\int_{\lambda}^{\lambda} \mathrm{P}_{\lambda}(\lambda) \mathrm{d} \lambda}=\frac{\int_{\lambda}^{\lambda} 2 \mathrm{R}_{\mathrm{P}_{\lambda}}(\lambda) \mathrm{P}_{\lambda}(\lambda) \mathrm{d} \lambda}{\int_{\lambda{ }_{1}}^{\lambda} \mathrm{P}_{\lambda} \mathrm{d} \lambda}
$$

Thus $R_{P}$ is related to $R_{P_{\lambda}}(\lambda)$ by a calculation which involves the spectral radiant power as a weighting function. As a result, the value of $R_{P}$ depends upon the wavelength distribution of the radiation used in the calibration. The spectral irradiance responsivity and the spectral radiance responsivity can be treated similarly.

3.3.1. NORMALIZATION. The next step in applying the results of the preceding section involves the idea of normalization. In the above instance, as will be seen, we have normalized to the peak of the spectral responsivity. Assume that the value of $R_{P_{\lambda}}\left(\lambda_{m}\right)$ has been determined from Equation 11 by a calibration measurement with a certain instrument for which $\mathrm{L}_{\mathrm{P}}(\lambda)$ is known and with a calibration configuration for which $\mathrm{P}_{\lambda}(\lambda)$ is also known. (The problem of measuring the spectral distributions $L_{P}(\lambda)$ and $P_{\lambda}(\lambda)$ will be discussed in later sections.) The instrument is then placed in an unknown beam of radiation, and the output, V, is obtained. From this value of $V$, and the value of $R_{P}\left(\lambda_{m}\right)$ in the relations of Equation 11 , it is possible to obtain only the valuable of the integral

$$
V / R_{P}\left(\lambda_{m}\right)=\int_{\lambda}^{\lambda} P_{\lambda}(\lambda) L_{P} d \lambda
$$




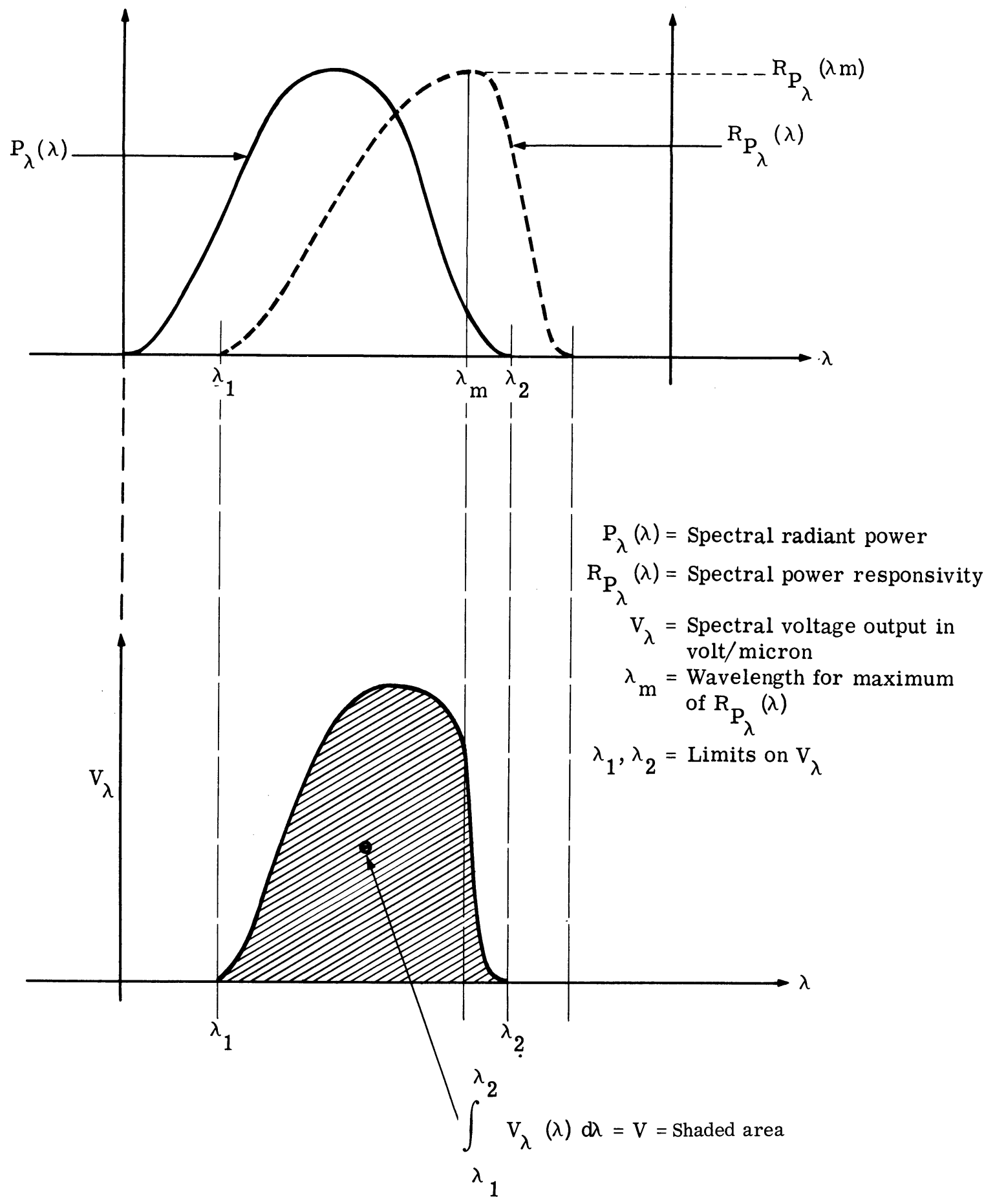

FIGURE 7. SPECTRAL POWER RESPONSIVITY AND SPECTRAL OUTPUT 
instead of the desired measure of the power in the unknown beam,

$$
\mathrm{P}_{\Delta \lambda}=\int_{\lambda}^{\lambda} \mathrm{P}_{\lambda}(\lambda) \mathrm{d} \lambda
$$

Equation 13 defines the radiant power normalized to the peak of the radiometer spectral responsivity. Note that this quantity does not contain information about the radiometric quantity alone, but is the result of the interaction between the radiometric quantity and the instrument spectral response. It tells us nothing more about an unknown spectral distribution of incident radiation than the response which can be expected from another instrument with the same relative spectral responsivity at all wavelengths. To illustrate, the output of an instrument will be the same for $100 \mathrm{w}$ incident at a wavelength where its response is $10 \%$ of the peak response, or for $1000 \mathrm{w}$ at a wavelength where its response is $1 \%$ of the peak. Normalized to the peak, both responses can be interpreted only as the equivalent of $10 \mathrm{w}$ incident at the wavelength of peak response; but the same would be true for another instrument only if it had the same spectral responsivity at all of these wavelengths, not just the same value of peak spectral responsivity. Even with the most careful and complete calibration of a radiometer with nonuniform spectral responsivity, one can never exactly achieve the goal of making the measurement result, by itself, completely independent of the measuring instrument. The importance of reporting the instrument spectral responsivity whenever data are reported should be obvious.

In a great many cases, independence can be achieved on the basis of additional information about the spectral distribution of the incident radiation - i.e., about the quantity

$$
r(\lambda)=P_{\lambda}(\lambda) / P_{S}
$$

Here $P_{S}$ is a scale factor which may be $P_{\lambda}\left(\lambda_{m}\right)$ or $P_{\lambda \text {,av }}$. However, without additional information or assumptions, the statements in the preceding paragraph represent the limitations on the information to be derived from $\mathrm{V}$ alone, even with the best possible calibration.

For example, it may be known (or assumed) that the relative spectral distribution of power in the interval $\lambda_{1} \leq \lambda \leq \lambda_{2}$ for incident radiation from an unknown source is the same as that in the beam from the source used for calibration (for which the absolute value, as well as the distribution, is known). In that case, the power in that wavelength interval from the unknown source can be expressed as

$$
\mathrm{P}_{\Delta \lambda}=\int_{\lambda}^{\lambda} \mathrm{P}_{\lambda}(\lambda) \mathrm{d} \lambda=\mathrm{P}_{\mathrm{s}} \int_{\lambda_{1}}^{\lambda_{2}} \mathrm{r}(\lambda) \mathrm{d} \lambda=\mathrm{P}_{\mathrm{s}} \Delta \lambda
$$


where $r(\lambda)$ is the relative spectral distribution of the radiation

$\mathrm{P}_{\mathrm{S}}$ is the scale factor

As discussed in Reference 8 and in Sections 4.3.3.1 and 4.3.3.2 of Reference 9, the value of $P_{S}$ and the maximum value of $r(\lambda)$ will depend on the method of normalization employed. The value of the integral

$$
\int_{\lambda}^{\lambda} r(\lambda) d \lambda=\Delta \lambda
$$

is the spectral interval or bandwidth over which a constant value, $\mathrm{P}_{\mathrm{S}}$, would present the same total power as the integral of the spectral radiant power from $\lambda_{1}$ to $\lambda_{2}$, i.e.,

$$
\mathrm{P}_{\Delta \lambda}=\int_{\lambda}^{\lambda} \mathrm{P}_{\lambda}(\lambda) \mathrm{d} \lambda=\mathrm{P}_{\mathrm{S}} \int_{\lambda}^{\lambda+\Delta \lambda} \mathrm{d} \lambda=\mathrm{P}_{\mathrm{S}} \Delta \lambda
$$

If, in fact, $\mathrm{P}_{\mathrm{S}}$ is the maximum value of $\mathrm{P}_{\lambda}(\lambda)$ (as in normalization to the peak), then the value of bandwidth given by Equation 15 must be labelled the peak-normalized bandwidth. Obviously, if $P_{S}$ is the average value of $\mathrm{P}_{\lambda}(\lambda)$ in the interval $\lambda_{1} \leq \lambda \leq \lambda_{2}$, then the bandwidth given by Equation 15 will be that of the entire interval, $\Delta \lambda=\lambda_{2}-\lambda_{1}$. In the case of normalization to the average the power in the incident beam is given by:

$$
\mathrm{P}_{\Delta \lambda}=\int_{\lambda{ }_{1}}^{\lambda} \mathrm{P}_{\lambda}(\lambda) \mathrm{d} \lambda=\mathrm{P}_{\lambda, \mathrm{av}} \int_{\lambda}^{\lambda} \mathrm{r}(\lambda) \mathrm{d} \lambda=\mathrm{P}_{\lambda, \mathrm{av}}\left(\lambda_{2}-\lambda_{1}\right)=\mathrm{P}_{\lambda, \mathrm{av}} \Delta \lambda
$$

We write the relationships in terms of the responsivity obtained from a previous calibration since we know (or have assumed) that the unknown source radiation has the same spectral distribution at the reference position as the radiation from the calibration source. From Equations 10, 11, and 17 we can write (for the unknown source):

$\left.\mathrm{V}=\int_{\lambda}^{\lambda} \mathrm{P}_{\lambda}(\lambda) \mathrm{R}_{\mathrm{P}_{\lambda}}(\lambda) \mathrm{d} \lambda=\mathrm{P}_{\lambda, \mathrm{av}} \mathrm{R}_{\mathrm{P}_{\lambda}}\left(\lambda \mathrm{m}^{\prime}\right) \int_{\lambda}^{\lambda} \mathrm{r}(\lambda) \mathrm{L}_{\mathrm{P}}(\lambda) \mathrm{d} \lambda=\left(\mathrm{P}_{\Delta \lambda} / \Delta \lambda\right) \mathrm{R}_{\mathrm{P}_{\lambda}}{ }_{\lambda}{ }_{\mathrm{m}}\right) \int_{\lambda}^{\lambda} \mathrm{r}(\lambda) \mathrm{L}_{\mathrm{P}}(\lambda) \mathrm{d} \lambda$

From our calibration we have

$$
R_{P}=V / P=\left[R_{P_{\lambda}}\left(\lambda_{m}\right) / \Delta \lambda\right] \int_{\lambda_{1}}^{\lambda} r(\lambda) L_{P}(\lambda) d \lambda
$$


and so we can compute

$$
\mathrm{P}_{\Delta \lambda}=\mathrm{V} / \mathrm{R}_{\mathrm{P}}
$$

Thus it is possible to employ a spectrally selective instrument to measure exactly the power, $\mathrm{P}_{\Delta \lambda}=\mathrm{P}_{\mathrm{S}}$ $\int_{\lambda_{1}}^{\lambda} \mathrm{r}(\lambda) \mathrm{d} \lambda$, in an unknown beam of radiation only under the very special circumstance that the instrument is calibrated with a known beam of radiation which has the same spectral distribution, $r(\lambda)$. In that case, the value of $R_{P}$ determined from Equation 12, which is the first part of Equation 18, can be used together with the output, $\mathrm{V}$, produced by the unknown radiation, to compute the power, $\mathrm{P}_{\Delta \lambda}$, in the unknown beam by Equation 19.

Incidentally, this is a good illustration of the applicability of the general principle stated in the Introduction: to conduct calibration measurements under conditions which reproduce, as completely as possible, the circumstances under which measurements based on that calibration are made. This clearly applies to the spectral distribution of radiation in each situation. On the other hand, it should be recognized that, as a practical matter, it may be extremely difficult or impossible to reproduce the spectral distribution of targets of interest in the radiation from calibration sources for which the values are known. Blackbody sources are among those most frequently employed for calibration measurements because the values of the radiometric quantities for the emitted radiation are more easily determined than for most other sources. However, the radiation beams from many sources to be measured, particularly those at distances where the radiation is strongly attenuated by the atmosphere, have a spectral distribution at the radiometer which varies widely from the blackbody. As a result, the goal of making the spectral distribution of incident radiation the same for both calibration and measurement becomes one that is desirable but often very difficult to attain.

Perhaps it should also be mentioned that, when the spectral responsivity of a radiometer is nearly constant over the spectral range in which it responds, the radiant power of an incident beam of radiation can be measured with an uncertainty which depends upon the constancy of $\mathrm{R}_{\mathrm{P}_{\lambda}}(\lambda)$. Note, however, that it is absolutely impossible to estimate the degree of uncertainty in this situation without some information (actual or assumed) about the spectral distribution of radiation in the incident beam.

All of the foregoing discussion in this section has been in terms of power, P, and power responsivity, $\mathrm{R}_{\mathrm{P}}$. However, it applies equally well to other radiometric quantities if $\mathrm{H}$ or $\mathrm{N}$ is everywhere substituted for $P$, and $R_{H}$ or $R_{N}$ is substituted for $R_{P}$. Note that $r(\lambda)$, for the same incident radiation, is not affected by this shift from one radiometric quantity to another.

The method of normalization to the peak is the one most frequently employed; but there are situations where it is not appropriate to use it and where other schemes are used, as in Equation 17. These other 
methods will not be discussed further here. Two examples are presented in some detail in Section 4.3.3.2 of Reference 9. Because of these possible variations in methods of normalization, the use of normalized values in a report of measurements or calibrations without specification of the way in which they were normalized, can lead to ambiguity and misinterpretation. We therefore repeat and concur in the recommendation given in the cited section of the WGIRB Report: "It is important that the method of normalization be carefully described."

3.3.2. SPECTRAL RESPONSIVITY CALIBRATION. Although it should be clear from the foregoing that an accurate measure of the spectral responsivity of a radiometer is a very important factor in interpreting measurement results, this factor is probably one of the most neglected phases of calibration. One reason for this is that it is among the most difficult. The difficulties, which are well summarized in the WGIRB Report, Section 5.7, can be seen from the following considerations.

Two approaches can be taken to obtain the spectral responsivity of a radiometer with a relatively broad spectral passband (the calibration of spectroradiometers with narrow instantaneous spectral passbands will be discussed in a later section). One approach is to compute the overall spectral responsivity from data for the spectral characteristics of all of the components involved. The other is to make direct measurements of the relative responsivity of the complete radiometer as a function of wavelength.

There are numerous pitfalls in the first method. Most of the important ones are mentioned in the WGIRB Report in the section cited above. Manufacturers generally do not keep the variability of the spectral characteristics of detectors and filters within narrow ranges. Published curves, particularly those used for advertising purposes which apply to "typical" items, frequently differ substantially from the characteristics of a particular sample. Another pitfall lies in the possibility of interaction between components, such as multiple reflections between adjacent parallel surfaces. The transmittance of such a combination may not be just the product of the separately measured transmittances.

In keeping with the general principle of making calibration reproduce, as closely as possible, the conditions to be used in making measurements, a spectral-responsivity calibration of the entire instrument, as a unit, is to be preferred wherever it is feasible. This can be done by observing the response of the radiometer to the radiation from the exit slit of a good monochromator as a function of the wavelength setting. The beam from the monochromator is also measured as a function of the wavelength setting with a reference detector, with responsivity substantially independent of the wavelength (a so-called black detector). The relative spectral responsivity curve of the radiometer is obtained by taking the ratio of the resulting radiometer response to the output of the reference detector.

For reliable measurements of the entire passband, particularly of the degree of rejection for wavelengths outside of the region of substantial responsivity, it is important that the monochromator beam be 
one of high spectral purity. Thus, a double monochromator is preferable. Supplemental filters may also be used with it to insure against the presence of small amounts of radiation of undesirable wavelengths. At these wavelengths the instrument responsivity is so much greater than it is at the wavelength setting of the monochromator that even a very small amount would otherwise produce a significant error in the radiometer output.

The WGIRB Report points out that there may be difficulty in obtaining enough radiant power from the exit slit of the monochromator and suggests that a collimator may be used (see comments in Sections 2.3.6 and 3.4.2 of this paper). When this is done, it probably will not be feasible to place the reference detector in the same beam from the collimator because, small in size, it probably would not intercept a sufficient fraction of the available radiant power. Instead it will probably be necessary to use a two-position mirror to direct an image of the exit slit of the monochromator alternately onto the field stop of the collimator and onto the reference detector. In that case it is highly important to have the two positions of this mirror mechanically rigid and accurately reproducible. In addition, every effort should be made to insure that the spectral quality of the beam incident on the reference detector exactly matches that of the collimated beam on the radiometer, which will involve introducing the same number of reflections from the same types of mirror surfaces, considering polarization effects, and taking other precautions. If this cannot be done, the differences should be carefully analyzed and accounted for, keeping in mind that a mirror has a spectral emissivity equal to one minus its spectral total hemispherical reflectance which, at the longer wavelengths, can result in significant self-emission from mirror surfaces at room temperature. In any event, where the two beams take different paths which have been designed to be similar, it is wise to redetermine the reflectivity of the mirrors from time to time to see that differences have not arisen through uneven soiling or gradual deterioration.

Finally, a word of caution about the black detector: since this is the reference standard, its accuracy controls the accuracy of the spectral-responsivity calibration. If possible, the spectral absorptance (equal to one minus the total spectral reflectance) should be checked by a measurement of the total spectral reflectance. Note that it is not the specular, but the total or hemispheric reflectance that is required. As nearly as possible, the incident beam in the reflectance measurements should match the geometry of the beam from the monochromator in the spectral responsivity calibration.

In view of the improbability that the beam exiting from a monochromator will possess the required area and solid angle to perform absolute spectral responsivity calibrations, we strongly urge that only a relative spectral responsivity calibration be made in this manner. Absolute values at selected wavelengths can be obtained by the appropriate use of narrowband filters and a blackbody in a separate set of calibration measurements. 


\subsection{SPATIAL RESPONSIVITY}

At the entrance aperture of the radiometer, as indeed at any aperture, there are two ways in which radiation may be distributed in space. First, there is a spatial distribution of irradiance at different points (elements of area) across the area of the aperture. Second, there is a directional distribution on the irradiance at any one point due to radiation coming from different elements of solid angle. In most measurement configurations, where a radiometer is used to measure the radiation from a more-or-less distant source, the value of irradiance, $\mathrm{H}$ (or radiance, $\mathrm{N}$ ), is the same at all points across the aperture. In these cases, we are seldom concerned with problems involving a spatial distribution of the first kind. On the other hand, it is only when a source of uniform radiance fills the entire field of the instrument that the angular or directional distribution of radiation is uniform in all directions within the field solid angle. This directional distribution can be thought of in terms of the spatial distribution across the image plane when the instrument is focused so that the image plane and the field stop coincide.

Before leaving the first type of spatial distribution, we should point out that there are instances where it may be a significant factor. For example, when a collimator is used with a calibration source, as suggested in Section 2.3.6 above, it is essential to have a uniform distribution of radiation across the collimated beam incident on the entrance aperture of the radiometer. If the beam is uniform and fills the radiometer aperture, the output will be directly proportional to the irradiance, even though the radiometer's responsivity may vary across its aperture (e.g., because of variations in the reflectance caused by dirt or deterioration of the surface across a primary mirror). If the beam is not uniform or if it fills only a part of the radiometer aperture, then the radiometer's output response will vary with the relative positions of the two apertures (even with the optical axes of collimator and radiometer always parallel). It then becomes impossible to assign a meaningful value to the irradiance at the reference position or to determine $R_{H}$, the responsivity to uniform irradiance across the entire radiometer aperture.

In the near small-source or Jones method of calibration there is an assumption that the radiometer responds uniformly to radiation received through all portions of its aperture. Section 2.3.4 and Figure 5 , particularly, show that radiation from any point of the source to the field stop passes through only a portion of the radiometer aperture. Furthermore, this portion is different for each different point of the source. Consequently, the net or total power passing through the field stop to the detector will vary with the position of the source if, for example, a collecting mirror has non-uniform reflectance or if its focal length varies across the aperture ([9] Section 5.3.4).

3.4.1. DIRECTIONAL RESPONSIVITY - USE OF A FIELD LENS. Unfortunately, a great many radiometric measurements are made with instruments which do not respond uniformly to radiation coming from different directions within a sharply defined angular field. This is most often due to the fact that, in the simplest form of radiometer, the detector is located in the image plane of the collecting optics where it 
serves as the field stop. Any nonuniformity in responsivity across its sensitive surface, combined with the effects of any optical aberrations, produces a varying response to rays reaching the instrument aperture from different directions.

In many cases it is possible to achieve a more nearly ideal directional response by the use of a field lens. A field stop in the image plane of the primary optics is used to sharply define the angular field. A field lens placed close to this field stop images the entrance aperture on the surface of the detector. Since, as pointed out above, the entrance aperture is uniformly irradiated under most circumstances, even though the field is not, a nearly uniform irradiation of the detector is produced regardless of variations across the field stop. However, it is well to bear in mind that the uniformity of directional response has been obtained at the expense of possible nonuniformity of spatial response across the entrance aperture. This could be a significant problem, for example, in attempting to calibrate such an instrument by the near smallsource or Jones' method. It should also be noted that the success of this use of a field lens is greatly dependent on the quality of that lens. Substantial aberrations can do much to reduce its effectiveness in producing uniform directional response over the field. Finally, the spectral transmittances of available materials for such field lenses place limitations on the cases in which this is a feasible approach. We strongly concur with the recommendation in Section 5.5 of the WGIRB Report: "This configuration is recommended when wavelength considerations permit."

3.4.2. MAPPING THE FIELD. The field of view of a radiometer should always be measured and the results included in the calibration data, which should be a part of any report of measurement results based on that calibration. An exception can be made when the instrument is used only to measure the radiance of uniform extended sources and where, for all of the measurements including the calibration, there is clearly no question that the entire field is filled with radiation from the uniform source. In that case there is no need for detailed information about the exact size of the field nor about the distribution of directional response within the field. However, if the field extends over regions of substantial variation in a nonuniform extended source, and it is desired to obtain meaningful measurements of average radiance over these regions, a uniform directional response across a sharply defined field is essential and should be verified as part of the calibration.

The field can be mapped with any convenient small source of constant radiant intensity (for relative measurements the value of $J$ need not be known) at a distance where its image covers only a very small portion of the field stop. The size of this image determines the resolution with which the variation of directional responsivity can be determined. The size should be limited by the circle of least confusion of the collecting optics of the radiometer. The source can be moved horizontally and vertically in a plane perpendicular to the optic axis of the radiometer, or the radiometer can be scanned across the source (see Figure 8). Section 5.5 of the WGIRB Report discusses methods of presentation of the field of view which 
should be compared with this and the discussion in 3.4 .3 below.

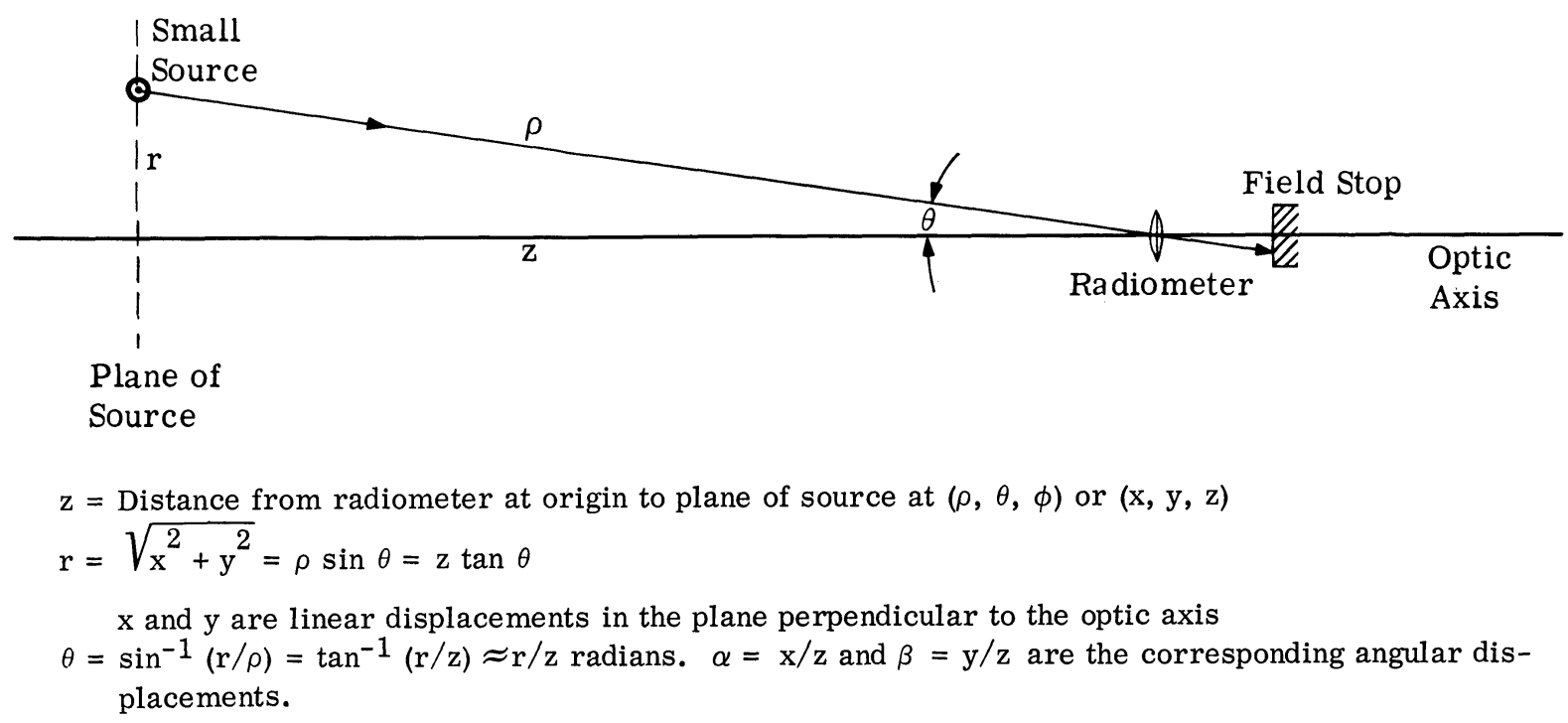

FIGURE 8. GEOMETRY FOR MAPPING THE FIELD WITH A DISTANT SMALL SOURCE

The distance of the small source must also be sufficient to insure that it is in sharp focus in the field stop if, as is frequently the case, the instrument has fixed focus optics. If a shorter distance is used with an instrument with adjustable focus, it is wise to check the measurements at more than one distance to make sure that they are not affected by the focusing. This should be done especially before applying the results to measurements of sources at ranges different from those at which the calibration measurements are made.

A collimator may also be used for mapping the field of a radiometer. The size of the source in the focal plane of the collimator (the collimator field stop) must be adjusted to produce the desired size of source image in the field stop of the radiometer. For ideal optics, with no aberrations, the source and its image will subtend the same angle with respect to the center of the aperture stop of their own optical system (the angle $\psi$ in Figure 9). The magnification of the system is the ratio between the source and its image in the radiometer, which is the same as the ratio between the focal lengths of the radiometer and collimator optics. The collimator should be of good enough optical quality so that it does not degrade the results of the measurement. The blur circle of the collimator, measured in terms of the angle which it subtends at the center of the collimator aperture stop, should be small in relation to the blur circle of the radiometer, also measured by the angle which it subtends at the radiometer aperture. Under these circumstances the minimum image size is determined by the aberrations of the radiometer optics.

The collimator is positioned so that, as the angle $\alpha$ between its optic axis and that of the radiometer is varied, the two axes always intersect at 0 , the center of the radiometer aperture stop. This arrangement will keep the aperture of the radiometer filled, as shown in Figure 9. The figure has been drawn showing 


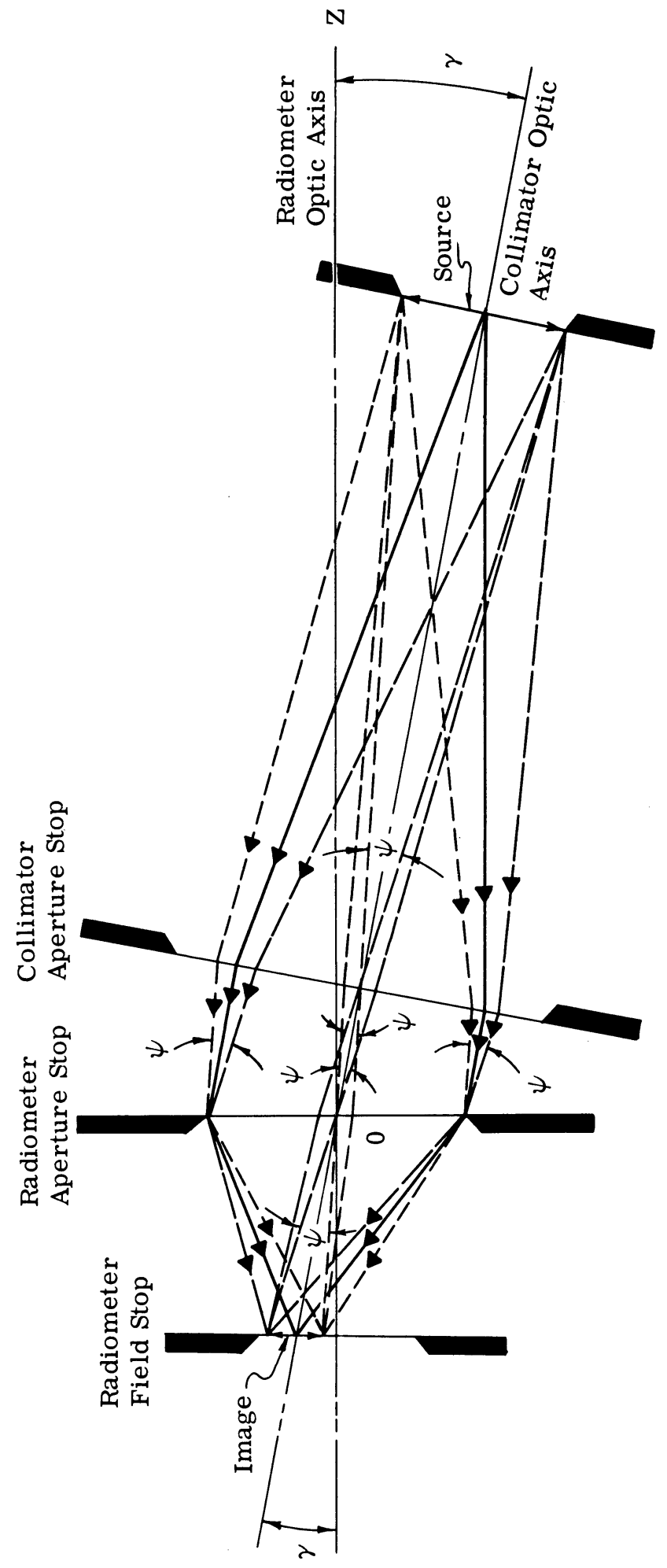

要 
the paths of the extreme rays through the radiometer aperture to illustrate the need (even with perfect alignment) for a collimator aperture somewhat larger than that of the radiometer.

3.4.3. COMPUTATION OF EFFECTIVE FIELD SOLID ANGLE. We will define the effective field solid angle as that value of $\Omega$ which will satisfy the relation of Equation 2, even for a non-ideal radiometer. In other words, it is the ratio between the irradiance responsivity and the radiance responsivity of the instrument. If there is nonuniform directional response, so that the ir adiance responsivity varies with direction, it is necessary to be more specific. Note that the value which satisfies Equation 2 should be designated as the effective field solid angle with respect to the direction corresponding to the maximum irradiance responsivity.

Before looking at this definition of effective field solid angle in more detail, it may be desirable to review the computation of the purely geometrical solid angle subtended at 0 , the center of the aperture stop, by a circular field stop (see Figure 10). The exact value of the subtended solid angle is given by

$$
\Omega=\int_{0}^{2 \pi} \int_{0}^{\theta} \sin \theta \mathrm{d} \theta \mathrm{d} \phi=2 \pi\left(1-\cos \theta_{0}\right)
$$

where the symbols are defined in the figure. However, a useful and frequently employed approximation is to treat distances in the plane of the field stop as if they were directly proportional to the angle they subtend at the origin. Thus, approximations are used, such as:

$$
\mathrm{r}=\rho \sin \theta \approx \rho \theta
$$

With this approximation, the solid angle becomes

$$
\Omega \approx \pi \mathrm{r}^{2} / \rho^{2}=\pi \theta_{0}^{2}
$$

The range over which this approximation may be used for a desired degree of precision is readily evaluated. If we expand the cosine into a power series, Equation 20 becomes

$$
\begin{aligned}
\Omega & =2 \pi\left(1-1+\frac{\theta_{\mathrm{o}}^{2}}{2 !}-\frac{{ }_{\mathrm{o}}}{4 !}+\frac{{ }_{\mathrm{o}}}{6 !}-\ldots\right) \\
& =\pi\left(\theta_{\mathrm{o}}{ }^{2}-\frac{\mathrm{o}}{12}+\frac{{ }_{\mathrm{o}}}{360}-\ldots\right)
\end{aligned}
$$

It is immediately apparent that the approximation of Equation 22 amounts to retaining only the first term of this series expansion. The error involved in dropping all of the terms beyond a certain point in a convergent alternating series is equal to or less than the magnitude of the first term to be dropped. Hence, for $1 \%$ accuracy of approximation, we may set 


$$
\begin{gathered}
\frac{\theta_{\mathrm{o}}^{4}}{12} \leq 0.01 \theta_{\mathrm{o}}{ }^{2} \\
\theta_{\mathrm{o}} \leq \sqrt{0.12}=0.347 \mathrm{rad}=19.8^{\mathrm{o}}
\end{gathered}
$$

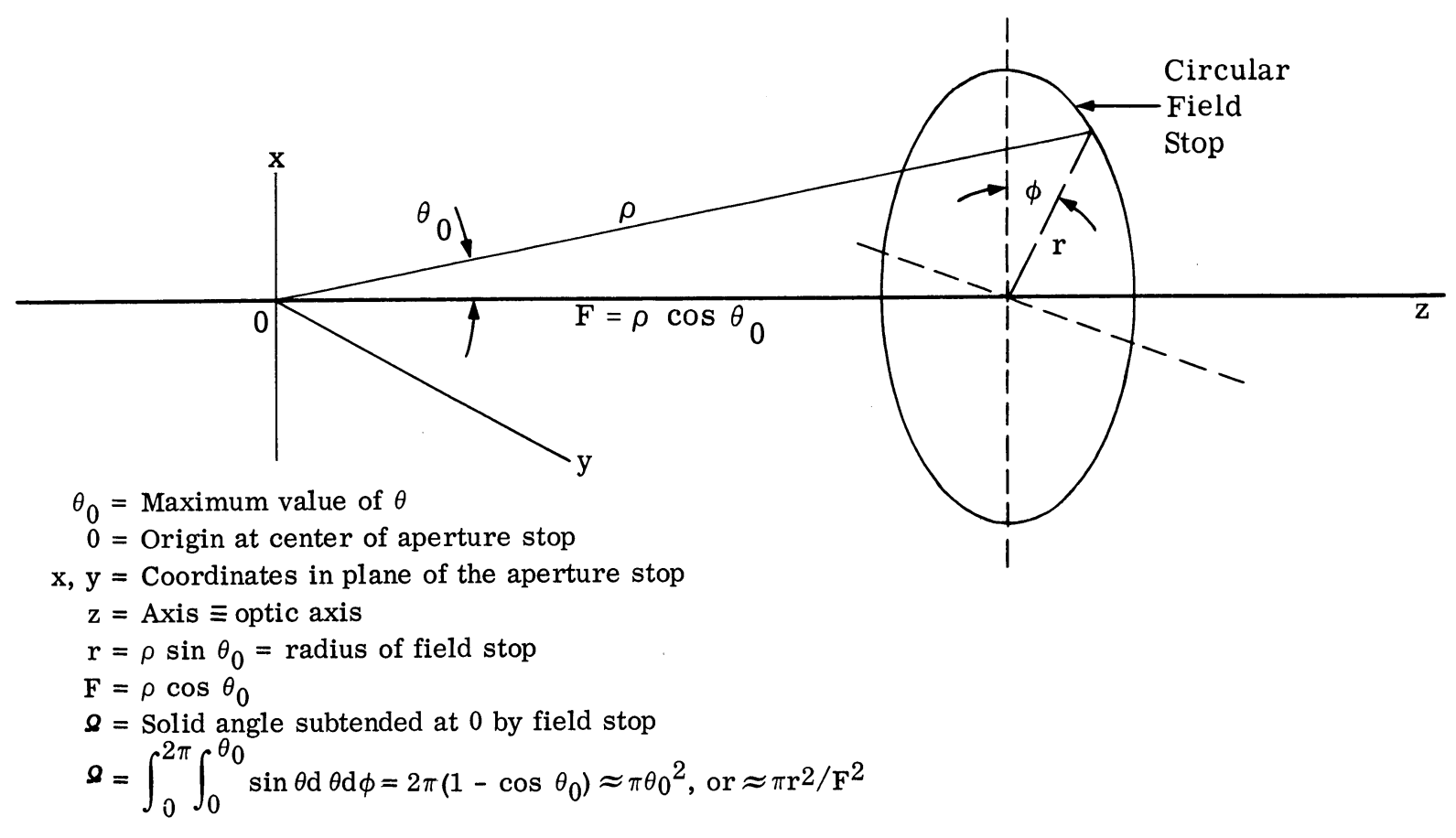

FIGURE 10. SOLID ANGLE OF CIRCULAR FIELD STOP

Another approximation, probably even more commonly used, is the computation of the solid angle from

$$
\Omega \approx \pi \mathrm{r}^{2} / \mathrm{F}^{2}=\pi \tan ^{2} \theta_{0}
$$

Again using series expansion, we can write

$$
\begin{aligned}
& \tan \theta_{\mathrm{o}}=\theta_{\mathrm{o}}+\frac{{ }^{\theta_{\mathrm{o}}}{ }^{3}}{3}+\frac{2 \theta_{\mathrm{o}}{ }^{5}}{15}+\ldots \\
& \tan ^{2} \theta_{0}=\theta_{0}{ }^{2}+\frac{\theta_{0}{ }^{6}}{9}+\frac{4 \theta_{0}}{225}+\ldots \\
& +\frac{2 \theta^{4}}{3}+\frac{4 \theta^{6}}{15}+\frac{4 \theta^{8}}{45}+\ldots \\
& =\theta_{0}{ }^{2}+\frac{2 \theta_{0}{ }^{4}}{3}+\frac{17 \theta_{0}{ }^{6}}{45}+\ldots
\end{aligned}
$$


This is seen to be quite rapidly convergent, at least for small values of $\theta_{0}$, so that the magnitude of the first term dropped is still a good approximation to the error involved in dropping it and all following terms. Thus, for about $1 \%$ accuracy by this method, the range is estimated by setting

$$
\begin{gathered}
2 \theta_{\circ}^{4} \leq 0.01 \theta_{\circ}^{2} \\
3 \quad \leq \sqrt{0.015}=0.122 \mathrm{rad} \approx 7^{\circ}
\end{gathered}
$$

In order to relate the definition of effective solid angle, given at the beginning of this section, to the results of mapping the field as described in the preceding section (Section 3.4.2), let us consider the output from a radiometer with non-uniform directional response placed in a field of uniform radiance, $\mathrm{N}$, which completely fills both its aperture and its field. The value of $\mathrm{H}$ incident through an element of solid angle, $\mathrm{d} \boldsymbol{\Omega}$, in any given direction is, by definition, equal to $\mathrm{N} \cos \theta \mathrm{d} \Omega$. Hence the output may be computed from

$$
\begin{aligned}
\mathrm{V} & =\int_{0}^{2 \pi} \int_{0}^{\theta} \mathrm{N} \cos \theta \mathrm{R}_{\mathrm{H}}(\theta, \phi) \sin \theta \mathrm{d} \theta \mathrm{d} \phi \\
& =\mathrm{N} \int_{0}^{2 \pi} \int_{0}^{\theta} \mathrm{R}_{\mathrm{H}}(\theta, \phi) \sin \theta \cos \theta \mathrm{d} \theta \mathrm{d} \phi
\end{aligned}
$$

If this were an ideal radiometer with uniform response in all directions within a sharply defined solid angle, $\boldsymbol{\Omega}$, and with an irradiance responsivity equal to that of the actual radiometer in the direction of its maximum response, $\mathbf{R}_{\mathrm{H}, \max }$, then the output would be

$$
\mathrm{V}=\mathrm{HR} \mathrm{H}, \max _{\mathrm{N}}=\mathrm{N} \mathrm{R}_{\mathrm{H}, \max }
$$

As stated at the beginning of this section, the value of $\Omega$ which will make these two output values the same is called the effective solid angle, $\Omega_{\text {eff }}$. In other words, it is the solid angle of an equivalent ideal radiometer (uniform spatial response). If we equate Equations 27 and 28 , we obtain

$$
\Omega_{\text {eff }}=R_{H, \max }-1 \int_{0}^{2 \pi} \int_{0}^{\theta} R_{H}(\theta, \phi) \sin \theta \cos \theta \mathrm{d} \theta \mathrm{d} \phi
$$

For small values of $\theta$ we may approximate by using $\cos \theta \approx 1$ and $\sin \theta \approx \theta$, and write the approximate relation

$$
\boldsymbol{\Omega}_{\mathrm{eff}} \approx \mathrm{R}_{\mathrm{H}, \max }^{-1} \int_{0}^{2 \pi} \int_{0}^{\theta} \mathrm{R}_{\mathrm{H}}(\theta, \phi) \theta \mathrm{d} \theta \mathrm{d} \phi
$$

In terms of the coordinates, $\xi$ and $\eta$, defined in Figure 6 and Equation 8 , the effective solid angle is given by: 


$$
\boldsymbol{\Omega}_{\mathrm{eff}} \approx \frac{\mathrm{A}_{\text {eff }}}{\mathrm{s}^{2}}=\frac{\iint \mathrm{R}_{\mathrm{H}}(\xi, \eta) \mathrm{d} \xi \mathrm{d} \eta}{\mathrm{s}^{2} \mathrm{R}_{\mathrm{H}, \max }}
$$

where $A_{\text {eff }}$ represents the effective area of the image of the field stop and $s$ is the distance from the field stop image to the instrument aperture.

As noted in the WGIRB Report, Section 5.5, since this expression involves only the ratio $R_{H} / R_{H, m_{a x}}$, absolute measurements of the irradiance responsivity as a function of direction are not needed for this computation of effective field angle; relative values are sufficient. It is useful, as part of an actual calibration, to carry out this computation to compare it with the value obtained from separately determined values of the radiance responsivity and the irradiance responsivity in the direction of maximum response used in the expression (based on Equation 2)

$$
\Omega_{\text {eff }}=\mathrm{R}_{\mathrm{H}, \max } / \mathrm{R}_{\mathrm{N}}
$$

In particular, if the use of an extended source which fills a very much larger solid angle than the purely geometrical solid angle subtended by the field stop, results in a much smaller value of $\Omega_{\text {eff }}$ as computed from Equation 32 than that from Equation 30 or 31, one should look for the possibility that the instrument is responding to scattered radiation from large angles outside the field because of inadequate baffles or inadequate nonreflective coating of structural surfaces in the beam.

\subsection{TEMPORAL RESPONSIVITY ([ 8 and 9] Section 3.1)}

It is usually possible to design radiometers so that the frequency response (see footnote) is ample for the conditions of calibration and measurement. In that case, no detailed calibration is required. It is merely necessary to check the response time to verify that the instrument will respond rapidly enough to measure the desired changes in the incident radiation. This is easily done for most instruments which have $d-c$ response by rapidly opening and closing a shutter in front of a convenient source. Observations are then made of the time required for the output to reach its final value in response to the practically instantaneous change in the radiation incident on it.

It has already been pointed out that, in some specialized instruments which employ some form of scanning to translate spatial or spectral variations into temporal variations, the temporal or frequency response capability of the instrument may be the limiting factor in determining the corresponding spatial or spectral resolution. This will be discussed in more detail in later sections.

A basic radiometer may be used to measure changes in incident radiation, or to measure the modulation of the output of a source. The first type may have zero response at zero frequency in order to respond only to changes in radiation level (Section 4.3.5.2, Reference 9). Since the frequency-response characteristics are due to the combined characteristics of the detector element, the amplifier, and the output presentation components, much can be done with appropriately designed electronic circuits to modify and control this characteristic, at least within limits imposed by the detector element. 
One form of instrument is deliberately designed to respond to the time rate of change of the incident radiant power (i.e., to the first derivative with respect to time). However, there is always an upper limit, usually set by the frequency response capability of the detector element, which should be determined by calibration measurements.

If the radiometer is being calibrated for use in the measurement of modulation or fluctuation in the output of a target, it is essential that its responsivity as a function of frequency be established over the full range of frequencies that are to be measured.

3.5.1. TEMPORAL RESPONSIVITY CALIBRATION. Observation of response to a rapidly opened or closed shutter to determine that an instrument has an adequate response time has already been mentioned as a sufficient check in many cases. If somewhat more extended frequency response is needed to follow expected fluctuations in incident radiation, a check can be made by using a rotating mechanical shutter or chopper in front of a convenient source, with provisions both to vary and to measure the speed of rotation. If the upper limit of uniform frequency response is not critical and not difficult to obtain in terms of the fundamental frequency produced by the rotating shutter, the question of the waveform of the output of the detector element need not be raised. However, if the response does appear to fall off at frequencies below those to be measured and adjustments are not easily made in the electronics or elsewhere to correct this, it may be necessary to examine the waveform of the modulated radiation level produced by the shutter or chopper. The output can be recorded at a lower chopper rotation speed where the response is still linear, and can be analyzed mathematically to determine the relative strengths of the various harmonics in order to determine which are first being attenuated in the output as the speed of rotation of the chopper is gradually increased. However, another approach, which will avoid ambiguity and involved computations, is to use an aperture and chopper-blade configuration designed to produce almost purely sinusoidal chopping. Mechanical choppers can be designed to modulate a radiation beam over the range from zero frequency up to several hundred thousand cps, a range which is adequate for most measurements.

\subsection{REFERRAL OF MEASURED QUANTITIES TO THE SOURCE}

Up to this point we have discussed the response of radiometers in relation to the incident radiation at the reference location, just in front of the entrance aperture of the instrument. This has been stressed in order to emphasize that an instrument, when placed in a radiation field, necessarily responds to the conditions existing at that location. Moreover, it is possible to calibrate in terms of the radiometric field quantities existing at this reference location with assurance that measurement conditions are being reproduced. If the calibration results are related to more remote locations, less confidence can be placed in the calibration and the measurements based on it. When attempts are made to infer, from radiometric measurements, the properties of the radiation field at a distance from the radiometer, particularly in the neighborhood of the source emitting the radiation, it is found that the chances for error and ambiguity are substantially increased. Nevertheless, it is frequently the properties of a more or less distant source that are of primary interest. 
There are a number of factors to be considered. Sometimes it is difficult to obtain an accurate measurement of the location of the source, especially of a remote and rapidly moving source, and inferences about its radiant intensity from the irradiance at the radiometer depend upon the accuracy with which the distance is known. However, the two principal sources of difficulty are background sources and atmospheric attenuation, the latter usually being complicated by lack of information about the spectral distribution of source radiation.

3.6.1. BACKGROUND SOURCES. The distinction between a target source and a background source of radiation is an arbitrary one which depends only upon the interest of those making a measurement and is not an inherent property of the source. In calibration, any radiation which enters a radiometer along with the radiation from the desired source and which, hence, can cause an additional output response not correctly attributable to the source of interest is classed as background radiation.

As previously noted, background sources can be a particularly troublesome source of error in distant small-source calibration and in near small-source (Jones method) calibration. It should not be overlooked also, as suggested in the discussion of Section 3.4.3, that radiation from particularly strong sources which lie substantially outside of the geometrical field solid angle may still be scattered into the field stop due to inadequate optical design. Consequently, this type of background source radiation may also be troublesome in the extended-source calibration configurations.

Since calibration measurements are usually conducted under fairly well controlled conditions, it is often possible, with a little care, to eliminate most of the serious background sources. However, particularly when working in the longer infrared wavelengths where even objects at room temperature become substantial sources, one may find it impossible to eliminate all significant background sources. The only alternative, then, is to identify the desired source radiation by some distinctive modulation, so that the output response due to it can be differentiated from that due to the background. The simplest method is merely to turn it on and off with a shutter at the source and take the difference between output readings under these two conditions. Even then it may sometimes require considerable pains and ingenuity to insure that the shutter is not itself a source, either due to an elevated temperature resulting from radiation absorbed from the source which it is blocking, or as the result of reflections of radiation from unsuspected sources elsewhere. In fact, at the longer infrared wavelengths, it is probably impractical to attempt to devise a true shutter that will function as a non-radiating source, and the best solution is to deliberately design a second source of different, but equally well determined, value which can be substituted for the first one. Thus, the calibration becomes a measurement of the difference between the output indications from two known sources with all background conditions held constant. In these longer wavelength regions, beyond six or seven microns, where radiation from the surroundings at room temperature begins to become a problem, it is almost mandatory to use only blackbody sources for calibration. The value of the radiation from any other type of source 
becomes uncertain to the extent that it may include radiation reflected from the surroundings ([ 9], Section $5.1)$.

If a periodic interruption of the source radiation by means of a rotating chopper is employed, it is important to place the entire chopper (and any drive motor or other moving elements) behind a constant-temperature baffle which contains the limiting aperture defining the position and area of the source. (Any ray from any point in the radiometer aperture and within the radiometer field, which passes through this defining aperture, should intersect the source within the area and solid angle for which the radiation values are known.) Looking toward the source from the radiometer, the only portion of the chopper which should be visible is the portion of the chopper blade which passes in front of the source as seen through the defining aperture. With this arrangement, the defining aperture becomes alternately a source of radiance equal to that of the source behind it and then a source of radiance equal to that of the chopper blade.

3.6.2. ATMOSPHERIC ATTENUATION-APPARENT SOURCE RADIATION. The attenuation of infrared radiation by the atmosphere is a highly variable function of wavelength, and of meteorological parameters (particularly of the concentration of water vapor) which are themselves highly variable and not amenable to exact determination. Being a function of the wavelength, the net attenuation of a beam which is composed of a distribution of wavelengths can be estimated only when the spectral distribution of the beam is known. Thus, in order to assess the atmospheric attenuation of radiation in a spectral band from a distant source we must know the spectral distribution of the source radiation. Atmospheric spectral attenuation is so highly variable with wavelength that this statement is usually true, even for fairly narrow wavelength bands, such as the spectral slit width (see Section 6, below) of a spectrometer or spectroradiometer. In other words, before we can compute the atmospheric attenuation in order to arrive at the value of spectral radiance, $\mathrm{N}_{\lambda}$, or of spectral radiant intensity, $\mathrm{J}_{\lambda}$, at a distant source, we must know its relative spectral distribution, $r(\lambda)$.

For the reasons outlined in the preceding paragraph, there are unavoidable uncertainties involved in infrared measurements of a distant source in an attenuating medium, and particularly in the earth's atmosphere. Also, there is a lack of complete agreement on the best methods for dealing with this difficult situation. It is obvious, however, that it is desirable to report in as much detail as possible regarding the pertinent conditions of any measurement or calibration. These include the geometry; the meteorological conditions along all ray paths, including temperature, pressure (altitude), and humidity; and any indications of haze or scattering particle content. There is particular need for study and development of techniques for dealing practically with attenuation by scattering.

When source measurements are made over short paths in the laboratory, it is best to attempt to eliminate the effects of atmospheric attenuation by flushing out the optical paths with a gas such as dry nitrogen. 
Failing this, one should make calibration measurements which reproduce as nearly as possible all of the measurement conditions.

As discussed in Section 2.3.2, the second technique has been profitably employed during field measurements of many targets such as armored vehicles and buildings. In the case of other targets no possibility exists for calibration in this manner. At best an arrangement may be devised to allow direct evaluation of a attenuation by the intervening atmosphere. Often data are reported with no adjustment for atmospheric effects; this practice, which has been discussed in Reference 8 and the WGIRB Report, involves the use of the modifier "apparent" for quantities which describe the source which would produce the same radiation at the instrument aperture if no intervening atmosphere were present. The following notation follows that of Reference 8 and is a modification of that recommended by the WGIRB Report for such cases:

$$
\mathrm{J}^{\prime}=\tau \mathrm{J}=\mathrm{HS}^{2} \text { and } \mathrm{N}^{\prime}=\tau \mathrm{N}
$$

where $J^{\prime}=$ apparent radiant intensity

$\mathrm{N}^{\prime}=$ apparent radiance

$\tau=$ atmospheric transmittance (for the particular path and spectral beam)

$\mathrm{H}=$ irradiance at radiometer due only to source radiation

$\mathrm{N}=$ radiance at the source

$\mathrm{J}=$ radiant intensity of source

$\mathrm{S}$ = distance from radiometer to source

In (33) all of the radiometric quantities refer only to radiation in the beam from the source since radiation from background sources has been ignored. The same relations hold if the spectral quantities, $J_{\lambda}^{\prime}, \tau(\lambda)$, $\mathrm{J}_{\lambda}, \mathrm{H}_{\lambda}, \mathrm{N}_{\lambda}^{\prime}$, and $\mathrm{N}_{\lambda}$, are used.

When a calibration is being made in support of measurements which are to be reported only in terms of the apparent radiometric quantities for distant sources, the calibrations should be made as nearly as possible in terms of the radiometric quantities at the reference position. Thus the reported measurement values can be regarded as absolute values at least at the reference position. The uncertainty is limited only to that resulting from atmospheric attenuation conditions for the measurement of the distant source. If, however, the calibration results are also determined only in terms of the apparent radiance or apparent radiant intensity of the known source, as observed by the radiometer through an unknown atmosphere, their use introduces a second uncertainty into an already uncertain situation. The only exception is the one already suggested - when it is really possible to reproduce all of the conditions for both the calibration and the subsequent measurement, so that, in effect, the unknown source is being directly compared with the calibration source under exactly corresponding conditions.

If computations of atmospheric attenuation are needed to obtain the values of the radiometric quantities at the reference position of a calibration measurement, full details of the computations and the meteorological 
data on which they are based should be included in the calibration report.

3.6.3. APPARENT SOURCE TEMPERATURE VS. APPARENT SOURCE RADIATION. Just as the reference radiation is sometimes conveniently specified in terms of a reference temperature, so in many instances radiance has come to be expressed by temperature units, particularly in connection with lower temperature targets such as vehicles and terrain features. As pointed out in the WGIRB Report, Section 4.3.2, the source temperature uniquely describes its radiance only if the source is a blackbody. For graybodies a combination of temperature and total emissivity is required. For other situations, involving spectral emissivity variations and non-equilibrium conditions with temperature gradients, it becomes quite meaningless to ascribe a temperature to the source on the basis of the apparent radiance observed with a radiometer. This is particularly true if the radiometer has a spectral characteristic as well. It is strongly recommended that the possible confusion that may result from this use of temperature be avoided and that the results be expressed, instead, in units of apparent radiance supplemented by a statement of the spectral characteristic of the instrument and the method of normalization used by given (see Section 3.3.2, above). Accordingly, we do not recommend calibrations in units of source temperature.

\subsection{LINEARITY}

It was assumed in the ideal case (Section 2.1) that the output of a radiometer is directly proportional to the incident radiant power. This is implied also by the relations (1) defining the responsivities, which are usually considered to be constants. However, the responsivity of a radiometer need not be a constant. In some instances, in order to accommodate a greater dynamic range, and at the same time to maintain an approximately constant percentage of precision over all parts of that range, instruments are deliberately designed to have an output which, after some arbitrary level is reached, is proportional to the logarithm of the incident radiant power. In such a case, the establishment of the reference radiation level for the zero point of the output scale (see Section 2.3.7) becomes even more important than for an instrument with linear response, as previously discussed.

It is particularly difficult to generalize about this matter of nonlinearity because, as with the other features of radiometers, there are many subtle variations. Probably the best general comment is that non-linearity in any system should be treated as a warning of potential difficulty and should be carefully analyzed to determine the possibility that it may distort the input-output relations in unintended ways. For example, consider a radiometer with a chopper designed for background discrimination. The electrical signal at the output of the detecting element (input to the amplifiers) is usually proportional to the difference between the radiant power in the beam of radiation from the source to be measured (and possibly also some of the background, which we shall ignore for the moment) and the radiant power in a reference beam of radiation from adjacent portions of the background. In most cases, the amplitude of the signal out of the detector element 
is directly proportional to this difference in radiant power over a wide dynamic range. Thus, regardless of the subsequent use of a logarithmic or other non-linear amplifier, the final output signal can be calibrated, without ambiguity, as a measure of this difference in the incident radiant power. However, if the source is quite small and far away, so that its image is concentrated in a very small area of the surface of a detector at the field stop, and if the radiant power concentrated in this small image at times exceeds the irradiance to which the detector will respond linearly, local saturation takes place. In this case the electrical signal from the detector itself is no longer directly proportional to the difference in incident radiant power. There is no information in the final output signal to show when this type of saturation is taking place, so the interpretation in terms of incident target radiation becomes ambiguous. This, incidentally, is another strong reason for the use of a field lens (see Section 3.4.1).

We have said that a complete set of calibration measurements should reproduce as far as possible the full range of values for the different quantities in situations such as those discussed in the preceding paragraph. Thus, one should try to make calibration measurements over a range of irradiance at the entrance aperture of the instrument-a range which includes the highest values expected in actual measurements. Similarly, sources should be used which are imaged through the smallest field-angle element expected for a source in any of the measurements based on this calibration. The way in which the responsivity varies as a function of these variables should be observed in order to determine if, and at what point, any nonlinearities may occur. The analysis of such situations must be thorough and painstaking to insure that all relevant factors which may arise in the measurement situation have been evaluated.

On the other hand, it must be recognized that there may be some basic limitations making it impossible to achieve the entire recommended range of calibration checks. The Planck radiation law establishes a definite limit to the maximum radiance that can be obtained from any thermal source at a given temperature, and there are limits to the temperature that can be achieved in a practical calibration source. Furthermore, the invariance of radiance along any ray makes it impossible to increase the radiant power from a source of given radiance through a given aperture and field angle, once both are completely filled, regardless of changes in external configuration or the introduction of any additional external optical elements.

3.7.1. LINEARITY CALIBRATION. The linearity of response (constancy of responsivity as a function of input level) can be measured by use of a number of methods for obtaining different known levels of incident radiation at the reference position (radiometer entrance aperture). Each method has advantages and disadvantages.

Probably the most frequently used method, at least in both of the small-source configurations, is merely to change the size of the source. Commercially available blackbody sources are often supplied with an aperture plate with multiple apertures, making it possible to change the aperture area quickly by known amounts. Although this is certainly convenient, it has the disadvantage that, in the distant small-source configuration 
(see Section 2.3.1), it also changes the size of the source image in the image plane of the radiometer. If the detector is located in the image plane, this means that there is a simultaneous change both in the total amount of radiant power and in its distribution over the surface of the detector. It thus becomes quite complicated to relate any change in responsivity unambiguously to one or the other of these changes. In the near smallsource or Jones method configuration care must be exercised to insure that, even with the largest aperture used, the limitations on source size and position are maintained as described in Section 2.3.4. Also, changes in source size produce corresponding changes in the region of the aperture through which radiation passes to the field stop (see Figure 5). The output can also be affected by variations in spatial responsivity across the aperture. However, as discussed in Section 3.4, this configuration should not be used unless checks have established that such spatial variations do not exist to a significant degree or unless enough measurements are made with a systematic distribution of source positions in relation to the aperture to insure that an average of the results is representative of the response to uniform irradiation of the full aperture.

Another common method of obtaining a distribution of points along the responsivity curve is to vary the temperature of a blackbody calibration source - a method used particularly with extended-source configurations. It has the disadvantage of inconvenience-blackbody sources must arrive at temperature equilibrium before they provide a reliable level of output radiance. After this they cool very slowly; so it takes a substantial amount of time to make measurements over any range of temperature values. Even more serious, huwever, is the fact that the spectral distribution of radiance is changing simultaneously with the total radiance. This means that the computation of the normalized radiance based on the relative spectral responsivity of a selective radiometer requires a separate integration for each value of temperature. This adds considerably to the labor and to the opportunity for error. Furthermore, it does not provide a check of linearity, which is clearly independent of other parameters.

Accurately known irradiance values, readily computed by the inverse-square relationship, can be obtained by moving a small source on an optical bench carriage. This will not change the spectral distribution if we can neglect the variation in the amount of atmosphere which is traversed by the radiation. However, this motion will change the size of the source image; and even worse, it will be accompanied by motion of the image plane, necessitating refocusing of the radiometer for each measurement. The focusing arrangements may themselves affect the responsivity of the instrument. Slight changes in alignment of the optical elements can interact with the optical aberrations affecting, for instance, the minimum image size and the directional response. Movement of the source can also shift the position of its image in the image plane, requiring reorientation of the radiometer for the direction of maximum response. If, however, a good field lens is used (i.e., exploration of the field shows uniform directional response over a well-defined field), then these effects will not be a source of difficulty as long as precautions are taken to insure that all source rays passing through the receiving aperture at its nearest position, are still well within the field stop. 
In using receivers and sources of finite areas on an optical bench, it is important to recognize the limits to the validity of the inverse-square relationship when the distances used are measured along the bench between source and receiver. The inverse-square law applies strictly between any pair of infinitesimal areas of the source and of the receiving aperture, using the slant distance between them. However, the integrated result for all such pairs is only approximately an inverse-square variation of the total flux through the receiving aperture from the entire source in terms of the distance between their centers (as measured along an optical bench with both source and receiver perpendicular to the bench and centered at the same height above it). Walsh [ 11] has computed the integrated values of irradiance at finite distances from a disc along the line perpendicular to the source through its center, as well as the deviation from the inverse-square relationship. Graphically shown, his results indicate that the relation is accurate to within $1 \%$ at distances greater than ten times the radius of a circular disc source. However, this is only at a receiver point "on axis." When the receiver, too, is of finite dimensions, the situation becomes somewhat more complicated. A common working rule in using an optical bench is not to apply the inverse-square relationship to distances less than twenty times the largest dimension of source or receiver. However, such a rule should not be used blindly. It must be realized that the number chosen depends entirely on the degree of precision desired, and that the choice of a factor of twenty represents a precision of the order of a few percent.

Still another method, which can be used most easily with a collimator (see Section 2.3.6), is to obtain variations in incident radiant power at the radiometer aperture by inserting precision screens or gray filters which cut out fractions of the collimated beam. The reduction can be determined accurately by measurements of the clear and opaque areas of the screens as long as diffraction effects are negligible. This has the disadvantage that, in effect, it modifies the area of the radiometer aperture stop through which radiation passes to the field stop. Consequently, it should be used with caution in cases where there is any question about variations in spatial responsivity across the radiometer aperture stop. This is true particularly when a field lens is used to image that aperture stop on the surface of a detector which may have variations in responsivity over its sensitive surface. In such cases, however, it may be possible to rotate the screens and take the average output as the response which would be obtained to uniform irradiance of the entire aperture at the average value.

\subsection{DETECTOR TEMPERATURE}

Unfortunately, the responsivity of many detector elements is a function of the temperature of the element. This makes it highly important that this temperature be controlled or, if it cannot be controlled, that it be measured and recorded. The effects of detector temperature and the effectiveness of the provisions for controlling it should be checked as part of any complete calibration. If the temperature is uncontrolled, then calibration measurements should be carried out at enough different detector temperatures so that a record of the temperature at the time of a measurement will establish the proper value of instrument responsivity 
to be used in evaluating the instrument output for that measurement. While we do not know what the effects of a temperature gradient in a detector element may be, it should be noted that such gradients may exist, particularly in the so-called bulk detectors and that this may significantly affect the responsivity.

4

\section{CALIBRATION OF A CHOPPER RADIOMETER}

Although it is often overlooked, one of the main reasons for employing a chopper in a radiometric instrument is to get away from the drift problems that can be so troublesome in d-c instruments. Amplifier design is also facilitated by producing an a-c rather than a d-c output from the detecting element. (a-c amplifiers are, in general, simpler to design than equally stable d-c amplifiers.) The reference radiation of the instrument is established in terms of the radiation from the chopper blades. Furthermore, in some difficult field situations, choppers can be designed to provide background discrimination. This may make it possible to obtain measurements in situations where a radiometer that responds to the total radiant power incident within its field would be more easily saturated by a high level of background radiance. Because of the frequency with which chopper radiometers are employed and the large number of variants among them, it seems desirable to accord them special treatment in this separate section.

There are two important ways in which the use of a chopper in a radiometer can affect the calibration. One is the effect on the reference radiation or zero point of the output scale. The other is through what we have termed waveform problems. This refers to the ways in which the waveform of the output signal of the detector element and, ultimately, the final output may depend on the interaction between the chopper configuration and the size, orientation, and position of the image of the source in the field stop of the radiometer. In turn, the shape of the resulting signal waveform can affect the responsivity.

\subsection{CHOPPER AS SOURCE OF REFERENCE RADIATION ([ 9], Section 4.3.1)}

As already stated, one of the principal reasons for using a chopper is the stability which it can provide by establishing the reference radiation level. At short wavelengths, through the UV and visible out to about one micron in the near IR, this is accomplished rather easily merely by making sure that the chopper surface is uniformly coated with a black surface. Even through the lead sulfide region, out to about $3 \mu$, the temperature of the chopper usually is not a critical factor because at ambient temperatures there is little radiation in these shorter wavelengths from a black or gray body. However, in the case of detectors which respond to longer wavelengths, the effects of variations in chopper temperature and emissivity become a serious consideration. Emissivity effects can be minimized by using a highly reflecting chopper blade, but then it is important to control the incident radiation reflected from the surroundings to the detector by the 
polished chopper. This is done in many radiometers by mounting a small reference blackbody in a position where, by specular reflection from the chopper-mirror, radiation from this reference source completely replaces the external radiation beam when the latter is blocked by the chopper. This not only provides a stable source of reference radiation, but also an adjustable one when appropriate temperature controls are provided for setting the reference blackbody temperature at different values. As defined in Section 2.3.7, the reference radiation value will not necessarily agree with the actual radiance of this reference blackbody. Discrepancies may be due to differences in the solid angle through which this reference source irradiates the detector element as compared to the solid angle for external radiation. Other discrepancies may originate in the number of reflections in the optical paths traversed by the reference radiation and that from external sources, or in possible partial polarization of the radiation. It is important, as part of the calibration of such an instrument, to measure and establish the reference radiation level, as well as the responsivity, by means of external calibration sources.

4.1.1. LOCATION OF CHOPPER. It is desirable that the chopper establish the reference level with respect to the beam of radiation from the source, uncontaminated as far as possible by background radiation. Thus the ideal location for a chopper is immediately in front of the emitting source and behind a baffle plate. This defines the beam from the source to the radiometer as described at the end of Section 3.6.1 above. It is not always feasible to place it there, however, for obvious reasons. For convenience it is usually desired that the chopper be incorporated as a part of the radiometer, particularly if the instrument is to be portable and to be used under varying circumstances to measure a variety of sources.

When the chopper is part of the instrument, the foregoing considerations make the preferred location for it just in front of one of the stops, either the aperture stop or the field stop. With this arrangement, radiation reaches the detector alternately from the external beam and from the chopper through the stop. Radiation reaching the detector from the surroundings outside of the stop opening, including that from the material which defines the stop, is not modulated by the chopper. Any filters, particularly if they might radiate significantly because of their own temperatures, should also be placed between the chopper and the detector where any such self-radiation will not be chopped or modulated. The location in front of the aperture stop is seldom used. The field stop is usually much smaller than the aperture stop so that a smaller chopper will suffice at the field stop location.

Unfortunately, there are still other considerations, some of them conflicting, which also have a bearing on the chopper location. A field stop must be positioned accurately in the image plane of the collecting optics if the field of view is to be sharply defined. However, when a chopper is being used for background suppression, it is important that it too be located in the image plane, as will be discussed in Section 4.2. This can be accomplished by using additional optics to reimage the image plane in a second image plane. The field stop is located in one and the chopper in the other. This arrangement adds to the size and com- 
plexity of the instrument and the optical losses are increased by the additional optical element(s), so it is not frequently employed. On the other hand, if it is desired to measure only the radiant power from a target, regardless of its size, shape, and location in the field, and to measure it within as wide limits as possible (which can only be done with minimum background suppression), the chopper definitely should not be close to the field stop and, ideally, should be just in front of the aperture stop. These considerations are reviewed here because of their bearing on calibration measurements. It is important in making calibration measurements to know and evaluate all of the parameters which may affect responsivity.

\subsection{WAVEFORM PROBLEMS}

Whenever a chopper is located in, or close to, the image plane of a radiometer, for the reasons given in the preceding section, the waveform of the modulated output of the detection element becomes a function of the target image. The waveform is affected by the image size, shape, orientation, and location in the field stop. How these parameters, in turn, affect the final output, and hence the responsivity, depends greatly on the subsequent signal processing by the amplifiers and the presentation device. Such things as the frequency bandwidths and the type of demodulation used, particularly phase-sensitive demodulation, may make a great deal of difference in the way in which responsivity varies with these parameters of the target image. While it is possible to predict these effects by sufficiently thorough analysis, the best course is to verify them by appropriate calibration measurements. This can be done with a known source for which these different parameters can be varied independently in a controlled manner. Probably the use of a collimator, as described in Section 2.3.6, is the most practical approach. The parameters of the source image can be controlled by varying the size, shape, and orientation of an aperture located at the focus of the collimator. The location of the source image in the image plane of the radiometer is shifted by rotation of the collimator about the center of the radiometer aperture described in Section 3.4.2 (see Figure 8).

5

\section{CALIBRATION OF SPECTRORADIOMETERS}

We consider spectroradiometers to be those instruments which can be used to obtain an absolute measurement of the spectral variation of a source radiometric quantity. These instruments can use prisms, diffraction gratings, and many other optical elements to produce spectra. In a sense, radiometers which rapidly sequence through a set of narrow filters also can be called spectroradiometers. Since the previous discussion is directly applicable to such instruments, they will not be treated in this section beyond the mention on one precaution to the reader. Often the filter sequencing is accomplished by the rotation of a wheel containing the filters. If the speed of rotation is sufficiently great, the dwell time for any one filter can approach the response time of the unfiltered radiometer. In such cases, the response time should be deter- 
mined under dynamic rather than static conditions.

The spectral measuring instruments have one property in common. The various wavelengths present in the source radiation are spread or dispersed in space. Prisms make use of the variation in index of refraction with wavelength to reveal the radiation spectral content. Diffraction gratings can disperse a parallel bundle of rays into sets of angles corresponding to the associated order numbers. Fresnel zone plates can disperse wavelengths along their optic axes. The fringes obtained with interferometers will be located in different portions of space according to the wavelengths present in the radiation field.

If the absolute magnitude of the radiation at the dispersed wavelengths is measured, then a spectroradiometric determination has been made. The calibration of such instruments must encompass the establishment of both a wavelength scale and a radiometric scale. In many cases these scales are not independent, so that the calibration involves considerably more than establishing the effects of varying each parameter separately.

The need for complete familiarity with the characteristics and operation of the instrument is even greater for dispersing spectroradiometers than for the less sophisticated types of radiometers discussed previously. The need is greater because of their added complexity and the increased opportunity for ambiguity and error. It is not feasible to include here a complete discussion of all of the subtleties of dispersing instruments. The reader is urged to consult, and study thoroughly, good standard texts on this subject, such as References 12 and 13, as well as the articles and books listed in the bibliography and on p. 401 of Reference 8. All that will be included here will be comments about some of the most important features which affect the calibration of these instruments for quantitative spectroradiometric measurements.

We have already stated, and emphasized as a general principle, that calibration measurements should be made under circumstances which reproduce, as completely as possible, all of the conditions which will apply during the measurements based on those calibrations. In connection with the use of dispersing instraments, this statement needs even stronger reemphasis. The possible interactions, especially if non-linearities exist, between the various components of an actual monochromator or spectrometer, can be extremely perplexing. Without verification based on extensive experience with the particular instrument, no attempt should be made to compute the results obtained with one combination of variables (such as slit widths, scan speeds, and gain settings) from calibration measurements made with a different combination of these parameters. A separate calibration should be made with each combination that will be used in actual measurements. With this caution about combined net effects and interactions, we will proceed to consider individually the features and their effects for several instruments. 
A particularly common form of spectroradiometer is one which uses prisms and/or gratings with an entrance slit and one or more exit slits. This type of instrument is treated in some detail in the following section. In view of the tremendous versatility of interferometers, a brief discussion on a few pertinent interferometer characteristics is given in Section 5.2 .

\subsection{PRISM AND GRATING INSTRUMENTS}

The essential components of any prism or grating spectroradiometer are the same as those of any radiometer, with the addition of the following (see Figure 11):

(a) An entrance slit, which usually acts as the field stop of the collecting optics

(b) A collimator, which may be a lens or a mirror, with the entrance slit at its focus

(c) A dispersing element, either a prism or a grating, which spreads the beam from the collimator into a dispersion of parallel beams, each wavelength continuing in a collimated beam at a slightly different angle than that formed by other wavelengths

(d) A focussing element, which produces an image of the entrance slit from the parallel beam at each wavelength so that these images are dispersed linearly to form the familiar spectrum

(e) One or more exit slits to select the radiation in any desired region of the spectrum and allow it to pass on to the detector

Briefly, the foregoing can be summarized by saying that a spectroradiometer is a radiometer with a monochromator interposed between the collecting optics and the detector.

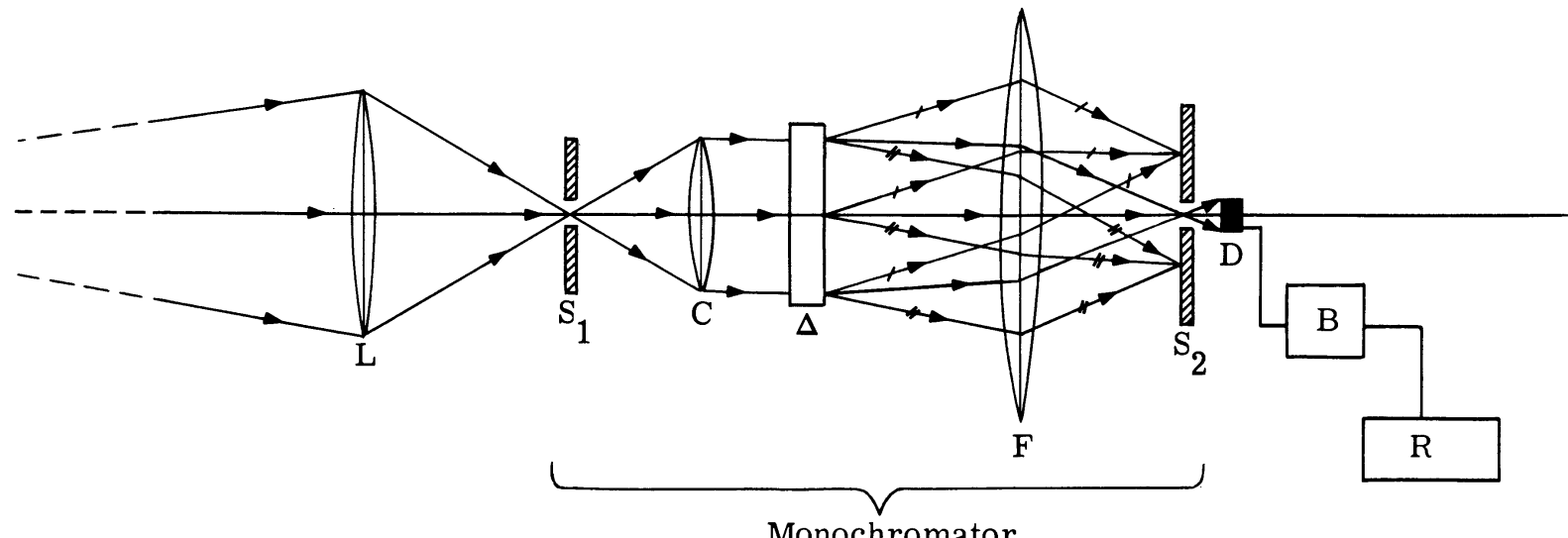

Monochromator

$\mathrm{L}=$ Collecting optics

$\mathrm{S}_{1}=$ Entrance slit of monochromator acting also as the field stop

$\mathrm{C}=$ Collimating optics

$\Delta=$ Dispersing element (prism or grating)

$\mathrm{F}=$ Refocussing optics
$\mathrm{S}_{2}=$ Exit slit of monochromator

$\mathrm{D}=$ Detector element

$\mathrm{B}=$ Amplifier and electronic circuits

$\mathrm{R}=$ Recorder for output presentation

FIGURE 11. A SIMPLE FORM OF SPECTRORADIOMETER 
In many so-called self-collimating spectrometers and monochromators, a single optical element serves both for collimating the beam from the entrance slit and for refocussing the dispersed beam onto the plane of the exit slit. One of the most frequently used configurations of self-collimating instruments is the Littrow type. In this instrument a plane mirror is positioned so as to return the dispersed beam back to the prism or grating for a second dispersion before it returns to the self-collimating mirror or lens and the exit slit.

In the so-called double-pass monochromators, the focused beam is sent back through the entire system a second time for still higher dispersion. If the double-pass instrument is a Littrow type, the beam is acted upon four times by the prism or grating. Not only is dispersion increased by these repeated dispersions but spectral resolution and purity are also improved. In a double monochromator (two monochromators in series, the exit slit of one forming the entrance slit of the second) the repeated dispersion also improves the spectral resolution and purity. The emerging beam from the exit slit of a well-designed double monochromator contains a minimum of scattered radiation of wavelengths outside the desired pass band.

In double-pass instruments, however, the second pass is made over portions of the same path as the first. In one type it is distinguished from first-pass radiation, also emerging from the exit slit, only by the insertion of a chopper in a portion of the second-pass beam that does not overlap the first. In this case, although the modulated beam from the second pass may have high spectral purity, there can be a fairly high level of unchopped first-pass radiation of unwanted wavelengths also incident on the detector. The effect of this first-pass radiation on the response of the detector element to the chopped second-pass radiation of the desired wavelengths should be carefully tested in such cases. This is particularly important for measurements in wavelength regions in which the spectral responsivity of the detector element can be very low as compared to its responsivity to the unchopped wavelengths from the first pass. Spectral filters inserted at the entrance slit can be used to produce substantial changes in the level of this unwanted, unmodulated radiation in order to observe the effect, if any, on the output. Ideally, there should be no effect, because the output should be a measure only of the chopped radiation in the desired wavelength band. However, this needs to be verified for each detector element used over the complete range of wavelengths for which it will be employed.

The wavelength band in the output of a prism monochromator is an unambiguous function of the relative positions of the slits, the prism, and any optical elements (such as a Littrow mirror) used to shift the dispersed spectrum across the exit slit. In a grating monochromator, however, there can be overlapping orders. Radiation of one wavelength diffracted in the first order will leave the grating parallel to that of another wavelength in another order, and the images in the spectrum can overlap. A fairly low-dispersion foreprism monochromator, or suitable filters, can be used in front of the entrance slit to remove wavelengths of the undesired orders and to eliminate the ambiguities. 
The wavelength assigned to an output from a dispersing instrument is defined as the wavelength of the ray from the center of the entrance slit to the center of the exit slit. In a spectrally scanning instrument this wavelength is changed by changing the position of any of the components which determine the relationship between the exit slit and the spectrum focussed in the plane of the exit slit. This can be done manually for a point-by-point scan, or mechanically for a continuous scan. In all cases, this wavelength of the ray between the slit centers will be called the instantaneous wavelength setting of the instrument.

There are also instruments with multiple exit slits with a separate detector associated with each slit. Here, too, we will refer to the wavelength of the central ray at each exit slit, as described above, as the wavelength setting for that slit-detector combination and hence the wavelength associated with that output. The problem of determining the spectral passband, spectral resolution, or spectral slitwidth at each wavelength setting will be discussed under the heading of slits and slit effects.

The wavelength calibration (i.e., the establishment of the scale of wavelength settings or the wavelengths to be associated with each output), is usually covered adequately in the instructions furnished with any commercially available instrument, as well as in the standard texts on spectroscopy. Accordingly, it will not be discussed here in any detail. Essentially, it consists of observing the output as a function of the wavelength setting when the entrance slit is irradiated by sources with well known, sharply varying spectra. These may include sources which emit sharp spectral lines, such as the familiar mercury green line and the yellow sodium D-lines in the visible, as well as continuous sources combined with filter materials such as polyethelene, polystyrene or various gases [14].

Our concern here will be to discuss more thoroughly the radiometric calibration by which values are assigned to the amplitude of the output associated with each wavelength setting. We will discuss, particularly, the ways in which this radiometric calibration is affected by the features peculiar to dispersing instruments. In addition, all of the considerations already treated in connection with less sophisticated radiometric instruments, such as the properties of the collecting optics, filters, choppers, detector elements, amplifiers, and output presentation devices, apply equally well to those components when used in a spectroradiometer.

The instrument responsivity can be determined at one wavelength (actually over a narrow wavelength region) by the use of a sufficiently narrow spectral line source for which the absolute spectroradiometric values are known. Such sources are especially useful in shorter wavelength regions in which the radiation from a blackbody is very often not of sufficient magnitude (over the wavelength interval corresponding to the spectral slit width) to insure a high signal-to-noise ratio. The source-receiver configurations discussed in Section 2.3 are appropriate for responsivity determinations for prism and grating instruments, although quite often the source is placed to illuminate uniformly a stop which comes after the entrance aperture stop. 
The spectral responsivity of a spectroradiometer must be specified both as a function of the spectral slit width and of the instantaneous wavelength setting. The first wavelength dependency is a static function, while the second is a dynamic one developed by the wavelength scanning. It should be noted that these are not independent quantities. The spectral slit function can be considerably different at different wavelength settings. This subject is adequately treated in the literature referenced before, but a brief review will be useful.

The spectral.slit width, $\Delta \lambda_{s}$, at an instantaneous wavelength setting, $\lambda_{i}$, for slit widths sufficiently large that diffraction effects are negligible (this is most often the case for instruments used in field measurements of sources) is indicated in Figure 12 for equal entrance and exit slits, and for linear dispersion.

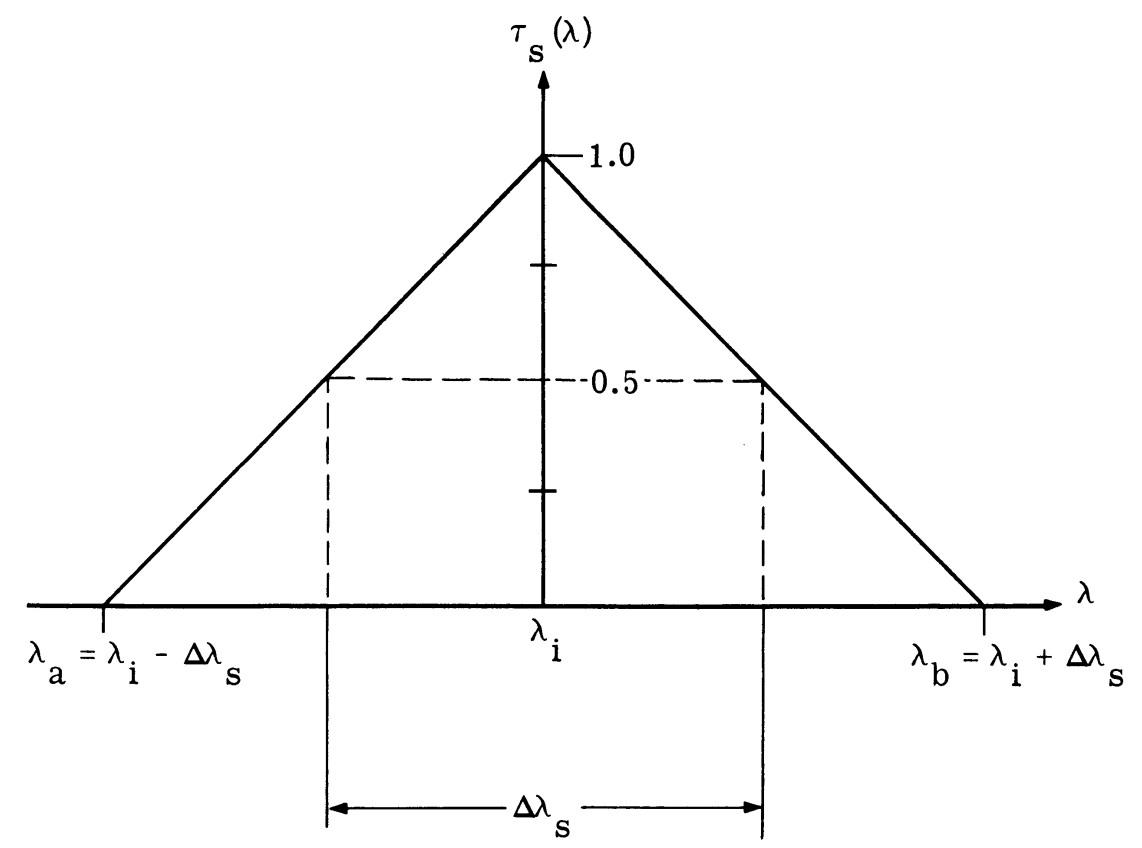

FIGURE 12. TRIANGULAR SPECTRAL SLIT WIDTH

Thus, $\Delta \lambda_{S}$ is the half intensity breadth, and the transmittance, $\tau_{S}(\lambda)$, is represented by

$$
\begin{aligned}
& \tau_{s}(\lambda)=\left(1-\frac{\left|\lambda_{i}-\lambda\right|}{\Delta \lambda_{S}}\right) \text { for } \lambda_{i}-\Delta \lambda_{S} \leq \lambda \leq \lambda_{i}+\Delta \lambda_{S} \\
& \tau_{S}(\lambda)=0 \text { for all other values of } \lambda
\end{aligned}
$$

If the slits are of unequal width, then under our idealizing assumptions, the spectral bandwidth is as shown in Figure 13. Now the instrumental half-intensity breadth, $\Delta \lambda_{\mathrm{S}}$, is given by $\Delta \lambda_{\mathrm{w}}$, the spectral slit width 
corresponding to the wider slit. Thus we have:

$$
\begin{aligned}
& \tau_{\mathrm{s}}(\lambda)=0 \text { for } \lambda \leq \lambda_{\mathrm{a}} \\
& \left.\tau_{\mathrm{s}}(\lambda)=\frac{\lambda_{\mathrm{a}}-\lambda}{\Delta \lambda_{\mathrm{n}}} \text { for } \lambda_{\mathrm{a}} \leq \boldsymbol{\lambda}_{\mathrm{a}} \boldsymbol{\lambda}_{\mathrm{a}} \Delta_{\mathrm{n}}\right) \\
& \tau_{\mathrm{s}}(\lambda)=1 \text { for }\left(\lambda_{\mathrm{a}}+\Delta \lambda_{\mathrm{n}}\right) \leq \lambda \leq\left(\lambda_{\mathrm{b}}-\Delta \lambda_{\mathrm{n}}\right) \\
& \tau_{\mathrm{s}}(\lambda)=\frac{\lambda_{\mathrm{b}}-\lambda}{\Delta \lambda_{\mathrm{n}}} \text { for }\left(\lambda_{\mathrm{b}}-\Delta \lambda_{\mathrm{n}}\right) \leq \lambda \leq \lambda_{\mathrm{b}} \\
& \tau_{\mathrm{s}}(\lambda)=0 \text { for } \lambda \geq \lambda_{\mathrm{b}}
\end{aligned}
$$

The spectral power responsivity of the detector, normalized to the peak of its response, can be used to obtain the output by the following relation:

$$
\mathrm{V}\left(\lambda_{i}, \Delta \lambda_{s}\right)=\mathrm{R}_{\mathrm{D}}\left(\lambda_{\mathrm{m}}\right) \int_{\lambda_{\mathrm{a}}}^{\lambda_{\mathrm{b}}^{\mathrm{b}}} \cdot \mathrm{P}_{\lambda}(\lambda) \tau_{\mathrm{s}}(\lambda) \mathrm{s}(\lambda) \mathrm{d} \lambda
$$

where $V\left(\lambda_{i}, \Delta \lambda_{S}\right)$ is the spectroradiometer output at wavelength setting $\lambda_{i}$ and with a spectral instrument width $\Delta \lambda_{S}$

$R_{D}\left(\lambda_{m}\right)$ is the spectral power responsivity of the detector, normalized to the peak value at $\lambda_{m}$

$\mathrm{P}_{\lambda}(\lambda) \quad$ is the spectral radiant power at the entrance slit. (It is assumed that the collecting optics have been carefully matched to the optics of the monochromator so that the aperture stop of the collector serves as the aperture stop of the combined system and there is no subsequent vignetting, e.g. by any of the optical components of the monochromator.)

$s(\lambda)$ is a function encompassing the relative spectral responsivity of the detector and all other spectrally dependent quantities such as the reflectance and absorptance of the mirror surfaces, prisms, etc.

$\tau_{S}(\lambda)$ is the idealized spectral transmittance.

If we were to write Equation 36 in terms of the spectral power responsivity of the instrument, then the quantities $\mathrm{s}(\lambda), \tau_{\mathrm{s}}(\lambda)$ and $\mathrm{R}_{\mathrm{D}}\left(\lambda_{\mathrm{m}}\right)$ would appear lumped together in the quantity $\mathrm{R}_{\mathrm{P}_{\lambda}}(\lambda)$.

In the case of narrow slits the spectral transmittance may take on the shape of a typical single slit diffraction pattern. In this case,

$$
\tau_{\mathrm{S}}(\lambda) \sim \frac{\sin ^{2} \beta}{\beta^{2}}
$$




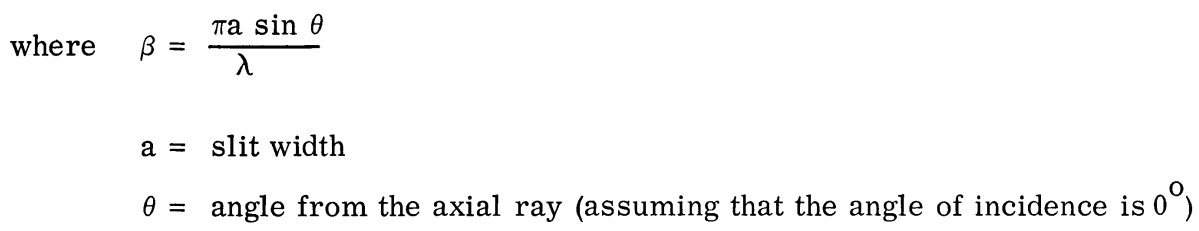

The use of Equation 37 in 36 is then necessary.

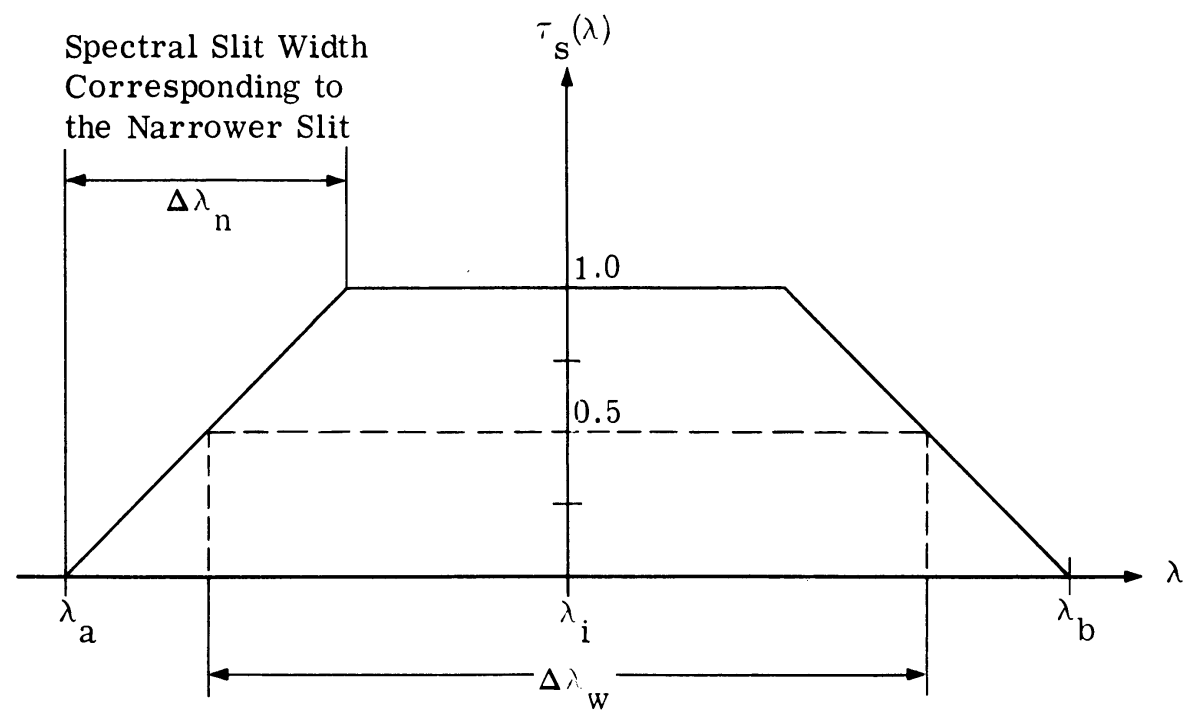

Spectral Slit Width of Wider Slit

$$
\begin{aligned}
& \lambda_{\mathrm{a}}=\lambda_{\mathrm{i}}-\left(\frac{\Delta \lambda_{\mathrm{n}}+\Delta \lambda_{\mathrm{w}}}{2}\right) \\
& \lambda_{\mathrm{b}}=\lambda_{\mathrm{i}}+\left(\frac{\Delta \lambda_{\mathrm{n}}+\Delta \lambda_{\mathrm{w}}}{2}\right)
\end{aligned}
$$

\section{FIGURE 13. TRAPEZOIDAL SPECTRAL SLIT WIDTH - UNEQUAL SLITS}

The determination of directional response for the spectroradiometer illustrated in Figure 11 is somewhat more complicated than for the basic radiometer discussed in Section 3.4.1. The entrance slit of the monochromator in this instrument also serves as the field stop of the collecting optics. In the usual orientation, with the slits vertical, the entrance slit width establishes the extent of the field in azimuth and the entrance slit height determines its extent in elevation. Mapping the field of view with a distant small source as outlined in Section 3.4.2 will reveal wavelength as well as spatial variations.

Let us consider the situation where the image of a distant small source is much smaller than the width of the entrance slit and falls entirely within the space between the slit jaws. In this situation the source image acts as a narrower entrance slit (as in the so-called slitless spectrographs used for star spectra). 
The fact that it is narrower will not change the overall spectral slit width of the instrument, as long as the exit slit is at least as wide as the entrance slit to begin with (see Figure 13). However, any change in the horizontal position of the small image across the width of the entrance slit results in a change in the wavelength of the ray from the center of this image, or reduced entrance slit, to the center of the exit slit (i.e. a change in the instantaneous wavelength setting $\lambda_{i}$ ). The resulting uncertainty thus introduced in the wavelength scale is obviously less than the spectral slit width of the entrance slit. If this degree of uncertainty cannot be tolerated, the correct wavelength setting can be computed from a boresight camera record or other determination of the exact position of the distant small source in the field [15]. Similar wavelength scale changes together with some degradation of spectral resolution can be expected when the source is moved in elevation off the optic axis. The analysis of these changes is complicated and differs considerably for different dispersing elements, such as gratings and prisms. Actual exploration of the field as outlined in Section 3.4.2 is the only sure way to determine the variation of responsivity with direction, and the wavelength shift with azimuth and elevation changes must be taken into account in order to interpret correctly the results of such a mapping.

In the treatment of the temporal variation of responsivity (Section 3.5) a caution was presented in regard to spatially scanning radiometers. This use of radiometer can limit the spatial resolution of the instrument if the bounds of the radiometer frequency response are exceeded. In spectroradiometers the same caution applies in regard to spectral scanning. Just as the effective field of view of a scanning radiometer can be determined by the relationship between the spatial scan speed and the frequency response, so the spectral resolution of a spectroradiometer can be affected by the speed of its wavelength scanning acting with its frequency response.

The problem of determining the spectral distribution of the radiation incident at the entrance slit, $\mathrm{P}_{\lambda}(\lambda)$, from the instrument output, $\mathrm{V}\left(\lambda_{i}, \Delta \lambda_{s}\right)$, and the instrument parameters, $R_{D}\left(\lambda_{m}\right), s(\lambda)$ and $\tau_{s}(\lambda)$, by solving the integral Equation 36 is a very difficult one. While various methods for dealing with such a convolution integral can be suggested on purely mathematical grounds, it appears that they are impractical for data reduction computations in most instances because of their sensitivity to the presence of noise (which can be regarded as another perturbation or distortion of $\tau_{s}(\lambda)$ in the output $\left.V\left(\lambda_{i}, \Delta \lambda_{s}\right)\right)$. The approximation method that is most generally used, while not entirely satisfactory, enjoys the tremendous advantage of simplicity.

By successive applications of the mean value theorem of integral calculus, the integral Equation 36 above can be transformed to

$$
\mathrm{V}\left(\lambda_{\mathrm{i}}, \Delta \lambda_{\mathrm{s}}\right)=\mathrm{R}_{\mathrm{D}}\left(\lambda_{\mathrm{m}}\right) \mathrm{P}_{\lambda}\left(\lambda_{1}\right) \mathrm{s}\left(\lambda_{2}\right) \int_{\lambda_{\mathrm{a}}}^{\lambda_{\mathrm{b}}^{\mathrm{b}}} \tau_{\mathrm{s}}(\lambda) \mathrm{d} \lambda=\mathrm{R}_{\mathrm{D}}\left(\lambda_{\mathrm{m}}\right) \mathrm{P}_{\lambda}\left(\lambda_{1}\right) \mathrm{s}\left(\lambda_{2}\right) \Delta \lambda_{\mathrm{s}}
$$

where

$$
\lambda_{1} \text { and } \lambda_{2} \text { are wavelengths within the interval }\left(\lambda_{a}, \lambda_{b}\right) \text { at which } P_{\lambda}(\lambda) \text { and } s(\lambda) \text { have their }
$$


mean values, respectively.

In the usual treatment, one replaces these mean values with the values at the central wavelength, $\lambda_{i}$. The exactness of this procedure depends upon the amount of variation in $R_{D}(\lambda), s(\lambda)$ and $P_{\lambda}(\lambda)$ within the inter$\operatorname{val}\left(\lambda_{\mathrm{a}}, \lambda_{\mathrm{b}}\right)$. If these variations lead to too great errors, then the problem of solving Equation 36 requires a reexamination. The use of a smaller value of $\Delta \lambda_{s}$ (i.e., higher resolution) or a different calibration source with a $\mathrm{P}_{\lambda}(\lambda)$ which is more uniform in the interval, may allow the use of the method outlined above. Note that, in terms of the instrument spectral power responsivity, Equation 38 can be rewritten as:

$$
\mathrm{V}\left(\lambda_{i}, \Delta \lambda_{s}\right)=R_{P_{\lambda}}\left(\lambda_{i}\right) P_{\lambda}\left(\lambda_{i}\right) \Delta \lambda_{s}
$$

\subsection{ABSOLUTE VS. RELATIVE CALIBRATION OF SPECTRORADIOMETERS}

It is clear from the foregoing that any measurements (including calibration measurements) with spectroradiometers are extremely difficult, particularly in the field where all of the pertinent factors are not easily controlled or accounted for. For this reason investigators sometimes find it easier to use a spectral instrument for the measurement of only the relative spectral distribution $r(\lambda)$, rather than an absolute measurement of $\mathrm{P}_{\lambda}(\lambda)$. This establishes the shape of the spectral curve. The scale factor to establish the correct values of ordinates in absolute units is then obtained by one or more separate measurements with a simpler radiometer, which is more easily calibrated in absolute units. If this is done correctly, it is valid. However, it may be very difficult in many cases to insure that the two instruments, the spectroradiometer from which the shape of the spectral curve is established and the radiometer used to obtain integrated values over a wider band in absolute units, are actually measuring the same beam of radiation. Because of the frequent use of the monochromator entrance slit as the field stop in a scanning spectroradiometer, the shape and size of its field (particularly with the curved slit of some prism instruments) may not correspond at all to that of the radiometer, and the portions of the source and background which lie within the two fields may be quite different. Also, the overall spectral responsivity of the radiometer must be known accurately in order to relate its integrated response correctly to the measured curve of the relative spectral distribution (see Section 3.3.3).

\subsection{INTERFEROMETERS}

High resolution spectroscopy has been carried out in the past by extensive use of interferometers [16 through 21]. These instruments either divide the amplitudes associated with each incident wave front or divide the wave fronts themselves. They include the Fabry-Perot interferometer, the Lummer-Geherke plate, the Michelson Echelon, and the Michelson interferometer. With each instrument except the last multiple beams are used to obtain very sharp interference fringes and thus high resolution. The functions, $\tau_{S}(\lambda)$, 
for these multiple beam instruments are tabulated below.

Fabry-Perot interferometer:

$$
\tau_{\mathrm{S}}(\lambda) \sim\left[\frac{\eta^{2} / 4 \rho}{1+\eta^{2} \sin ^{2} \pi \mathrm{p}}\right]
$$

where

$$
\eta^{2}=\frac{4 \rho}{(1-\rho)^{2}}
$$

$\rho$ is the reflectance of the coatings

$\mathrm{p}$ is the order number

and

$$
\Delta \lambda_{\mathrm{S}}=\frac{\lambda^{2}}{2 \pi \mathrm{t}} \cdot \frac{1-\rho}{\rho}
$$

where

$$
\mathrm{t}=\text { spacer thickness }
$$

Lummer-Geherke plate:

$$
\tau_{S}(\lambda) \sim \frac{(1-\rho)^{2}+4 \rho{ }^{N} \sin ^{2}(\pi \delta N / \lambda)}{(1-\rho)^{2}+4 \rho \sin ^{2}(\pi \delta / \lambda)}
$$

where $\quad \mathrm{N}$ is the number of emergent beams obtained for a given plate length and angle of emergence

$\rho=$ reflectance

$\delta=$ path difference between two successive beams

Michelson Echelon:

$$
\tau_{\mathrm{S}}(\lambda) \sim \mathrm{F}_{1} \cdot \mathrm{F}_{2} \cdot \mathrm{F}_{3}
$$

where $\mathrm{F}_{1}, \mathrm{~F}_{2}$ are factors for the Fraunhofer patterns obtained with rectangular objects (they are of the $\frac{\sin ^{2} \beta}{\beta^{2}}$ form); $F_{3}$ describes the interference pattern due to the $N$ steps of the echelon

In the case of the Michelson interferometer, recent interest has stemmed from the utilization of the fact that the spectral distribution in the fringe patterns is equivalent to the Fourier transform of the spectrum. Signal processing techniques, many of which have been developed in connection with radar work during and since World War II, exist for rapid reconstruction of the spectra from interferograms. These have been reported in the literature [19] and some commercial instruments using these techniques are now available [22].

The amplitude, a, of an incident wave in a Michelson interferometer can be considered to be split equally into two parts by the beam splitter, B (neglecting factors such as phase shift on reflection, the need for compensation plates and other departures from the ideal case) as shown in Figure 14. Here the divided ampli- 
tudes $\mathrm{a} / 2$ are associated with the two paths, $\mathrm{BM}_{1}$ and $\mathrm{BM}_{2}$; and $\mathrm{a}^{\prime}$, the amplitude of the emergent wave, is the result of the vector addition of one half of each of these amplitudes. We assume $M_{1}$ to be perpendicular to $M_{2}$. The emergent intensity distribution is obtained by $a^{\prime *} a^{\prime}$ where $a^{\prime *}$ is the complex conjugate of $a^{\prime}$. Thus the transmittance for a Michelson interferometer for wave fronts which are parallel to $M_{1}$ and $M_{2}$ is:

$$
\tau_{\mathrm{S}}(\lambda) \sim \mathrm{a}^{2} \cos ^{2} \delta / 2
$$

where

$$
\begin{aligned}
& \delta=\frac{4 \pi \mathrm{t}}{\lambda} \\
& t=\text { relative displacement of } M_{1} \text { from the zero phase position, i.e., from } B M_{1}=B M_{2}
\end{aligned}
$$

In the absence of multiple reflections the interference fringes obtained are quite broad as compared to those from a Fabry-Perot interferometer. However, high resolution can still be obtained with long path differences if the problem of overlapping orders can be easily solved. If the movable mirror is oscillated in a sawtooth wave so that the mirror moves at a constant velocity for an appreciable portion of a wave cycle, then the transmission of the interferometer also varies for each wavelength. The output frequencies of such an interferometer-spectrometer are directly related to the wave numbers (the inverse of the wavelength) present in the incoming radiation. Wavelength identification is not difficult in this method of operation. On the other hand, Fabry-Perot interferometers used with large spacers require careful order sorting. There exist graphical means which can greatly simplify this problem [23].

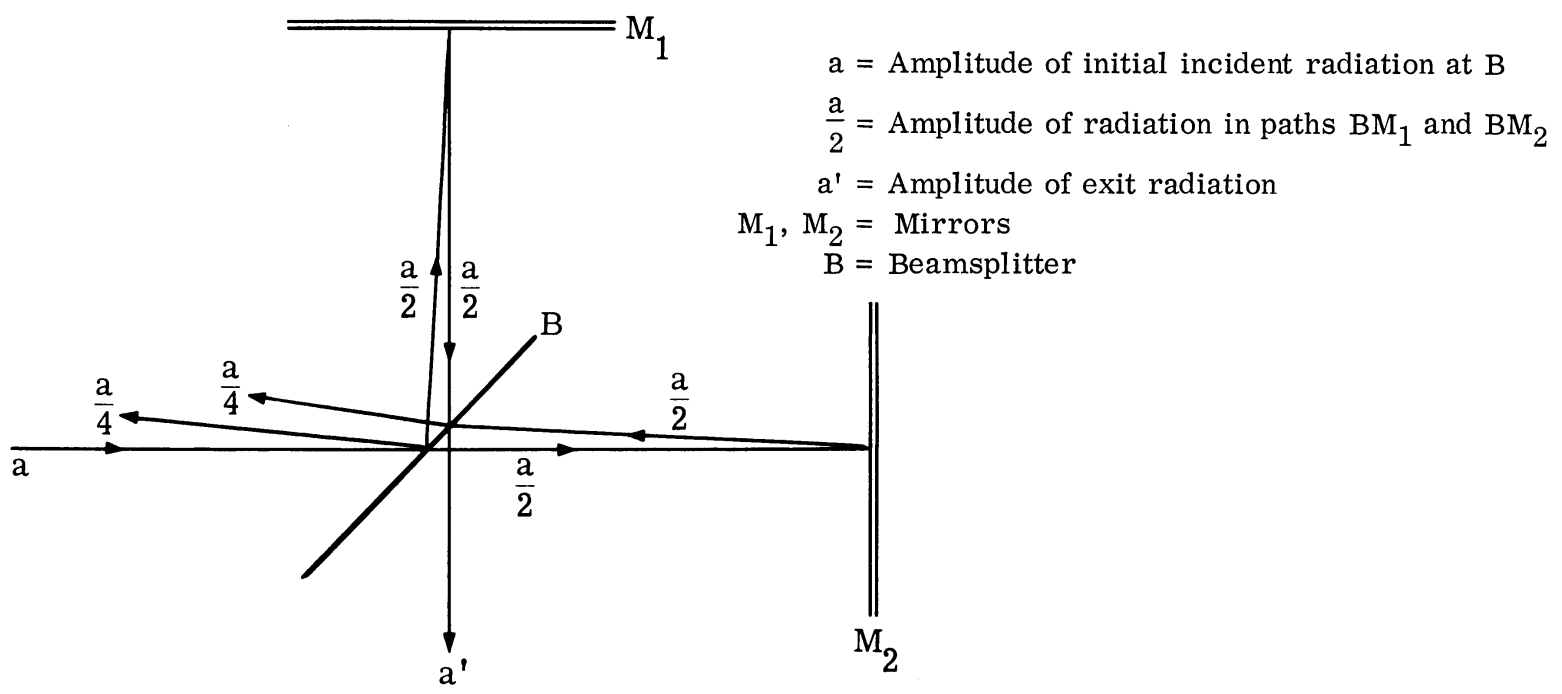

FIGURE 14. MICHELSON INTERFEROMETER

Although interferometers can be regarded as spectrometers, their use as spectroradiometers is not particularly direct. A generally accepted practice is the use of a boresighted radiometer limited to a fixed 
narrow spectral region within the scanned spectral range of the interferometer spectrometer to give a single absolute radiometric value (see Section 5.2). This value (or several such values) can be used to make absolute the relative spectra obtained. Calibration of such instruments, therefore, does not call for procedures basically different from those outlined before. The precautions stated before often take on greater importance for these instruments. Temporal changes of the source radiation at frequencies which can confuse the analysis of the interferometer outputs must be avoided if Fourier analysis methods are employed.

6

\section{CALIBRATION SOURCES}

Any radiative sources whose radiation characteristics are known can be used for calibration. Thus, a heated conical cavity, a heated molecular gas, a thin film of polystyrene, a Geissler tube containing helium at a low pressure, an iron arc - all of these may be used for calibration sources. Radiation, either emitted or absorbed, with known spectral, spatial and temporal distributions, can be classifiable into continua, band and line spectra, or mixtures of these. Discussions of calibration sources are to be found in References 1 , $8,12,13,14,20$ and 24 through 32 . In view of the extensive literature on this subject, it will not be treated here.

SUMMARY

The definitive work on radiometric calibration has yet to be written. It is the opinion of the authors that no paper can treat this subject completely since each instrument requires a separate analysis. In the preceding sections, we have tried to discuss in a general way several topics which have not been covered before in the manner of our approach. We have stressed those items which have been slighted or overlooked in the literature, as well as those which we consider so important as to warrant a reiteration. Still, this report, or any other document, can never be a substitute for a thorough study of the literature in this field. We can learn through a review of the procedures used by our many fellow workers in radiometric research. For this reason, we have added a bibliography of articles we have found to be useful. A search through them will lead one to many more papers relating to radiometric calibration.

The most useful summary of this paper may be prepared best by the reader. It should be in the form of a summary check list for the calibration of his own instrument. Its preparation involves carefully reviewing this report and examining such questions as: 
Do the calibration conditions reproduce, as closly as possible, all of the pertinent conditions of the measurements which will be based on this calibration? Are the extremes in level of radiation covered? Have spatial, spectral and temporal dependencies been reproduced during the calibration? To the extent that they have not, are the differences adequately recognized and treated in the measurement, data-reduction, and reporting procedures?

Have all of the possible variations in the instrument functions and methods of operation been calibrated in terms, not only of their individual effects, but also of the interactions between functions and operations?

A final remark should be made. Not long ago laboratory instruments were at least assembled, if not made, by the scientist destined to use them in research. Today commercial instruments, completely packaged and partially or wholly automated, are purchased for the largest number of laboratory and field studies. Unfortunately, too many investigators have accepted these instruments as black boxes whose interiors are known only through the manufacturers' instruction manuals. While we have no longing to return to "the good . old days" when optics were made, coated, mounted and aligned by the scientist himself, still we deplore the other extreme. It does not seem possible to make accurate and precise measurements of the radiation from sources with radiometric instruments whose parts and functions remain unseen and untouched. 


\section{REFERENCES}

1. H. J. Kostkowski and R. D. Lee, Theory and Methods of Optical Pyrometry, National Bureau of Standards Monograph 41, Mar. 1962 (Obtainable from Superintendent of Documents, US Government Printing Office, Washington 25, D. C., for 25\%).

2. Charles Ravitsky, Cameron Cumming, Trevor S. Moss, Standard Procedures for Target and Background Infrared Measurements, Office of the Director of Defense Research and Engineering, Apr. 1962, (obtainable from the Office of Technical Services, Department of Commerce,

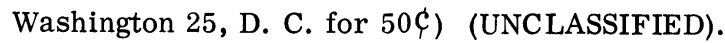

3. "Aims, Conclusions, and Recommendations on Background Information," Part I, Report of The Working Group on Infrared Backgrounds (WGIRB), Report Number 2389-7-S, published by IRIA, Willow Run Laboratories, The University of Michigan, Ann Arbor, July 1957 (CONFIDENTIAL, M-H-A-).

4. "Concepts and Units for the Presentation of Infrared Background Information," Part III, Report of the Working Group on Infrared Backgrounds (WGIRB), Report Number 2389-3-S, published by IRLA, Willow Run Laboratories, The University of Michigan, Ann Arbor, Nov。 1956 (UNCLASSIFIED).

5. "Proceedings of the Symposium on Infrared Backgrounds," Report Number 2389-2-S, IRIA, Willow Run Laboratories, The University of Michigan, Ann Arbor, Oct. 1956 (CONFIDENTIAL).

6. N. Ginsburg, W. R. Frederickson and R. Paulson, "Measurements with a Spectral Radiometer," J. Opt. Soc. Am., Dec. 1960, Vol. 50, p. 1176.

7. R. A. Oetjen, E. E. Bell, J. Young, and L. Eisner, "Spectral Radiance of Sky and Terrain at Wavelengths Between 1 and 20 Microns," J. Opt. Soc. Am., Dec. 1960, Vol. 50, p. 1308.

8. M. R. Holter, et al, Fundamentals of Infrared Technology, Macmillan, New York, 1962.

9. "Infrared Target and Background Radiometric Measurements: Concepts, Units and Techniques," Report of the Working Group on Infrared Backgrounds (WGIRB), Report Number 2389-64-T, published by IRIA, Institute of Science and Technology, The University of Michigan, Ann Arbor, Jan. 1962 (UNCLASSIFIED).

10. E. Bell, "Radiometric Quantities, Symbols and Units," Proc. IRE, 1959, Vol. 47, p. 1432.

11. J. W. T. Walsh, Photometry, Constable and Company, Ltd., London, and Robert MacLehose and Co., Ltd, Glasgow (2nd ed., rev. 1953).

12. R. A. Sawyer, Experimental Spectroscopy, Prentice-Hall, Inc., New York, 1946.

13. G. R. Harrison, R. Lord, and J. R. Loofbourow, Practical Spectroscopy, Prentice-Hall, New York, 1948.

14. E. K. Plyler, A. Danti, L. R. Blaine, and E. D. Tidwell, Vibration-Rotation Structure in Absorption Bands for the Calibration of Spectrometers from 2 to 16 Microns, National Bureau of Standards Monograph 16,'21 June 1960. (Available from Superintendent of Documents, US Government Printing Office, Washington 25, D。C., for 20c) Reprinted from the Journ. Nat. Bur. Stnds. A. Physics and Chemistry, Jan. - Feb., 1960, Vol. 64A.

15. W. W. Talbert, D. E. Matlack, H. A. Templin, R. E. Morrison, and T. J. Brahmey, "Spectral Measurements of Ballistic Missile Plumes (1.0-5.5 Microns) (U)," NAVORD Report 6755, U. S. Naval Ordnance Laboratory, White Oak, Md., 15 Dec. 1959, pp. 3-4 (SECRET).

16. K. W. Meissner, "Interference Spectroscopy, Part I and Part II," J. Opt. Soc. Am., June 1941, Vol. 31, pp. 405-427, and April 1942, Vol. 32, pp. 185-211. 
17. C. Candler, Modern Interferometry, Hilger and Watts Ltd., London, Hilger Division, 1951.

18. W. E. Williams, Applications of Interferometry, J. Wiley and Sons, New York, 1950.

19. Interferometry, NPL Symposium No. 11, 9-11 June 1959, National Physical Laboratory via London: Her Majesty's Stationery Office, 1960.

20. S. Tolansky, High Resolution Spectroscopy, Pitman Publishing Corp., New York, 1947.

21. R. W. Ditchburn, Light, Interscience Publishers, Inc., New York, 1957 (rev.).

22. Private Communication, Block Associates, Cambridge, Mass.

23. G. V. Deverall, K. W. Meissner, and G. J. Zissis, "Section of Optimal Spacers in PerotFabry Interferometry," J. Opt. Soc. Am., 1953, Vol. 43, pp. 673-680.

24. W. E. Forsythe, Measurement of Radiant Energy, McGraw-Hill, New York, 1937.

25. J. Strong, Procedures in Experimental Physics, Prentice-Hall, New York, 1938.

26. A. Gouffee, "Corrections d'ouverture des corps noirs artificiels compe tenu des diffusions multiples internes," Rev. Opt., 1945, Vol. 24.

27. J. C. DeVos, "Evaluation of the Quality of a Blackbody," Physica,1954, Vol. 20, p. 669.

28. A. LaRocca and G. J. Zissis, "Field Sources of Blackbody Radiation," Rev. Sci. Instr., 1959, Vol. 30, p. 200.

29. M. M. Benarie, "Optical Pyrometry below Red Heat,", J. Opt. Soc. Am., 1947, Vol. 47, p. 1005.

30. W. L. Eisenman and A. J. Cussen, "A Comparative Study of Several Black Bodies," Proc. IRIS, 1956, Vol. 1, No. 1, p. 39.

31. G. T. Lolas, R. J. Coruccini, and H. P. Broida, "Design and Construction of a Blackbody and Its Use in the Calibration of a Grating Spectroradiometer," Rev. Sci. Instr., 1958, Vol. 29, p. 505.

32. C. S. Williams, "Discussion of the Theories of Cavity-Type Sources of Radiant Energy," J. Opt. Soc. Am., 1961, Vol. 51, p. 564. 


\section{BIBLIOGRAPHY}

Calculation Aids and/or Equations

Benford, Frank, "Projection of Light," J. Opt. Soc. Am., 1945, Vol. 35, p. 149.

Benford, Frank, "True-Area Photometric Distribution Curve," J. Opt. Soc. Am., 1942, Vol. 32, p. 307.

Benford, Frank, "Radiation in a Diffusing Medium," J. Opt. Soc. Am., 1946, Vol. 36, p. 524.

Benford, Frank, and Bock, John E., "Chart for Transferring Spherical Coordinates,"J. Opt. Soc. Am., 1941, Vol. 31, p. 239.

Bracewell, Ronald N., "Simple Graphical Method of Correcting for Instrumental Broadening," J. Opt. Soc. Am., Oct. 1955, Vol. 45, No. 10, p. 873.

Eppley, Marion, and Karoli, Alton R., "Use of Wave Number in Radiation Formulas," J. Opt. Soc. Am., Nov. 1953, Vol. 43, p. 957.

Melvin, M. A., "Blackbody Radiation and Lambert's Law," Am. J. Phys., 1955, Vol. 23, p. 508.

Pinovonsky and Nagel, "Tables of Blackbody Radiation Functions," Macmillan, New York, N. Y., 1961 (Book Review, Applied Optics, May 1962, Vol. 1, No. 3, p. 334).

Calibration

Bethke, George W., "Convenient Calibration Curve for Prism Infrared Spectrometers," J. Opt. Soc. Am., July 1956, Vol. 46, No. 7, p. 560.

Ching-Sung Yu, Absolute Calibration of a Low Brightness Source, Report Number AR-16 (AD 55 769), Harvard University, Cambridge, Mass. (UNCLASSIFIED).

Christensen, R. L., and Ames, I., "Absolute Calibration of a Light Detector," J. Opt. Soc. Am., Feb. 1961, Vol. 51, No. 2, p. 224.

Coates, Vincent J., and Hausdorff, Harry, "Interferometric Method of Measuring the Spectral Slit Width of Spectrometers," J. Opt. Soc. Am., June 1955, Vol. 45, No. 6, p. 425.

Ebers, E. S., and Nielsen, H. H., "A Method for Increasing the Life of Nernst Glowers," Rev. Sci. Instr.,Dec. 1940, No. 11, p. 429.

Filler, A. S. ,and Indyk, L., "Calibration of Infrared Prism Spectrometers," J. Opt. Soc. Am., May 1961, Vol. 51, No. 5, p. 572.

Fisher, Russell A., "Interference Method for Calibration of Recording Spectrographs," J. Opt. Soc. Am., Nov. 1959, Vol. 49, No. 11, p. 1100.

Glatt, Leonard, "Procedure for Evaluating the Small Object Detecting Capability of an Electron-Scanned Image Tube," J. Opt. Soc. Am., Dec. 1959, Vol. 49, No. 12, p. 1209.

Henry, W. G., and Heharry, M. R., "Calibration of a Prism Spectrometer," J. Opt. Soc. Am., Mar. 1961, Vol. 51, No. 3, p. 356.

Ingel, J. W., Barnes Model R-802, Radiometer Sources of Discrepancy Between Different Methods of Calibration, Working Paper Number W-58-820-1, U. S. Naval Avionics Facility, Oct. 1958 (UNCLASSIFIED).

Kaspar, Josef, Spectral Intensity Calibration of Infrared Spectrometers, WADC Tech. Report Number 52-58, Aircraft Radiation Lab., WADC, Wright-Patterson Air Force Base, Dayton, O., April 1952 (UNCLASSIFIED). 
Kay, K. K., and Barrett, H. M., "A New Method for the Determination of the Transmission Factor of a Spectroradiometer," J. Opt. Soc. Am., 1937, Vol. 27, p. 65.

Kostkowski, Henry J., and Bass, Arnold M., "Slit Function Effects in the Direct Measurement of Absorption Line Half-Widths and Intensities," J. Opt. Soc. Am., Dec. 1956, Vol. 46, No. 12 , p. 1060.

Lempicki, A., Samelson, H., and Brown, A., "Slit-Width Error in the Measurement of Absorption Constants," J. Opt. Soc. Am., Jan. 1961, Vol. 51, No. 1, p. 35.

Levialdi, A., (trans. by Darrow, K. K.), "On A Scheme for Mounting Globar for Researchers in the Infrared," Rev. Sci. Instr.,Dec. 1940, No. 11, p. 429.

Moran, Hubert S., "Determination of the Relative Spectral Sensitivity of Phototubes," J. Opt. Soc. Am., Jan. 1955, Vol. 45, No. 1, p. 12.

Normand, Sir Charles W. B., and Kay, R. H., "Notes on the Design Adjustment and Calibration of Spectrophotometers," J. of Sci. Instr.,Feb. 1952, Vol. 29, No. 2, p. 33.

Oetjen, Robert A., Bell, Ely E., Young, James, and Eisner, Leonard, "Spectral Radiance of Sky and Terrain at Wavelengths between 1 and 20 Microns. I. Instrumentation," J. Opt. Soc. Am., Dec. 1960, Vol. 50, No. 12, p. 1308.

Rao, K. Narahari, Coburn, T. J., Garling, J. S., Rossmann, K., and Nielsen, H. H., "Wavelength Calibrations in Infrared. Part II. Use of Atomic Lines from a Hollow Cathode Discharge Tube with Neon as Carrier Gas," J. Opt. Soc. Am., Mar. 1959, Vol. 49, No. 3, p. 221.

Rao, K. Narahari, Ryan, L. R., and Nielsen, Harold H., "Wavelength Calibrations in Infrared. Part I. Some Problems Concerning the Determination of Absolute Positions of Infrared Lines," J. Opt. Soc. Am., Mar. 1959, Vol. 49, No. 3, p. 216.

Schreiber, P. W., Fabry-Perot Interferometer Used for Wavelength Calibrations in the Infrared, Report Number 7, Ohio State University, Columbus, O., April 1957 (UNCLASSI$\overline{\text { FIED). }}$

Shepherd, G. G., Absolute Calibration of a Low Brightness Source, Report Number AR-16 (AD 55 769), University of Saskatchewan, Saskatoon, Canada (UNCLASSIFIED).

VonPlanta, Peter C., "Experimental Study of the Slit Function of an Ebert Spectrometer in the Visible and Near-Infrared," J. Opt. Soc. Am., July 1957, Vol. 47, No. 7, p. 629.

Wyszecki, Gunter, "Multifilter Method for Determining Relative Spectral Sensitivity Functions of Photoelectric Detectors," J. Opt. Soc. Am., Oct. 1960, Vol. 50, No. 10, p. 992.

Radiometry/Spectrometry

Astheimer, Robert W., and Wormser, Eric M., "High-Speed Infrared Radiometers," J. Opt. Soc. Am., Feb. 1959, Vol. 49, No. 2, p. 179.

Astheimer, Robert W., and Wormser, Eric M., "Instruments for Thermal Photography," J. Opt. Soc. Am., Feb. 1959, Vol. 49, No. 2, p. 184.

Babrov, H. J.,"Instrumental Effects in Gas Spectra and Spectroscopic Temperature Measurements," J. Opt. Soc. Am., Feb. 1961, Vol. 51, No. 2, p. 171.

Bell, Ely E., Burnside, Phillip B., and Dickey, Frederick P., "Spectral Radiance of Some Flames and Their Temperature Determination," J. Opt. Soc. Am., Dec. 1960, Vol. 50, No. 12 , p. 1286.

Benford, Frank, "Mllumination From Extended Sources Computed by the Theory of the Integrating Sphere," J. Opt. Soc. Am., 1940, Vol. 30, p. 33. 
Benford, Frank, "Temperature Corrections in Optical Pyrometry," J. Opt. Soc. Am., 1939, Vol. 29, p. 162 .

Bethke, George W., "Ultrarapid-Scan Infrared Spectrometer," J. Opt. Soc. Am., Nov. 1960, Vol. 50, No. 11, p. 1054.

Bullock, B. W., and Silverman, S., "A Rapid Scanning Spectrometer for Oscillograph Presentation in the Near Infrared," J. Opt. Soc. Am., Sept. 1950, Vol. 40, No. 9.

Dikar, R. V. Karan, Improvement at Spectroscopic Recording Techniques, Final Report Number AFCRC-TR-57-280, Boston University, Boston, Mass., 1 Mar. 1957 (UNCLASSIFIED).

Dunning, C. E., Infrared Radiometry with Selective Detectors, Tech. Memo Number 520:5912-447, Aerojet-General Corp., Azusa, Calif., 10 Mar. 1959 (UNCLASSIFIED).

Einhorn, H. D., and Cohen, A. E. Z., "Stray Light in a Spectrophotometer Caused by Internal Reflection in a $60^{\circ}$ Prism," J. Opt. Soc. Am., Mar. 1954, Vol. 44, p. 232.

Eppley, Marion, and Karoli, Alton R., "Absolute Radiometry Based on a Change in Electrical Resistance," J. Opt. Soc. Am., Aug. 1957, Vol. 47, No. 8, p. 748.

Gibbons, Mathew G., "Experimental Study of the Effect of Field of View on Transmission Measurements," J. Opt. Soc. Am., July 1959, Vol. 49, No. 7, p. 702.

Gibbons, Mathew G., "Radiation Received by an Uncollimated Receiver from a $4 \pi$ Source," J. Opt. Soc. Am., Aug. 1958, Vol. 48, No. 8, p. 550.

Gilmore, H. F., "Some Effects of Aperture Compensation on the Signal-to-Noise Ratio and Resolution of Spectrometers," J. Opt. Soc. Am., 1957, Vol. 47, p. 1057.

Ginsburg, N., Fredrickson, W. R., and Paulson, R., "Measurements with a Spectral Radiometer," J. Opt. Soc. Am., Dec. 1960, Vol. 50, No. 12, p. 1176.

Golay, Marcel J. E., "Multi-Slit Multipass Spectrometers," J. Opt. Soc. Am., Jan. 1953, Vol. 43, No. 1, p. 58.

Golay, Marcel J. E., "Multi-Slit Spectrometry," J. Opt. Soc. Am., June 1949, Vol. 39, No. 6, p. 437.

Golay, Marcel J. E., "Static Multislit Spectrometry and Its Application to the Panoramic Display of Infrared Spectra," J. Opt. Soc. Am., July 1951, Vol. 41, No. 7, p. 468.

Greenler, Robert G., "Interferometry in the Infrared," J. Opt. Soc. Am., 1955, Vol. 45, p. 788.

Hagness, T. R., Zscheile, F. P., Jr., and Sidwall, A. E., Jr., "Photoelectric Spectrometry," J. Phys. Chem., 1937, Vol. 41, p. 379.

Harrison, Thomas R., Radiation Pyrometry and Its Underlying Principles of Radiant Heat Transfer, John Wiley and Sons, New York, N. Y., 1960 (Review in Physics Today, Dec. 1960, p. 56).

Jacquinot, P., "New Developments in Interference Spectroscopy," Reports on Progress in Physics XXIII, 1960.

King, Gilbert W., and Emslie, A. G., "Spectroscopy From the Point of View of Communication Theory, Part I. Resolution," J. Opt. Soc. Am., June 1951, Vol. 41, p. 405.

King, Gilbert W., and Emslie, A. G., "Spectroscopy From the Point of View of Communication Theory, Part II, Line Widths," J. Opt. Soc. Am., Aug. 1953, Vol. 43, p. 658.

King, Gilbert W., and Emslie, A. G., "Spectroscopy From the Point of View of Communication Theory, Part III. Amount of Information in a Spectrum," J. Opt. Soc. Am., Aug. 1953, Vol. 43, p. 664. 
King, Gilbert W., and Emslie, A. G., "Spectroscopy From the Point of View of Communication Theory, Part IV. Automatic Recording of Infrared Spectra on Punched Cards," J. Opt. Soc. Am., May 1954, Vol. 44, p. 397.

McPherson, Paul M., Sclar, Nathan, Linden, Bernard R., Brouwer, Willem, and Stair, A. T., Jr., "Photodetecting Instrument with Flat Wavelength Response," J. Opt. Soc. Am., July 1961, Vol. 51, No. 7, p. 767.

Oetjen, Robert A., Bell, Ely E., Young, James, and Eisner, Leonard, "Spectral Radiance of Sky and Terrain at Wavelengths between 1 and 20 Microns. I. Instrumentation," J. Opt. Soc. Am., Dec. 1960, Vol. 50, No. 12, p. 1308.

Porter, John F., "Rapid Scan Infrared Spectrograph with Linear Wavelength Presentation," J. Opt. Soc. Am., July 1961, Vol. 51, No. 7, p. 789.

Pritchard, Benjamin S., "Measurement of Stray Light in a Monochromator," J. Opt. Soc. Am., May 1955, Vol. 45, p. 356.

Schreiber, P. W., Fabry-Perot Interferometer Used for Wavelength Calibrations in the Infrared, Report Number 7, Ohio State University, Columbus, O., April 1957 (UNCLASSIFIED).

Shurcliff, W. A., "Multi-Slit Double Monochromator Using No Moving Parts," J. Opt. Soc. Am., Dec. 1949, Vol. 39, No. 12, p. 1048.

Stair, Ralph, "Filter Radiometry and Some of Its Applications," J. Opt. Soc. Am., Nov. 1953, Vol. 43 , p. 971.

Wensel, H. T., Judd, D. B., and Roeser, Wm. F., "Establishment of a Scale of Color Temperature," NBS Jour. Res., 1934, No. 12, p. 527 (Research Paper 677).

Wilson, Raymond H. Jr., "Using the Sun to Measure Temperatures of Flames in the Laboratory," Astron. Soc. of the Pac., 1952, No. 64, p. 105.

Barbrow, Louis E., "Memorandum on a Procedure for Obtaining Spectral Radiant Intesities of Tungsten-Filament Lamps, 400-700 mu," J. Opt. Soc. Am., Nov. 1959, Vol. 49, No. 11, p. 1122 .

Barnes, B. T., Forsythe, W. E., "Total Emissivity of Various Materials at $100-500^{\circ} \mathrm{C}$," J. Opt. Soc. Am., 1947, Vol. 37, p. 804.

Blitzer, Leon, Evaluation of Infrared Sources, Final Report, Stanford Research Institute, Menlo Park, Calif. (UNCLASSIFIED).

Bluford, Benjamin L. Jr., Design of Planckian Radiators, Technical Memo. Number M-1580, Signal Corps Engineering Labs., Evans Signal Lab., Ft. Monmouth, N. J., May 1954 (UNCLASSIFIED).

Coblenz, W. W., and Stair, R., "The Present Status of the Standards of Thermal Radiation Maintained by the Bureau of Standards," National Bureau of Standards Res. Paper 578, (J. Res. Nat. Bur. Std., No. 11, p. 79, July 1933).

Condell, William J., and Byrne, Grancis T., "Spectral Intensity of ac Operated Tungsten Lamps," J. Opt. Soc. Am., Dec. 1957, Vol. 47, p. 1135.

Cox, J. T., Hass, G., and Jacobus, G. F., "Infrared Filters of Antireflected Si, Ge, InAs, and InSb," J. Opt. Soc. Am., July 1961, Vol. 51, No. 7, p. 714.

Ebers, E. S., and Nielsen, H. H., "A Method for Increasing the Life of Nernst Glowers," Rev. Sci. Instr., Dec. 1940, No. 11, p. 429.

Edwards, David F., The Emissivity of a Conical Black Body, Report Number 2144-105-T, Willow Run Laboratories, The University of Michigan, Ann Arbor, Michigan, November 1956 (UNCLASSIFIED). 
Fisher, Heinz, (AFCRC), "Simple Submicrosecond Light Source with Extreme Brightness," J. Opt. Soc. Am., 1957, Vol. 47, p. 981.

Forsythe, W. E., and Adams, E. Q., "Radiating Characteristics of Tungsten and Tungsten Lamps," J. Opt. Soc. Am., 1945, Vol. 35, p. 306.

Greay, David S., Mark, Peter, and Haskell, Stanley, "New Class of Wide-Range Logarithmic Circuit for a Light-Intensity Meter," J. Opt. Soc. Am., Jan.1960, Vol. 50, No. 1, p. 40.

Harris, Louis, and Cuff, Kermit F., "Reflectance of Goldblack Deposits, and Some other Materials of Low Reflectance from $254 \mathrm{mu}$ to $1100 \mathrm{mu}$. The Scattering-Unit-Size in Goldblack Deposits," J. Opt. Soc. Am., Mar. 1956, Vol. 46, No. 3, p. 160.

Harris, Louis, "The Transmittance and Reflectance of Gold Black Deposits in the 15- to 100-Micron Region," J. Opt. Soc. Am., Jan. 1961, Vol. 51, No. 1, p. 80.

Hass, George, "Filmed Surfaces for Reflecting Optics," J. Opt. Soc. Am., Nov. 1955, Vol 45, No. 11, p. 945.

Jenness, James R. Jr., "Auxiliary Optical System for Measurement of the Infrared Transmittance of Flat Plates with the Perkin-Elmer 12-C Spectrometer," J. Opt. Soc. Am., Aug. 1955, Vol. 45, No. 8, p. 671.

Karoli, A. R., Angstrom, A. K., Drummond, A. J., "Dependence on Atmospheric Pressure of the Response Characteristics of Thermopile Radiant Energy Detectors," J. Opt. Soc. Am., Aug. 1960, Vol. 50, No. 8, p. 758.

Larrabee, Robert D., "Spectral Emissivity of Tungsten," J. Opt. Soc. Am., June 1959, Vol. 49, No. 6, p. 619.

Levialdi, A. (trans. by K. K. Darrow), "On a Scheme for Mounting Globar for Researchers in the Infrared, "Rev. Sci. Instr., Dec. 1940, No. 11, p. 429.

McCarthy, Donald E., "Response Characteristics of a Radiation Thermocouple at Various Pressures," J. Opt. Soc. Am., July 1961, Vol. 51, No. 7, p. 801.

McFee, Raymond H., "Laboratory Source of $1.08 \mu$ and $2.06 \mu$ Monochromatic Radiation," J. Opt. Soc. Am., Jan. 1952, Vol. 42, p. 67.

McGuistan, Richmond B., "On an Approximation to Sinusoidal Modulation, " J. Opt. Soc. Am., Jan. 1958, Vol. 48, No. 1, p. 63.

McQuistan, Richmond B., "On Radiation Modulation," J. Opt. Soc. Am., Jan. 1959, Vol. 49, No. 1, p. 70 .

Knowles, Middleton,W. E., and Mayo, Eleanor G., "Variation in Horizontal Distribution of Light from Candlepower Standards," J. Opt. Soc. Am., Aug. 1951, Vol. 41, No. 8, p. 513.

Moran, Hubert S., "Modification of the Beckman Model DU Spectrophotometer for the Measurement of Interference Filters," J. Opt. Soc. Am., Jan. 1955, Vol. 45, No. 1, p. 26.

Rank, D. H., Bennett, J. M., and Bennett, H. E., "Measurement of Interferometric Secondary Wavelength Standards in the Near Infrared," J. Opt. Soc. Am., July 1956, Vol. 46, p. 477.

Ruper, C. S., A Compact Water-Cooled Carbon Arc Source for Infrared Spectroscopy, Progress Report, Johns Hopkins University, Baltimore, Md., 1 June 1952 (UNCLASSIFIED).

Rupert, C. S., and Strong, John, "The Carbon Arc as an Infrared Source," J. Opt. Soc. Am., July 1950, Vol. 40, pp. 455-459.

Silverman, Shirleigh, "The Emissivity of Globar," J. Opt. Soc. Am., Nov. 1948, Vol. 38, No. 11, p. 989 . 
Stecyk, Adrian,and Fenkelstein, Isidore, Study of Infrared Sources, Report Number 8600, Servo Corporation, New York, 28 December 1959 (UNCLASSIFIED).

Taylor, I. H., Rupert, C. S., and Strong, John, "An Incandescent Tungsten Source for Infrared Spectroscopy," J. Opt. Soc. Am., Sept. 1951, Vol. 41, No. 9, p. 626.

Thomas, Albert L. Jr., Bird, Alvin M. Jr., Development of a Constant Infrared Source, Report Number APGC TN-59-30, Southern Research Institute, Birmingham, Ala., 29 May 1959 (UNCLASSIFIED).

Uhl, Robert J., Infrared Spectral Output of Xenon Flash Tubes, Report Number 7668 TM 3, Servomechanisms Labs., Massachusetts Institute of Technology, Cambridge, Mass., April 1958 (UNCLASSIFIED).

Vandenbelt, J. M., "Holmium Filter for Checking the Wavelength Scale of Recording Spectrophotometers," J. Opt. Soc. Am., July 1961, Vol. 51, No. 7, p. 802. 


\section{DISTRIBUTION LIST}

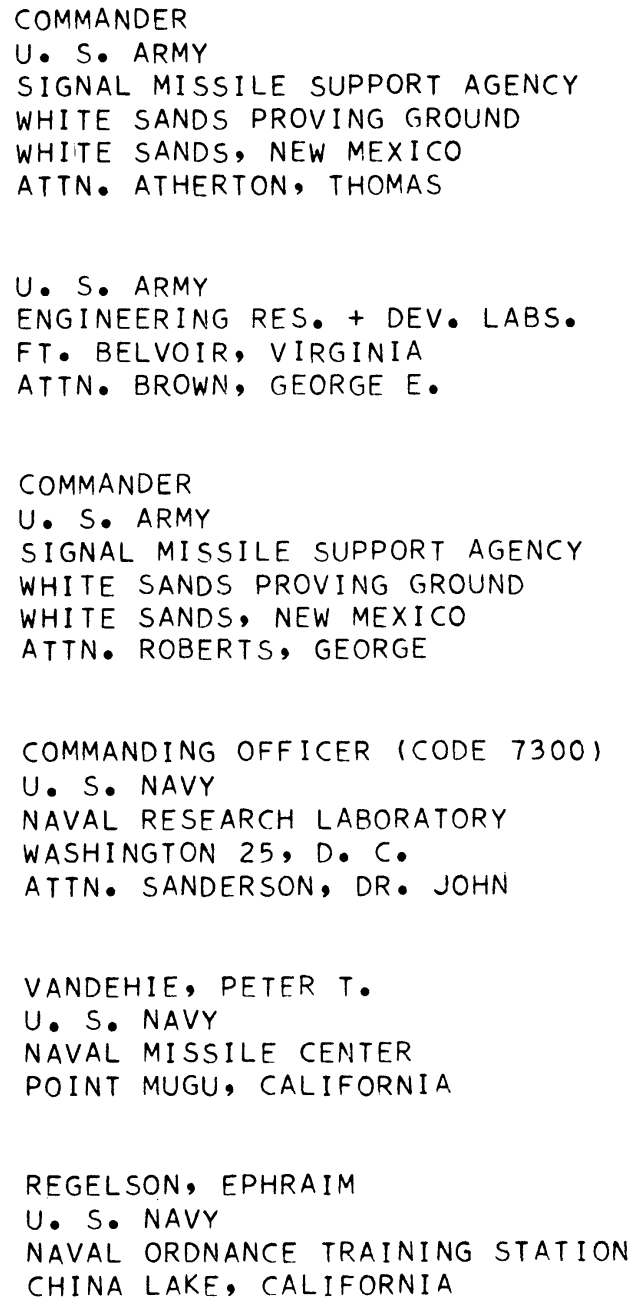

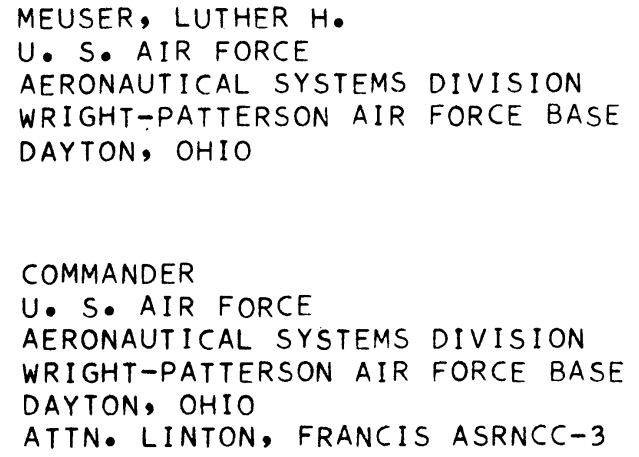




\section{DISTRIBUTION LIST (Continued)}

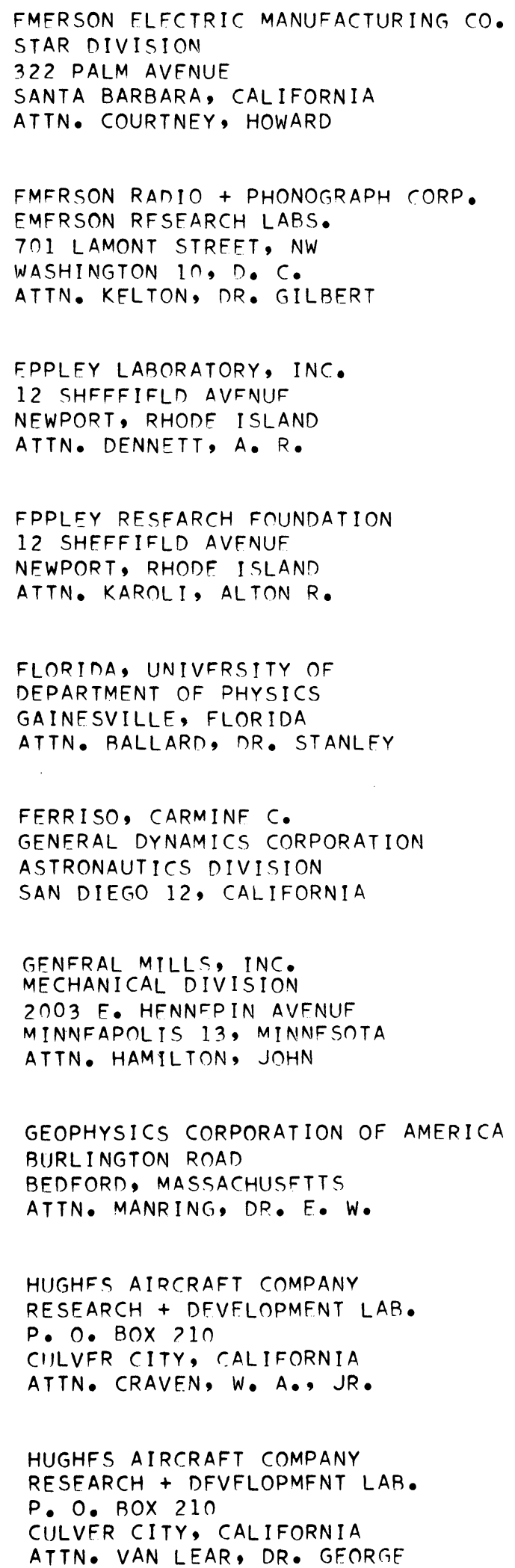




\section{DISTRIBUTION LIST (Continued)}

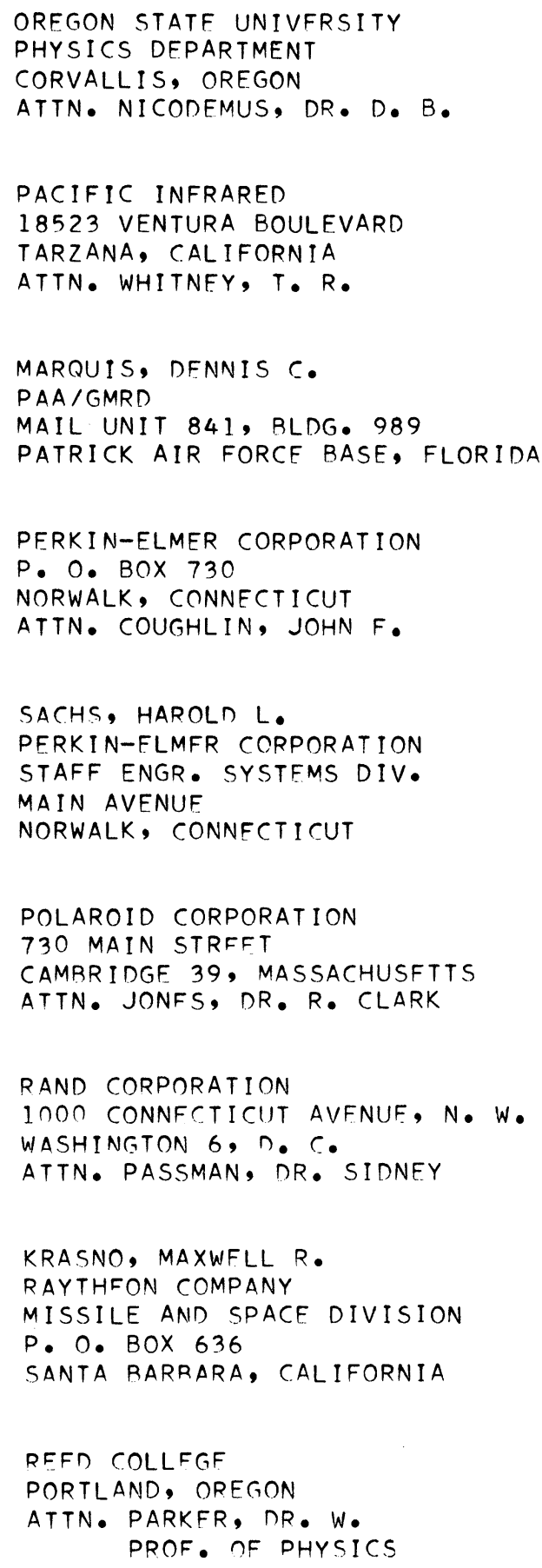




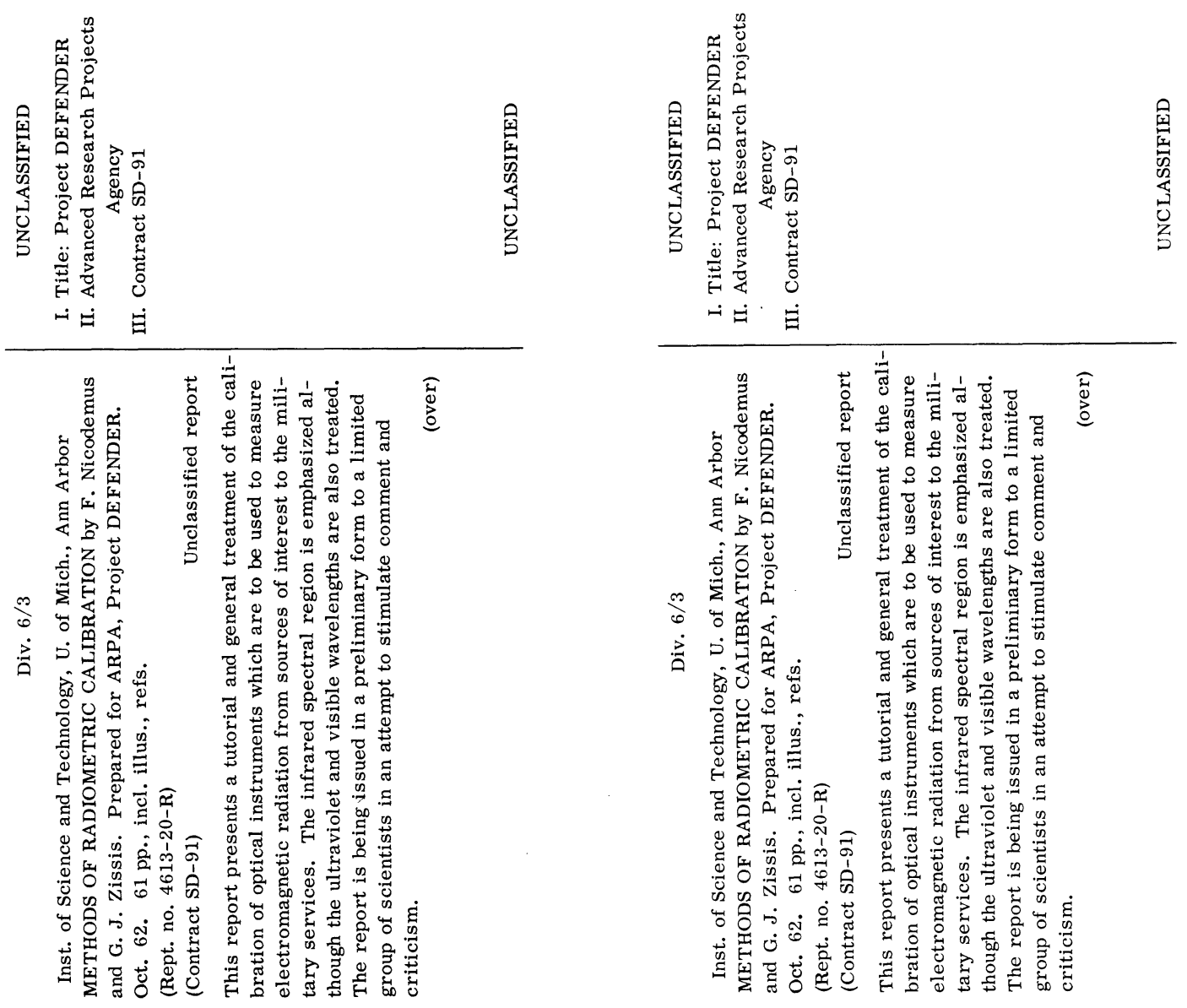

$+$
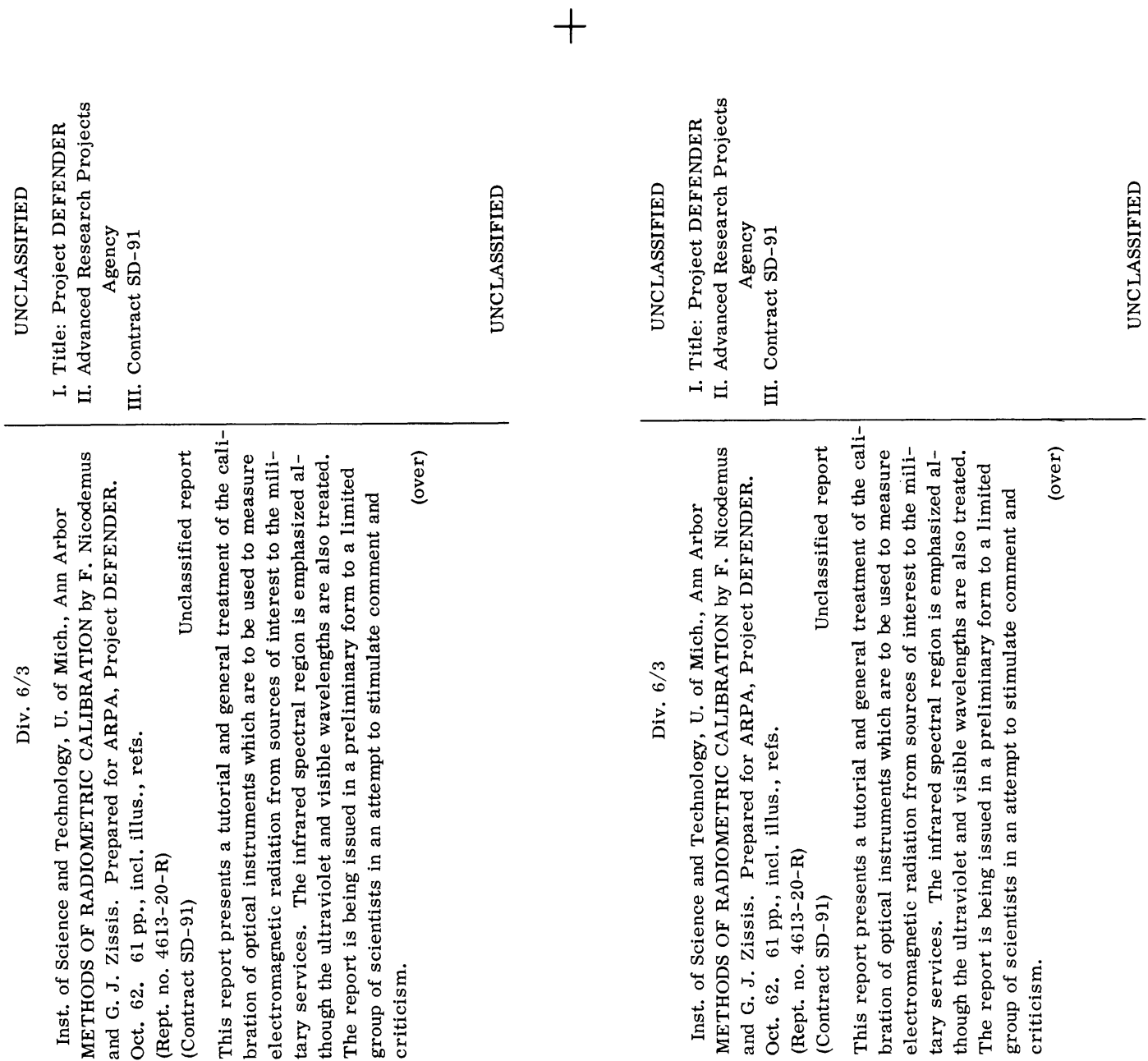

$\rightarrow$

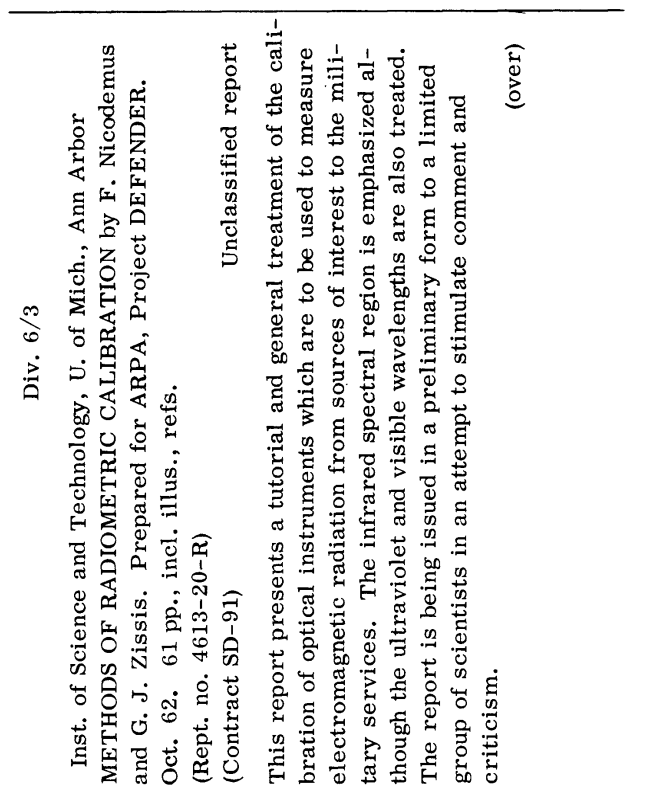



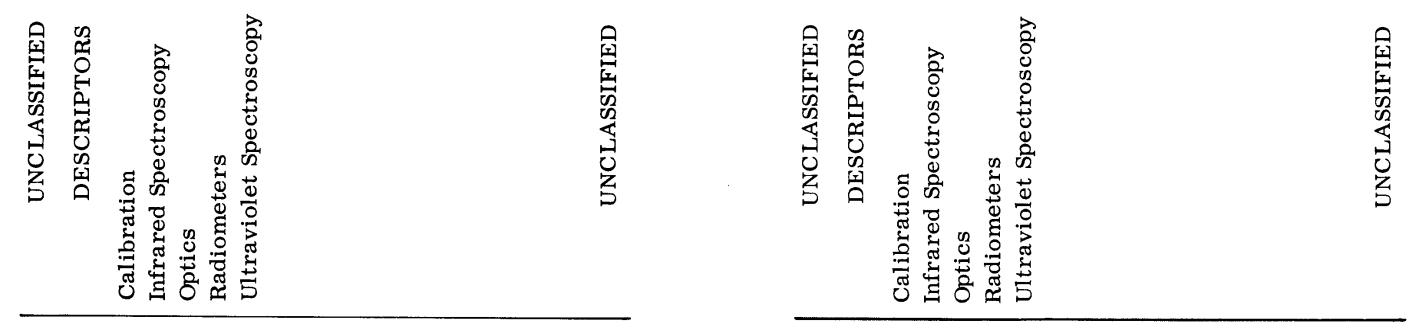

$+$
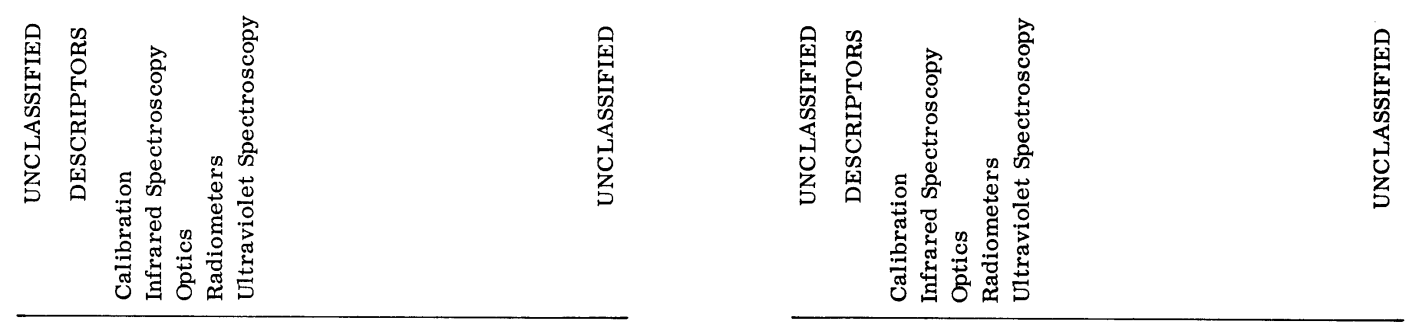

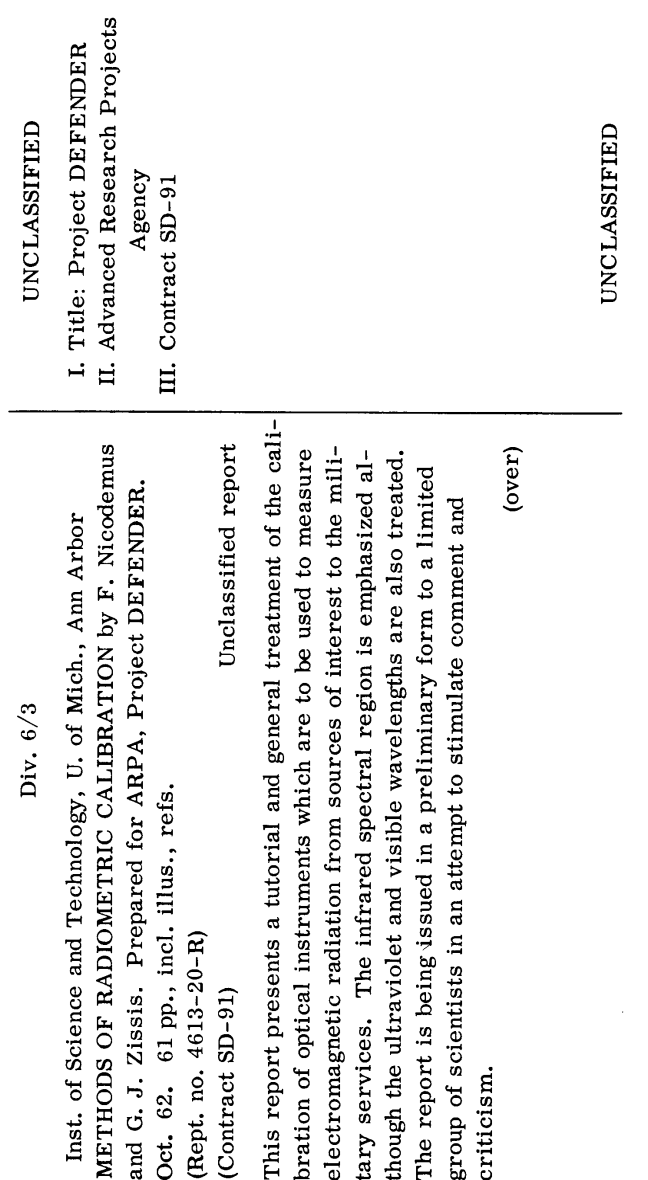

$+$

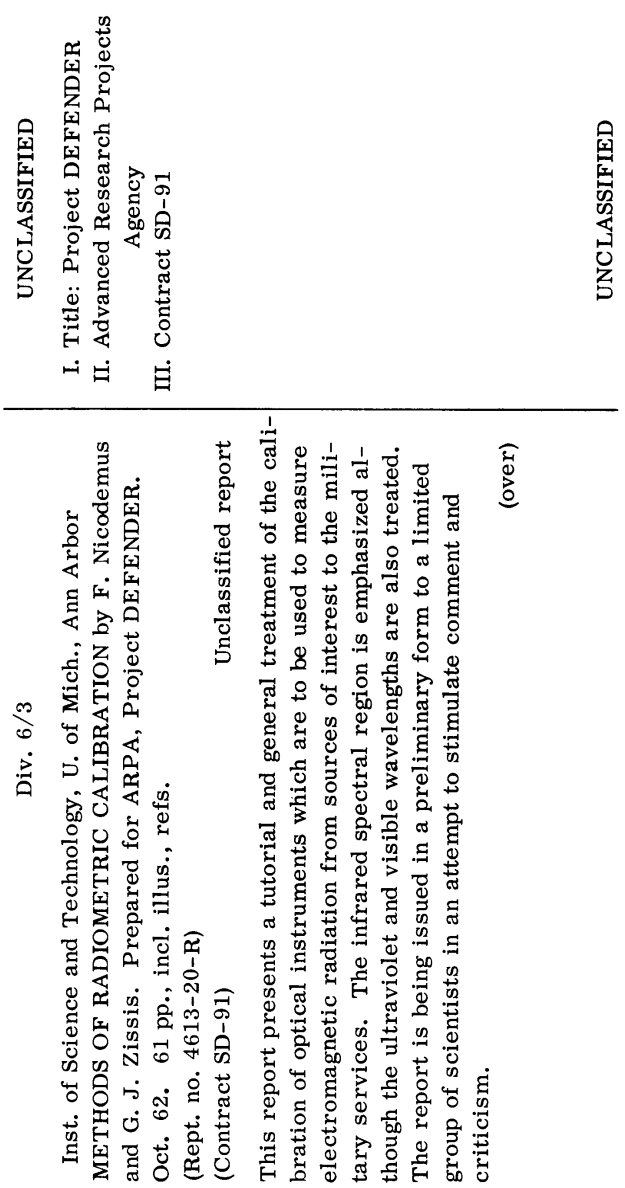

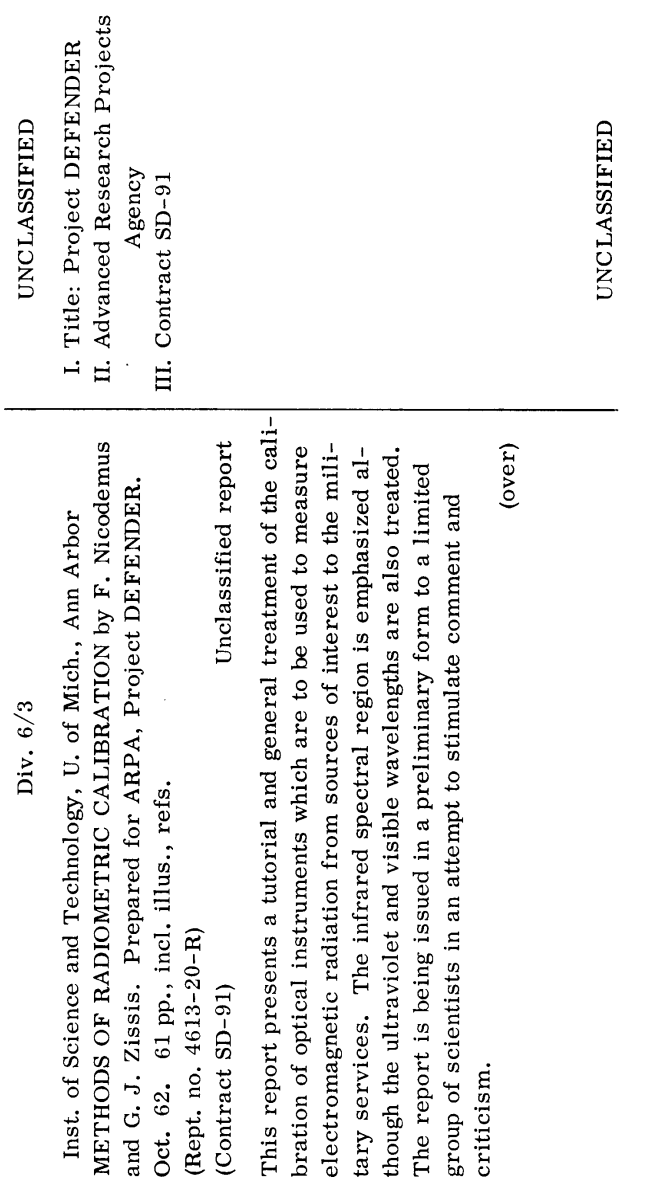

$+$
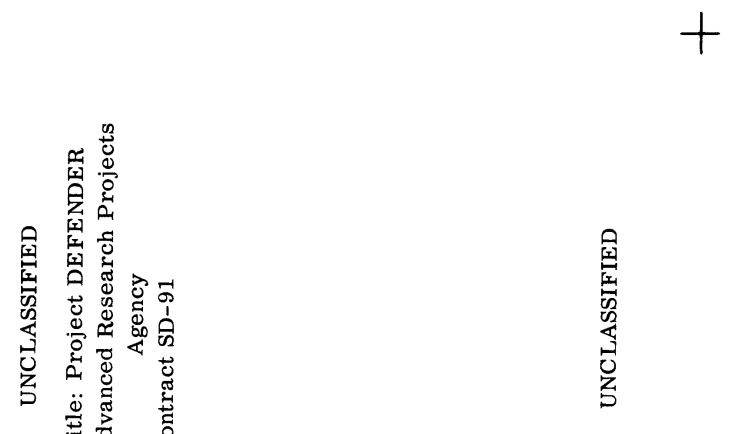

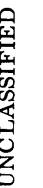

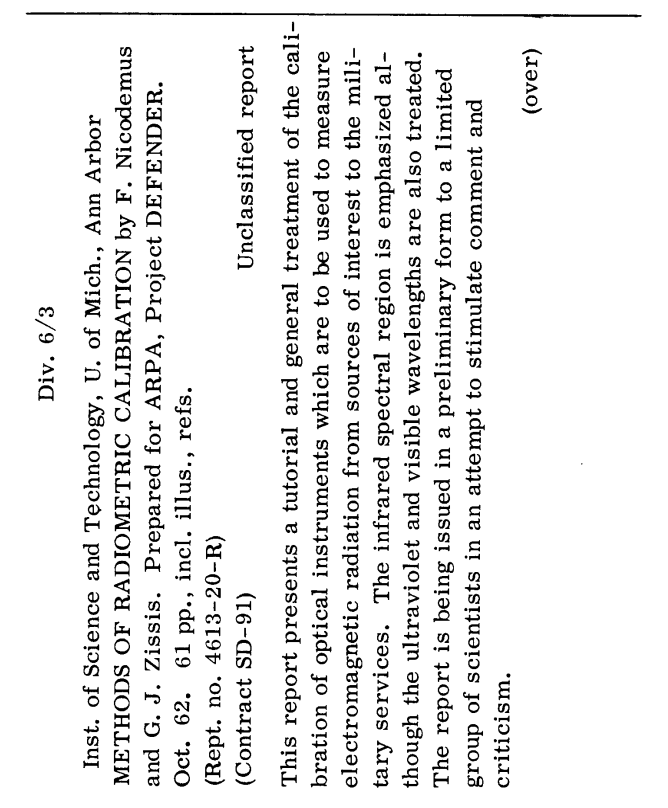



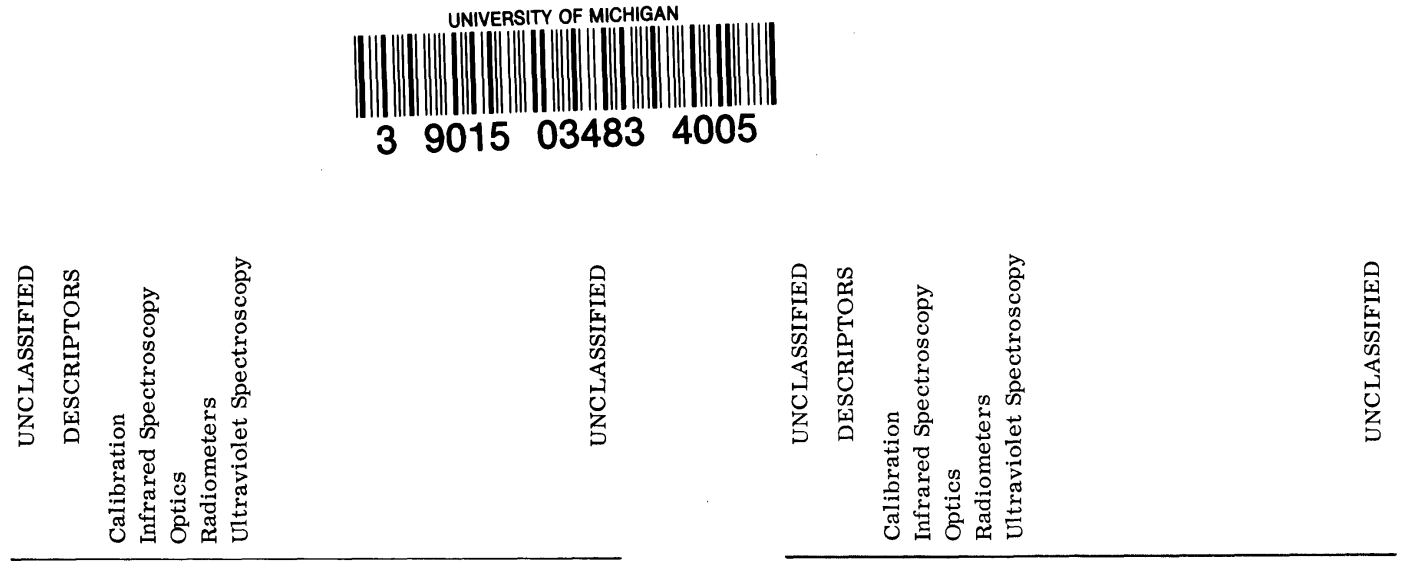

$+$
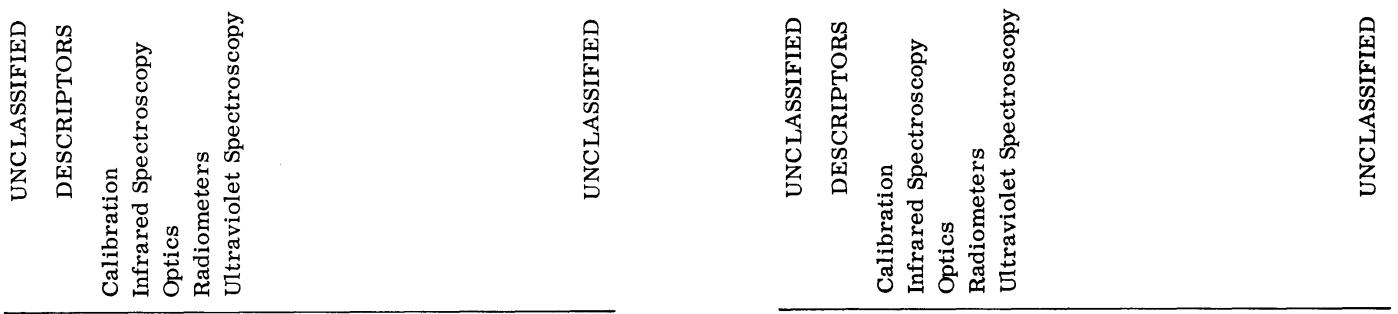